\title{
Assay Report
}

\section{Protease Inhibitor Assays}

Enzymatic Study of Three Compounds from NCATS/NIH 


\section{NIH_Protease_200603}

\section{Protease Inhibitor Assays}

Study Sponsor:

Attention:

Address:

Study Director:

Testing Facility:

Study Period:

Report Version:

Report Date:
NCATS/NIH

Jonathan Shrimp

NCATS/NIH

9800 Medical Center Drive, Rm 1034B

Rockville, MD 20850

Henry Zhu, Ph.D.

BPS Bioscience Inc.

6042 Cornerstone Court West, Ste. B

San Diego, CA 92121

USA

June 3, 2020 


\section{Study Director}

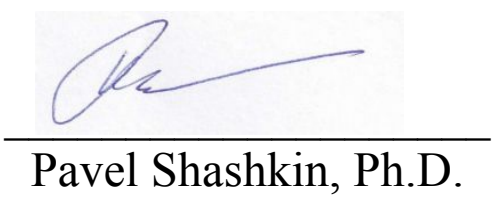

Director

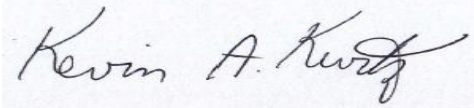

Kevin Kurtz

Sr. Scientist II.

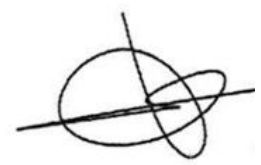

Ivan Caballero

Sr. Scientist II.

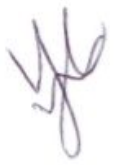

Victoria Castillo

Research Associate II.

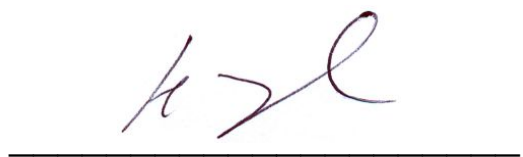

Henry Zhu, Ph.D.

President

06-03-2020

\section{Date}




\section{CONTENTS}

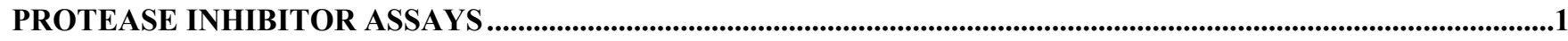

ENZYMATIC STUDY OF THREE COMPOUNDS FROM NCATS/NIH ..................................................................

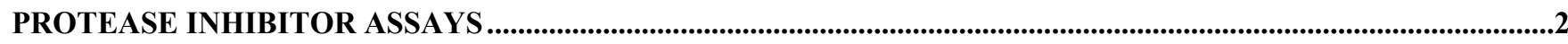

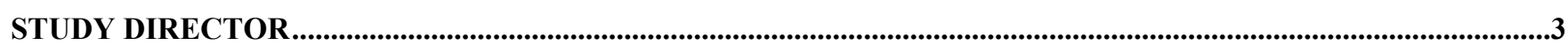

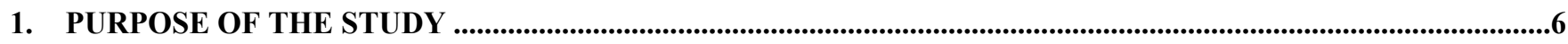

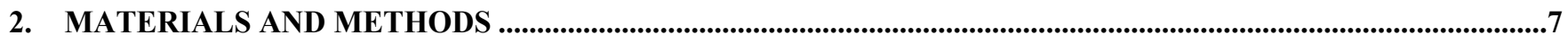

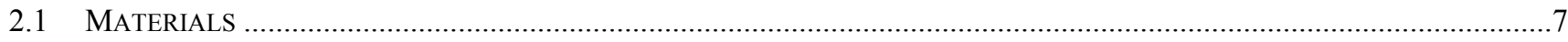

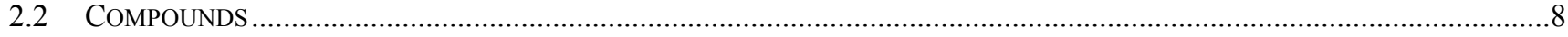

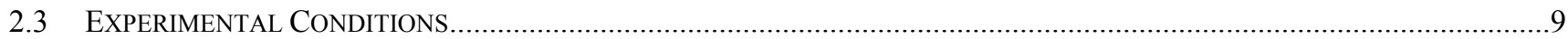

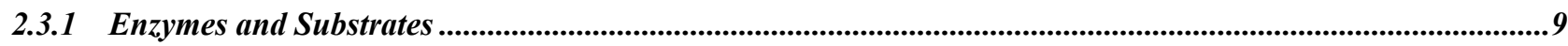

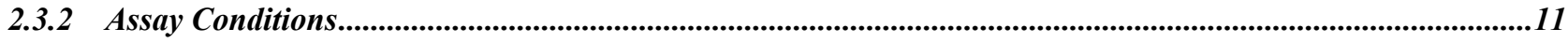

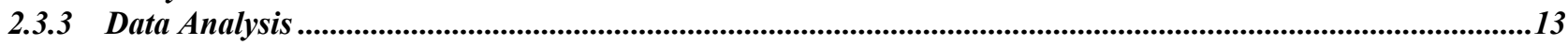

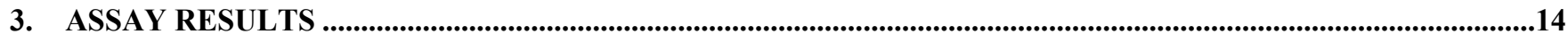

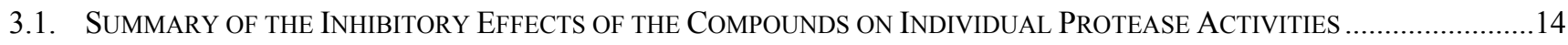

3.2. RESULTS OF THE EFFECTS OF THE COMPOUNDS ON INDIVIDUAL PROTEASE ACTIVITY ……......................................16

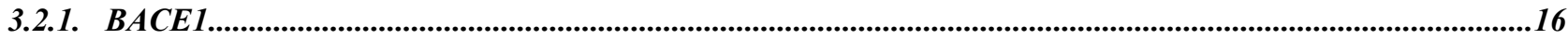

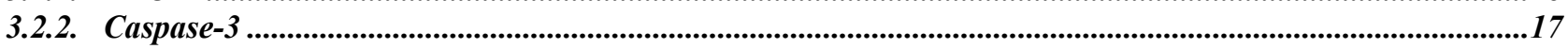

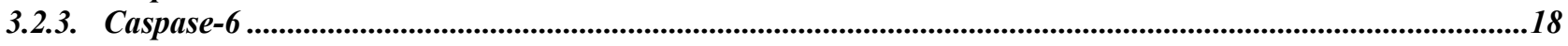

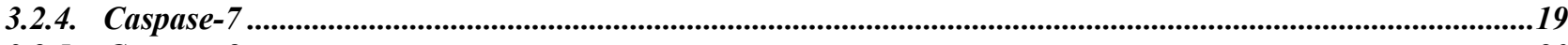

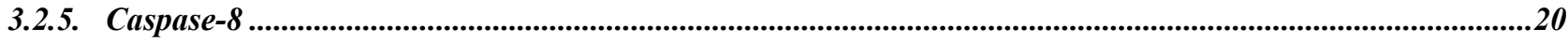

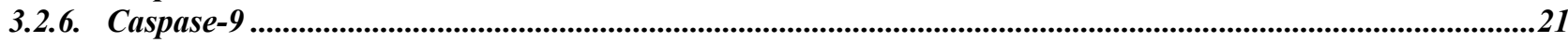

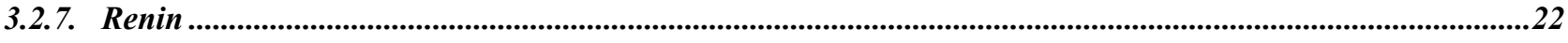

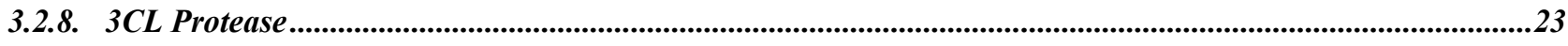

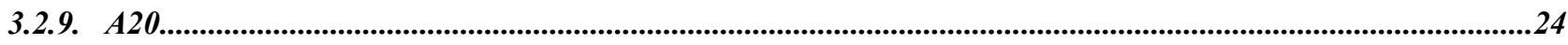

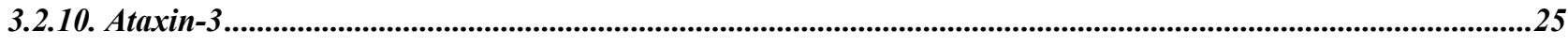

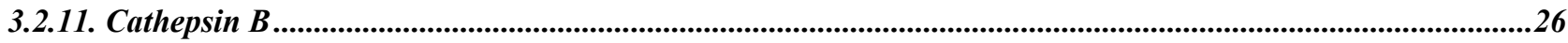

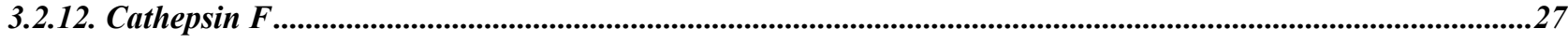

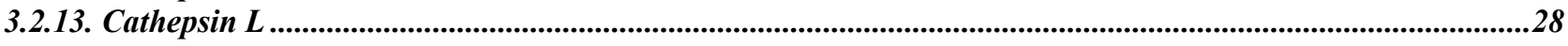

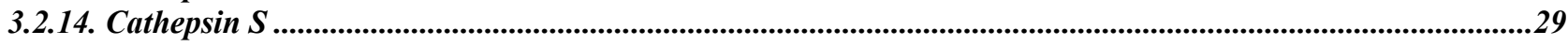

3.2.15. Cathepsin $V$............................................................................................................................................30

3.2.16. $\mathrm{MALT1}$........................................................................................................................................31

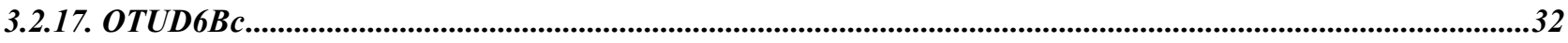

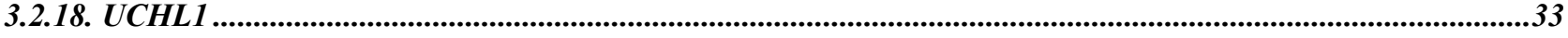

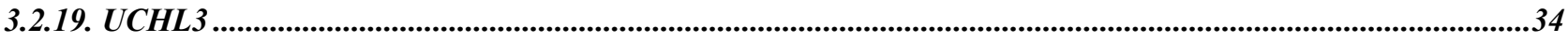

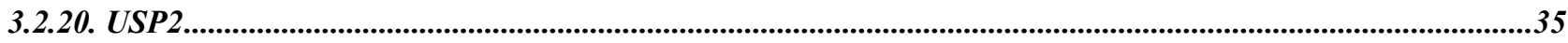

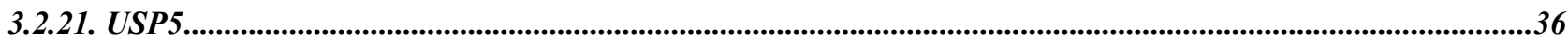

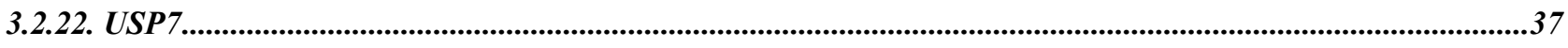

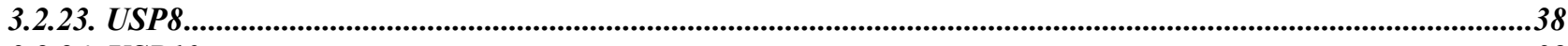

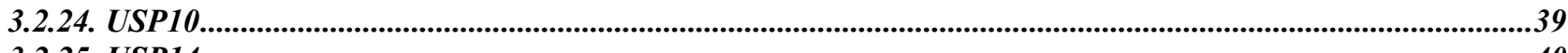

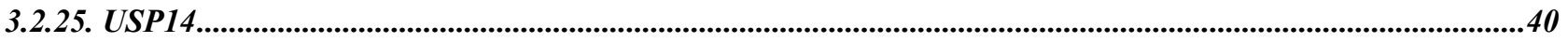




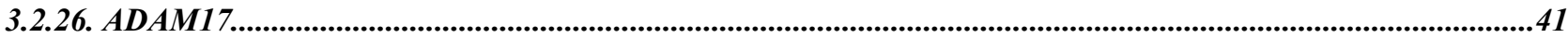

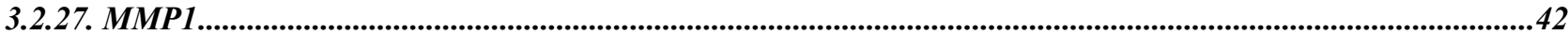

3.2.28. MMP2

3.2.29. ММР3

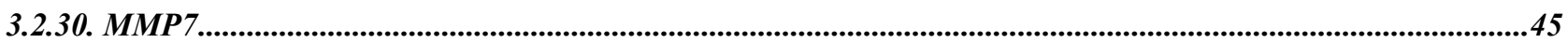

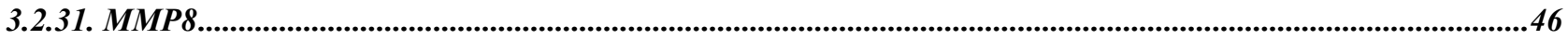

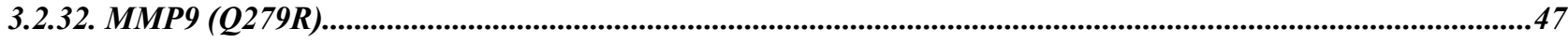

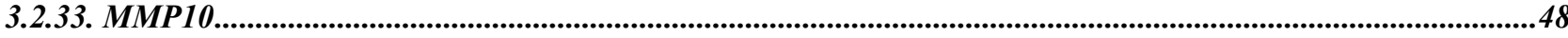

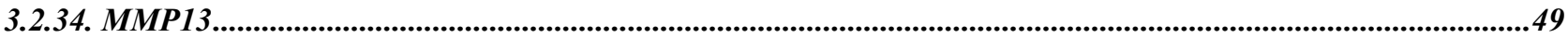

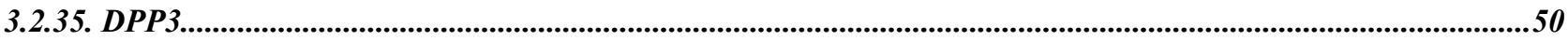

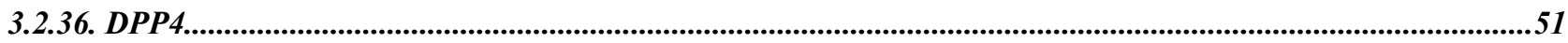

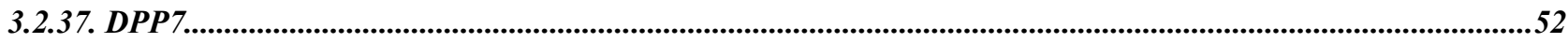

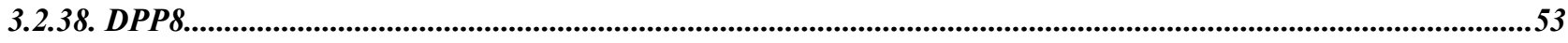

3.2.39. DPP9

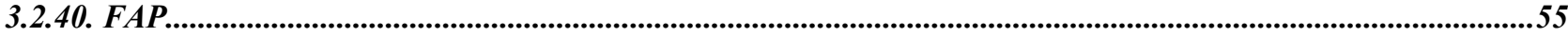

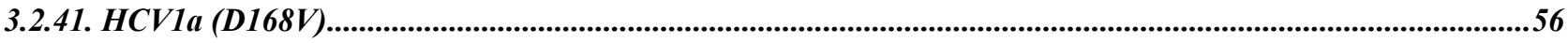

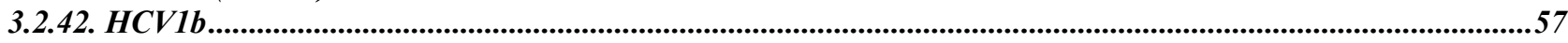

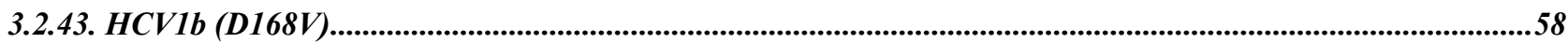

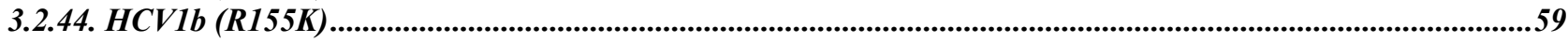

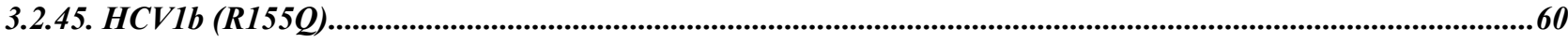

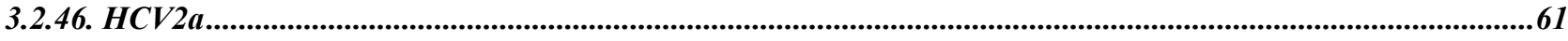

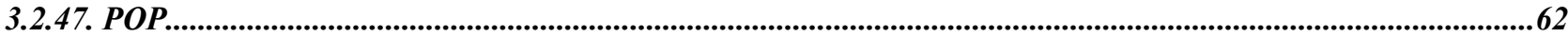

3.2.48. $А$ РC

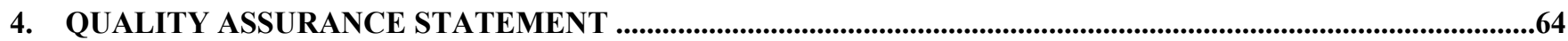




\section{1..Purpose of the Study}

The purpose of the study is to determine the effects of three compounds from NCATS/NIH on the enzymatic activities of recombinant human BACE1, Caspase-3, Caspase-6, Caspase-7, Caspase-8, Caspase-9, Renin, 3CL, A20, Ataxin-3, Cathepsin B, Cathepsin F, Cathepsin L, Cathepsin S, Cathepsin V, MALT1, OTUD6Bc, UCHL1, UCHL3, USP2, USP5, USP7, USP8, USP10, USP14, ADAM17, MMP1, MMP2, MMP3, MMP7, MMP8, MMP9 (Q279R), MMP10, MMP13, DPP3, DPP4, DPP7, DPP8, DPP9, FAP, HCV1a (D168V), HCV1b, HCV1b (D168V), HCV1b (R155K), HCV1b (R155Q), HCV2a, POP, and APC using an in vitro enzymatic assay. 


\section{Materials and Methods}

\subsection{Materials}

Verubecestat is purchased from SelleckChem (catalog number S8173).

Z-Ac-DNLD-CHO is purchased from Millipore (catalog number 218832).

Ac-IETD-CHO is purchased from Millipore (catalog number 218773).

Aliskiren hydrochloride is from BPS Bioscience (BPS number 27303).

GC376 is purchased from Aobious (catalog number AOB36447).

Ubiquitin-Aldehyde is purchased from Boston Biochem (catalog number U201).

E-64 Santa is purchased from Cruz Biotechnology (catalog number 66701-25-5).

Cystatin C is purchased from Enzo Life science (catalog number BML-SE479).

Z-VRPR-FMK is purchased from Tocris (Catalog \#4645).

TAPI-2 is purchased from Cayman Chemical (catalog number 14695).

NNGH is purchased from Sigma Aldrich (catalog number SML0584).

Batimastat is purchased from Cayman Chemical (catalog number 14742).

Spinorphin is purchased from Tocris (catalog number 2931).

Sitagliptin from BPS Bioscience (BPS number 27106).

KR62436 is purchased from Sigma-Aldrich (catalog number K4264).

SP-13786 is purchased from MedChemExpress (catalog number HY-100684).

Denoprevir is purchased from Selleckchem (catalog number S1183).

Z-Pro-Pro-CHO is purchased from Enzo Life science (catalog number BML-PI112).

Dabigatran is purchased from Selleckchem (catalog number S2196).

Z-LEHD-FMK is purchased from Selleckchem (catalog number S7313).

Boc-VPR-AMC Fluorogenic Peptide Substrate is purchased from R \& D Systems (catalog number ES011).

BACE substrate is purchased from R \& D Systems (catalog number ES004).

Caspase 3 substrate is purchased from Anaspec (catalog number 25273-5).

Ac-VEID-AFC is purchased from Enzo Lifesciences (catalog number ALX-260-111).

Ac-IETD-AFC is purchased from Enzo Lifesciences (catalog number ALX-260-110).

Ac-LEHD-AFC is purchased from Enzo Lifesciences (catalog number ALX-260-116).

Z-Leu-Arg-AMC is purchased from Enzo Lifesciences (catalog number BML-P229-

0010).

Ubiquitin-AMC is purchased from Boston Biochem (catalog number U550).

Z-Arg-Arg-AMC is purchased from BACHEM (catalog number 4004789.0050).

Fluorogenic DPP substrate 1 (Ala-Pro-AMC) is from BPS Bioscience (BPS number 80305).

Ac-LRSR-AMC is purchased from Peptides International (catalog number MCA-3952PI).

390 MMP FRET Substrate I is purchased from Anaspec (catalog number 27077).

Renin assay kit is from BPS Bioscience (BPS number 80211). 
HCV Protease FRET Substrate (RET S1) is purchased from Anaspec (catalog number AS-22991).

\subsection{Compounds}

The test compound is supplied by NCATS/NIH.

\begin{tabular}{|c|c|c|c|c|c|}
\hline Compound I.D. & $\begin{array}{l}\text { Compound } \\
\text { Supplied }\end{array}$ & $\begin{array}{c}\text { Stock } \\
\text { Concentration }\end{array}$ & $\begin{array}{c}\text { Dissolving } \\
\text { Solvent }\end{array}$ & Test Range $(\mu \mathrm{M})$ & $\begin{array}{l}\text { Intermediate } \\
\text { Dilution }\end{array}$ \\
\hline $\begin{array}{c}\text { NCGC00167526 } \\
\text { (Camostat) }\end{array}$ & Solution & $10 \mathrm{mM}$ & DMSO & 10 & $\begin{array}{c}10 \% \text { DMSO in } \\
\text { Assay Buffer }\end{array}$ \\
\hline $\begin{array}{c}\text { NCGC00160398 } \\
\text { (Nafamostat) }\end{array}$ & Solution & $10 \mathrm{mM}$ & DMSO & 10 & $\begin{array}{c}10 \% \text { DMSO in } \\
\text { Assay Buffer }\end{array}$ \\
\hline $\begin{array}{c}\text { NCGC00025297 } \\
\text { (Gabexate) }\end{array}$ & Solution & $10 \mathrm{mM}$ & DMSO & 10 & $\begin{array}{c}10 \% \text { DMSO in } \\
\text { Assay Buffer }\end{array}$ \\
\hline Verubecestat* & Solid & $10 \mathrm{mM}$ & DMSO & $0.3,0.03,0.003$ & $\begin{array}{c}10 \% \text { DMSO in } \\
\text { Assay Buffer }\end{array}$ \\
\hline Z-Ac-DNLD-CHO* & Solid & $10 \mathrm{mM}$ & DMSO & $\begin{array}{c}0.5,0.1,0.05 \\
0.01,0.001,0.005 \\
\end{array}$ & $\begin{array}{c}10 \% \text { DMSO in } \\
\text { Assay Buffer }\end{array}$ \\
\hline Ac-IETD-CHO* & Solid & $10 \mathrm{mM}$ & DMSO & $\begin{array}{c}0.5,0.1,0.05 \\
0.01,0.001,0.005 \\
\end{array}$ & $\begin{array}{c}10 \% \text { DMSO in } \\
\text { Assay Buffer }\end{array}$ \\
\hline Aliskiren* & Solid & $10 \mathrm{mM}$ & DMSO & $\begin{array}{c}0.01,0.001 \\
0.0001 \\
\end{array}$ & $\begin{array}{c}10 \% \text { DMSO in } \\
\text { Assay Buffer }\end{array}$ \\
\hline GC376* & Solid & $10 \mathrm{mM}$ & DMSO & $5,0.5,0.05$ & $\begin{array}{c}10 \% \text { DMSO in } \\
\text { Assay Buffer }\end{array}$ \\
\hline Ubiquitin-Aldehyde* & Solution & $160 \mu \mathrm{M}$ & Buffer & $100-0.0001$ & $\begin{array}{c}10 \% \text { DMSO in } \\
\text { Assay Buffer }\end{array}$ \\
\hline E-64* & Solid & $10 \mathrm{mM}$ & DMSO & $0.5,0.05,0.005$ & $\begin{array}{c}10 \% \text { DMSO in } \\
\text { Assay Buffer }\end{array}$ \\
\hline Cystatin $\mathrm{C}^{*}$ & Solid & $10 \mathrm{mM}$ & DMSO & $100,10,1$ & $\begin{array}{c}10 \% \text { DMSO in } \\
\text { Assay Buffer }\end{array}$ \\
\hline Z-VRPR-FMK* & Solid & $10 \mathrm{mM}$ & DMSO & $0.1,0.01,0.001$ & $\begin{array}{c}10 \% \text { DMSO in } \\
\text { Assay Buffer }\end{array}$ \\
\hline TAPI-2* & Solid & $10 \mathrm{mM}$ & DMSO & $10,1,0.1$ & $\begin{array}{c}10 \% \text { DMSO in } \\
\text { Assay Buffer }\end{array}$ \\
\hline $\mathrm{NNGH}^{*}$ & Solid & $10 \mathrm{mM}$ & DMSO & $\begin{array}{c}1,0.1,0.01 \\
0.001\end{array}$ & $\begin{array}{c}10 \% \text { DMSO in } \\
\text { Assay Buffer }\end{array}$ \\
\hline Batimastat* & Solid & $10 \mathrm{mM}$ & DMSO & $\begin{array}{c}0.01,0.001, \\
0.0001\end{array}$ & $\begin{array}{c}10 \% \text { DMSO in } \\
\text { Assay Buffer }\end{array}$ \\
\hline Spinorphin* & Solid & $10 \mathrm{mM}$ & DMSO & $10,0.1,0.01$ & $\begin{array}{c}10 \% \text { DMSO in } \\
\text { Assay Buffer }\end{array}$ \\
\hline
\end{tabular}




\begin{tabular}{|c|c|c|c|c|c|}
\hline Sitagliptin* & Solid & $10 \mathrm{mM}$ & DMSO & $0.1,0.01,0.001$ & $\begin{array}{c}10 \% \text { DMSO in } \\
\text { Assay Buffer }\end{array}$ \\
\hline KR62436* & Solid & $10 \mathrm{mM}$ & DMSO & $100,10,1,0.1$ & $\begin{array}{c}10 \% \text { DMSO in } \\
\text { Assay Buffer }\end{array}$ \\
\hline SP-13786* & Solid & $10 \mathrm{mM}$ & DMSO & $0.1,0.01,0.001$ & $\begin{array}{c}10 \% \text { DMSO in } \\
\text { Assay Buffer }\end{array}$ \\
\hline Denoprevir* & Solid & $10 \mathrm{mM}$ & DMSO & $\begin{array}{c}1,0.1,0.01, \\
0.001,0.0001\end{array}$ & $\begin{array}{c}10 \% \text { DMSO in } \\
\text { Assay Buffer }\end{array}$ \\
\hline Z-Pro-Pro-CHO* & Solid & $10 \mathrm{mM}$ & DMSO & $0.1,0.01,0.001$ & $\begin{array}{c}10 \% \text { DMSO in } \\
\text { Assay Buffer }\end{array}$ \\
\hline Dabigatran* & Solution & $10 \mathrm{mM}$ & DMSO & $20,2,0.2$ & $\begin{array}{c}10 \% \text { DMSO in } \\
\text { Assay Buffer }\end{array}$ \\
\hline Z-LEHD-FMK* & Solid & $10 \mathrm{mM}$ & DMSO & $10,1,0.1$ & $\begin{array}{c}10 \% \text { DMSO in } \\
\text { Assay Buffer }\end{array}$ \\
\hline
\end{tabular}

*Reference compounds.

\subsection{Experimental Conditions}

\subsubsection{Enzymes and Substrates}

\begin{tabular}{|c|c|c|c|c|}
\hline Assay & Catalog \# & Enzyme Lot \# & $\begin{array}{c}\text { Enzyme Used } \\
(\mathrm{ng}) / \text { Reaction }\end{array}$ & Substrate \\
\hline BACE1 & 71657 & 170307 & 100 & $7.5 \mu \mathrm{M}$ BACE1 Substrate \\
\hline Caspase-3 & 80500 & $130226-\mathrm{G} 1$ & 1 & $1 \mu \mathrm{M}$ Caspase 3 Substrate \\
\hline Caspase-6 & 80113 & $130222-2$ & 15 & $1 \mu \mathrm{M}$ Ac-VEID-AFC \\
\hline Caspase-7 & 70000 & 130116 & 3 & $1 \mu \mathrm{M}$ Caspase 3 Substrate \\
\hline Caspase-8 & 80114 & $130215-2$ & 5 & $1 \mu \mathrm{M}$ Ac-LEHD-AFC \\
\hline Caspase-9 & 80115 & 190306 & 2000 & $1 \mu \mathrm{M} \mathrm{Ac-LEHD-AFC}$ \\
\hline Renin & 80200 & 161221 & 4 & $5 \mu \mathrm{M}$ Renin Substrate \\
\hline 3CL Protease & 100707 & $200416-1$ & 150 & $50 \mu \mathrm{M}$ 3CL Protease Substrate \\
\hline A20 & 80408 & 171109 & 100 & $100 \mathrm{nM}$ Ub-AMC \\
\hline Ataxin3 & 80399 & 180817 & 2000 & 100 nM Ub-AMC \\
\hline Cathepsin B & 80001 & 161019 & 0.4 & $5 \mu \mathrm{M}$ Z-Leu-Arg-AMC \\
\hline Cathepsin F & 80003 & 160921 & 100 & $5 \mu \mathrm{M}$ Z-Leu-Arg-AMC \\
\hline Cathepsin L & 80005 & $170620-1$ & 0.4 & $5 \mu \mathrm{M}$ Z-Leu-Arg-AMC \\
\hline Cathepsin S & 80008 & 170214 & 20 & $5 \mu \mathrm{M}$ Z-Leu-Arg-AMC \\
\hline Cathepsin V & 80009 & 160823 & 2 & $5 \mu \mathrm{M}$ Z-Leu-Arg-AMC \\
\hline MALT1 & 100360 & $190722-1$ & 150 & $10 \mu \mathrm{M}$ Ac-LRSR-AMC \\
\hline OTUD6B & 80407 & $170926-1$ & 400 & $100 \mathrm{nM}$ Ub-AMC \\
\hline UCHL1 & 80351 & 100826 & 0.5 & $100 \mathrm{nM}$ Ub-AMC \\
\hline UCHL3 & 80353 & $190212-1$ & 0.01 & $100 \mathrm{nM}$ Ub-AMC \\
\hline
\end{tabular}


6042 Cornerstone Court West, Suite B

San Diego, CA 92121

Tel: 1.858 .829 .3082

Fax: 1.858.481.8694

Email: info@bpsbioscience.com

\begin{tabular}{|c|c|c|c|c|}
\hline USP2 & 80352 & 141212 & 5 & $100 \mathrm{nM} \mathrm{Ub-AMC}$ \\
\hline USP5 & 80355 & 120213 & 20 & $100 \mathrm{nM} \mathrm{Ub-AMC}$ \\
\hline USP7 & 80395 & $141020-\mathrm{H} 2$ & 5 & $100 \mathrm{nM} \mathrm{Ub-AMC}$ \\
\hline USP8 & 80358 & 140521-A & 40 & $100 \mathrm{nM} \mathrm{Ub-AMC}$ \\
\hline USP10 & 80360 & $140501-1$ & 200 & $100 \mathrm{nM} \mathrm{Ub}-\mathrm{AMC}$ \\
\hline USP14 & 80364 & 120227 & 20 & $100 \mathrm{nM} \mathrm{Ub-AMC}$ \\
\hline ADAM17 & - & 200529 & 20 & $1 \mu \mathrm{M}$ ADAM17 Substrate \\
\hline MMP1 & 80214 & 130730 & 10 & $\begin{array}{c}10 \mu \mathrm{M} \\
\text { 390 MMP FRET Substrate I }\end{array}$ \\
\hline MMP2 & 80213 & 160720 & 4 & $\begin{array}{c}2 \mu \mathrm{M} \\
390 \text { MMP FRET Substrate I }\end{array}$ \\
\hline MMP3 & 11346 & 130730 & 100 & $\begin{array}{c}2 \mu \mathrm{M} \\
390 \text { MMP FRET Substrate I }\end{array}$ \\
\hline MMP7 & - & - & 10 & $\begin{array}{c}2 \mu \mathrm{M} \\
\text { 390 MMP FRET Substrate I }\end{array}$ \\
\hline MMP8 & 100552 & 191217-1 & 4 & $\begin{array}{c}1 \mu \mathrm{M} \\
390 \text { MMP FRET Substrate I }\end{array}$ \\
\hline $\begin{array}{l}\text { MMP9 } \\
\text { (Q279R) }\end{array}$ & 80215 & 130730 & 2 & $\begin{array}{c}1 \mu \mathrm{M} \\
390 \text { MMP FRET Substrate I }\end{array}$ \\
\hline MMP10 & 100565 & 200115 & 100 & $\begin{array}{c}2 \mu \mathrm{M} \\
390 \text { MMP FRET Substrate I }\end{array}$ \\
\hline MMP13 & 11345 & 140219 & 100 & $\begin{array}{c}2 \mu \mathrm{M} \\
390 \text { MMP FRET Substrate I }\end{array}$ \\
\hline DPP3 & 80030 & 191211 & 10 & $\begin{array}{c}5 \mu \mathrm{M} \text { DPP Substrate } 2 \\
\text { (Arg-Arg-AMC) }\end{array}$ \\
\hline DPP4 & 80040 & 100707 & 0.2 & $\begin{array}{c}5 \mu \mathrm{M} \text { DPP Substrate } 1 \\
\text { (Ala-Pro-AMC) }\end{array}$ \\
\hline DPP7 & 80070 & $111207-1$ & 10 & $\begin{array}{c}5 \mu \mathrm{M} \text { DPP Substrate } 1 \\
\text { (Ala-Pro-AMC) }\end{array}$ \\
\hline DPP8 & 80080 & 190312-G2 & 10 & $\begin{array}{c}5 \mu \mathrm{M} \text { DPP Substrate } 1 \\
\text { (Ala-Pro-AMC) }\end{array}$ \\
\hline DPP9 & 80090 & 2000 & 2.5 & $\begin{array}{c}5 \mu \mathrm{M} \text { DPP Substrate } 1 \\
\text { (Ala-Pro-AMC) }\end{array}$ \\
\hline FAP & 80100 & 190620 & 250 & $\begin{array}{c}5 \mu \mathrm{M} \text { DPP Substrate } 1 \\
\text { (Ala-Pro-AMC) }\end{array}$ \\
\hline $\begin{array}{l}\text { HCV1a } \\
\text { (D168V) }\end{array}$ & 80103 & 130920-G2 & 50 & $5 \mu \mathrm{M}$ HCV Substrate \\
\hline HCV1b & 80107 & 120817-GC & 20 & $5 \mu \mathrm{M}$ HCV Substrate \\
\hline $\begin{array}{l}\text { HCV1b } \\
(\mathrm{D} 168 \mathrm{~V})\end{array}$ & 80110 & 171101 & 20 & $5 \mu \mathrm{M}$ HCV Substrate \\
\hline
\end{tabular}


6042 Cornerstone Court West, Suite B

San Diego, CA 92121

Tel: 1.858 .829 .3082

Fax: 1.858 .481 .8694

Email: info@bpsbioscience.com

\begin{tabular}{|c|c|c|c|c|}
\hline $\begin{array}{c}\text { HCV1b } \\
\text { (R155K) }\end{array}$ & 80109 & 120928 & 100 & $5 \mu \mathrm{M}$ HCV Substrate \\
\hline $\begin{array}{c}\text { HCV1b } \\
\text { R155Q) }\end{array}$ & 80104 & $170118 \mathrm{C}$ & 400 & $5 \mu \mathrm{M}$ HCV Substrate \\
\hline HCV2a & 80108 & 120928 & 5 & $5 \mu \mathrm{M}$ HCV Substrate \\
\hline POP & 80105 & $160513-\mathrm{E}$ & 150 & $\begin{array}{c}5 \mu \mathrm{M} \text { DPP Substrate } 1 \\
\text { (Ala-Pro-AMC) }\end{array}$ \\
\hline APC & 70019 & $130620-\mathrm{E} 1 \mathrm{D}$ & 20 & $5 \mu \mathrm{M}$ Boc-VPR-AMC \\
\hline
\end{tabular}

\subsubsection{Assay Conditions}

A compound solution ten-fold higher than the final concentration was prepared with 10 $\%$ DMSO in assay buffer and $5 \mu \mathrm{l}$ of the dilution was added to a $50 \mu \mathrm{l}$ reaction so that the final concentration of DMSO is $1 \%$ in all of reactions. All of control samples, including background and no compound controls, also contain $1 \%$ DMSO.

For activated protein $\mathrm{C}$ assay, the enzymatic reactions were conducted in duplicate at room temperature for 30 minutes in a $50 \mu 1$ mixture containing $50 \mathrm{mM}$ Tris- $\mathrm{HCl}, \mathrm{pH}$ 8.5, $150 \mathrm{mM} \mathrm{NaCl}, 10 \mathrm{mM} \mathrm{CaCl}$, $0.01 \%$ Brij-35, $10 \mu \mathrm{M}$ Boc-VPR-AMC (see 2.3.1), activated Protein $\mathrm{C}$ enzyme (see 2.3.1) and a test compound (see 2.2). Fluorescence intensity was measured at an excitation of $380 \mathrm{~nm}$ and an emission of $460 \mathrm{~nm}$ using a Tecan Infinite M1000 microplate reader.

For BACE assay, BACE1 FRET Assay Kit (BPS catalogue number 71656) was used according to the kit manual.

For caspase assays, the enzymatic reactions were conducted in duplicate at room temperature for 30 minutes in a $50 \mu \mathrm{l}$ mixture containing $10 \mathrm{mM}$ HEPES buffer, $\mathrm{pH}$ 7.4, $10 \mathrm{mM}$ EDTA, $0.05 \%$ Chaps, $5 \mathrm{mM}$ DTT, $1 \mu \mathrm{M}$ substrate (see 2.3.1), a caspase enzyme (see 2.3.1) and a test compound (see 2.2). Fluorescence intensity was measured at an excitation of $380 \mathrm{~nm}$ and an emission of $505 \mathrm{~nm}$ using a Tecan Infinite M1000 microplate reader.

For Cathepsin assays, the enzymatic reactions were conducted in duplicate at room temperature for 30 minutes in a $100 \mu \mathrm{l}$ mixture containing $50 \mathrm{mM}$ MES buffer, $\mathrm{pH}$ 5.0, $100 \mathrm{mM} \mathrm{NaCl}, 5 \mathrm{mM}$ DTT, a Cathepsin substrate (see 2.3.1), a Cathepsin enzyme (see 2.3.1) and a test compound (see 2.2). Fluorescence intensity was measured at an 


\section{Cornerstone Court West, Suite B \\ San Diego, CA 92121 \\ Tel: 1.858 .829 .3082 \\ Fax: 1.858 .481 .8694}

Email: info@bpsbioscience.com

excitation of $360 \mathrm{~nm}$ and an emission of $460 \mathrm{~nm}$ using a Tecan Infinite M1000 microplate reader.

For deubiquitinase assays, the enzymatic reactions were conducted in duplicate at room temperature for 30 minutes in a $50 \mu \mathrm{l}$ mixture containing $50 \mathrm{mM}$ Tris- $\mathrm{HCl}, \mathrm{pH} 7.4,0.5$ mM EDTA, $0.05 \%$ Tween 20, 1 mM DTT, 100 nM Ubiquitin-AMC substrate (see 2.3.1), a deubiquitinase enzyme (see 2.3.1) and a test compound (see 2.2). Fluorescence intensity was measured at an excitation of $360 \mathrm{~nm}$ and an emission of $460 \mathrm{~nm}$ using a Tecan Infinite M1000 microplate reader.

For DPP assays, the enzymatic reactions were conducted in duplicate at room temperature for 30 minutes in a $50 \mu \mathrm{l}$ mixture containing DPP assay buffer, $5 \mu \mathrm{M}$ substrate (see 2.3.1), a DPP enzyme (see 2.3.1) and a test compound (see 2.2). Fluorescence intensity was measured at an excitation of $360 \mathrm{~nm}$ and an emission of 460 $\mathrm{nm}$ using a Tecan Infinite M1000 microplate reader.

For HCV assay, the enzymatic reactions were conducted in duplicates at room temperature for 30 minutes in a $100 \mu \mathrm{l}$ mixture containing $50 \mathrm{mM}$ Tris- $\mathrm{HCl}, \mathrm{pH} 7.4$, $150 \mathrm{mM} \mathrm{NaCl}, 10 \%$ Glycerol, $5 \mathrm{mM}$ DTT, an HCV enzyme (see 2.3.1) and a test compound (see 2.2). Fluorescence intensity was measured at an excitation of $355 \mathrm{~nm}$ and an emission of $485 \mathrm{~nm}$ using a Tecan Infinite M1000 microplate reader.

For MMP assays, the enzyme is diluted to $200 \mu \mathrm{g} / \mathrm{ml}$ in $50 \mathrm{mM}$ HEPES buffer, $\mathrm{pH} 7.4$, $10 \mathrm{mM} \mathrm{CaCl} 2,0.05 \%$ Brij-35, and $1 \mathrm{mM}$ APMA, and activated at $37^{\circ} \mathrm{C}$ for 2 hours. The enzymatic reactions were conducted in duplicate at room temperature for 30 minutes in a $50 \mu \mathrm{l}$ mixture containing $50 \mathrm{mM}$ HEPES buffer, $\mathrm{pH} 7.4,10 \mathrm{mM} \mathrm{CaCl}_{2}$, $0.05 \%$ Brij-35, an MMP substrate (see 2.3.1), an MMP enzyme (see 2.3.1) and a test compound (see 2.2). Fluorescence intensity was measured at an excitation of $328 \mathrm{~nm}$ and an emission of $393 \mathrm{~nm}$ using a Tecan Infinite M1000 microplate reader.

For Renin assay, the enzymatic reactions were conducted in duplicate at room temperature for 30 minutes in a $50 \mu 1$ mixture containing $50 \mathrm{mM}$ Tris- $\mathrm{HCl}, \mathrm{pH} 7.4,100$ $\mathrm{mM} \mathrm{NaCl}, 10 \mathrm{mM} \mathrm{MgCl} 2,0.05 \%$ Tween 20, $5 \mu \mathrm{M}$ Renin substrate (see 2.3.1), Renin enzyme, and a test compound (see 2.2). Fluorescence intensity was measured at an excitation of $490 \mathrm{~nm}$ and an emission of $520 \mathrm{~nm}$ using a Tecan Infinite M1000 microplate reader.

For MALT1 assay, the enzymatic reactions were conducted in duplicate at room temperature for 60 minutes in a 50 $\mu 1$ mixture containing $50 \mathrm{mM}$ HEPES, $\mathrm{pH} 6.8,150$ $\mathrm{mM} \mathrm{NaCl}, 1 \mathrm{M} \mathrm{Na}$ Citrate, $0.05 \%$ CHAPS, $10 \mu \mathrm{M}$ Ac-LRSR-AMC (see 2.3.1), 


\section{Cornerstone Court West, Suite B \\ San Diego, CA 92121 \\ Tel: 1.858 .829 .3082 \\ Fax: 1.858 .481 .8694}

Email: info@bpsbioscience.com

MALT1 enzyme, and a test compound (see 2.2). Fluorescence intensity was measured at an excitation of $360 \mathrm{~nm}$ and an emission of $460 \mathrm{~nm}$ using a Tecan Infinite M1000 microplate reader.

For 3CL Protease assay, $30 \mu \mathrm{l}$ of 3CL Protease, and $10 \mu \mathrm{l}$ of compound diluted in assay buffer were pre-mixed in assay well. Mixture was incubated for $30 \mathrm{~min}$ at room temperature with slow shaking. The reaction was initiated by adding $10 \mu \mathrm{l}$ of $50 \mu \mathrm{M} 3 \mathrm{CL}$ Protease substrate $(50 \mu \mathrm{M}$ final, see 2.3.1). Compounds were originally diluted in DMSO, then in 3CL Protease assay buffer. Final concentration of DMSO was 1\%. Total volume was $50 \mu \mathrm{l}$. Fluorescence was measured using a M1000 Tecan microplate reader $\left(\lambda_{\mathrm{exc}}=360 \mathrm{~nm}, \lambda_{\mathrm{em}}=460 \mathrm{~nm}\right)$ after overnight reaction. Fluorescence was also measured before the substrate addition to obtain data for the intrinsic compound fluorescence. These data were subtracted from data after overnight incubation to get net values corrected to the compound fluorescence.

\subsubsection{Data Analysis}

All of the enzyme activity assays were performed in duplicates at each concentration. The fluorescent intensity data were analyzed using the computer software, Graphpad Prism. In the absence of the compound, the fluorescent intensity $\left(\mathrm{F}_{\mathrm{t}}\right)$ in each data set was defined as $100 \%$ activity. In the absence of the enzyme, the fluorescent intensity $\left(\mathrm{F}_{\mathrm{b}}\right)$ in each data set was defined as $0 \%$ activity. The percent activity in the presence of each compound was calculated according to the following equation: $\%$ activity $=\left(F-F_{b}\right) /\left(F_{t}-F_{b}\right)$, where $F=$ the fluorescent intensity in the presence of the compound.

The values of percentage activity were plotted on a bar graph. 
6042 Cornerstone Court West, Suite B

San Diego, CA 92121

Tel: 1.858 .829 .3082

Fax: 1.858 .481 .8694

Email: info@bpsbioscience.com

\section{Assav Results}

\subsection{Summary of the Inhibitory Effects of the Compounds on Individual Protease Activities}

The percentage inhibition of the three compounds against Proteases is summarized on

Table 3.1. Compound NCGC00160398 was fluorescent for most assays and background was subtracted.

Table 3.1 Inhibitory Effects of the Compounds on Protease Activities

\begin{tabular}{|c|c|c|c|c|c|c|}
\hline \multirow[b]{2}{*}{ Enzyme } & \multicolumn{6}{|c|}{ \% Inhibition } \\
\hline & $\begin{array}{c}\text { NCGC00167526 } \\
\text { (Camostat), } 10 \\
\mu M\end{array}$ & $\begin{array}{c}\text { NCGC00160398 } \\
\text { (Nafamostat), } 10 \\
\mu M^{*}\end{array}$ & $\begin{array}{c}\text { NCGC00025297 } \\
\text { (Gabexate), } 10 \\
\mu \mathrm{M}\end{array}$ & $\begin{array}{c}\text { Reference } \\
\left(\sim 0.1 \times \mathrm{IC}_{50}\right)\end{array}$ & $\begin{array}{l}\text { Reference } \\
\left(\sim 1 \times I_{50}\right)\end{array}$ & $\begin{array}{c}\text { Reference } \\
\left(\sim 10 \times I_{50}\right)\end{array}$ \\
\hline BACE1 & 4 & 0 & 0 & 6 & 52 & 95 \\
\hline Caspase-3 & 0 & 0 & 2 & 10 & 25 & 80 \\
\hline Caspase-6 & 3 & 4 & 0 & 8 & 34 & 93 \\
\hline Caspase-7 & 0 & 1 & 1 & 8 & 43 & 89 \\
\hline Caspase-8 & 0 & 0 & 1 & 0 & 38 & 78 \\
\hline Caspase-9 & 0 & 0 & 7 & 7 & 50 & 86 \\
\hline Renin & 1 & 45 & 0 & 0 & 11 & 70 \\
\hline 3CL & 6 & 13 & 3 & 11 & 62 & 96 \\
\hline A20 & 4 & 13 & 4 & 11 & 54 & 96 \\
\hline Ataxin-3 & 3 & 0 & 3 & 30 & 67 & 96 \\
\hline Cathepsin B & 0 & 48 & 0 & 32 & 77 & 97 \\
\hline Cathepsin F & 0 & 0 & 2 & 20 & 73 & 89 \\
\hline Cathepsin L & 2 & 57 & 0 & 13 & 39 & 84 \\
\hline Cathepsin S & 3 & 8 & 4 & 18 & 81 & 97 \\
\hline Cathepsin V & 1 & 27 & 3 & 17 & 57 & 94 \\
\hline MALT1 & 48 & 49 & 46 & 9 & 56 & 97 \\
\hline OTUD6Bc & 1 & 9 & 3 & 6 & 24 & 100 \\
\hline UCHL1 & 7 & 0 & 5 & 7 & 20 & 71 \\
\hline UCHL3 & 3 & 6 & 4 & 4 & 33 & 89 \\
\hline USP2 & 1 & 0 & 2 & 5 & 55 & 96 \\
\hline USP5 & 1 & 0 & 1 & 2 & 57 & 97 \\
\hline USP7 & 0 & 0 & 0 & 12 & 56 & 97 \\
\hline
\end{tabular}


6042 Cornerstone Court West, Suite B

San Diego, CA 92121

Tel: 1.858 .829 .3082

Fax: 1.858 .481 .8694

Email: info@bpsbioscience.com

\begin{tabular}{|c|c|c|c|c|c|c|}
\hline USP8 & 0 & 5 & 0 & 11 & 35 & 85 \\
\hline USP10 & 2 & 0 & 3 & 5 & 43 & 83 \\
\hline USP14 & 0 & 0 & 1 & 19 & 72 & 100 \\
\hline ADAM17 & 6 & 5 & 7 & 0 & 13 & 73 \\
\hline MMP1 & 3 & 0 & 2 & 2 & 26 & 77 \\
\hline MMP2 & 83 & 0 & 26 & 24 & 71 & 100 \\
\hline MMP3 & 27 & 0 & 18 & 20 & 80 & 100 \\
\hline MMP7 & 2 & 5 & 3 & 4 & 31 & 94 \\
\hline MMP8 & 33 & 0 & 7 & 5 & 43 & 91 \\
\hline MMP9 (Q279R) & 8 & 11 & 13 & 27 & 74 & 96 \\
\hline MMP10 & 29 & 0 & 12 & 25 & 63 & 91 \\
\hline MMP13 & 24 & 75 & 13 & 11 & 42 & 95 \\
\hline DPP3 & 8 & 3 & 4 & 0 & 82 & 99 \\
\hline DPP4 & 17 & 18 & 21 & 13 & 53 & 93 \\
\hline DPP7 & 20 & 29 & 83 & 8 & 30 & 77 \\
\hline DPP8 & 8 & 8 & 13 & 0 & 15 & 81 \\
\hline DPP9 & 18 & 13 & 14 & 0 & 49 & 87 \\
\hline FAP & 30 & 25 & 29 & 1 & 35 & 100 \\
\hline HCV1a (D168V) & 5 & 66 & 1 & 18 & 59 & 92 \\
\hline HCV1b & 11 & 46 & 29 & 26 & 58 & 93 \\
\hline HCV1b (D168V) & 5 & 44 & 13 & 30 & 78 & 98 \\
\hline HCV1b (R155K) & 1 & 41 & 0 & 18 & 57 & 94 \\
\hline HCV1b (R155Q) & 5 & 58 & 12 & 22 & 51 & 99 \\
\hline HCV2a & 1 & 20 & 0 & 11 & 55 & 97 \\
\hline POP & 31 & 29 & 27 & 7 & 29 & 100 \\
\hline APC & 77 & 98 & 99 & 16 & 43 & 95 \\
\hline
\end{tabular}

*Compound was fluorescent for most assays. 
6042 Cornerstone Court West, Suite B

San Diego, CA 92121

Tel: 1.858 .829 .3082

Fax: 1.858 .481 .8694

Email: info@bpsbioscience.com

\subsection{Results of the Effects of the Compounds on Individual Protease Activity}

\subsubsection{BACE1}

Table 3.2.1. Data for the Effect of the Compounds on BACE1 Activity

\begin{tabular}{|c|c|c|c|c|c|c|c|}
\hline \multirow[t]{2}{*}{ Compounds } & \multicolumn{2}{|c|}{$\begin{array}{c}\text { BACE1 Activity } \\
\text { (Fluorescence count) }\end{array}$} & \multicolumn{2}{|c|}{$\begin{array}{c}\text { Background } \\
\text { (Fluorescence count) }\end{array}$} & \multicolumn{2}{|c|}{$\%$ Activity } & \multirow[t]{2}{*}{$\%$ Inhibition } \\
\hline & Repeat1 & Repeat2 & Repeat1 & Repeat2 & Repeat1 & Repeat2 & \\
\hline No Compound & 543 & 542 & 62 & 65 & 100 & 100 & 0 \\
\hline NCGC00167526, $10 \mu \mathrm{M}$ & 501 & 530 & 60 & 53 & 93 & 99 & 4 \\
\hline NCGC00160398, $10 \mu \mathrm{M}$ & 845 & 788 & 334 & 325 & 108 & 96 & 0 \\
\hline NCGC00025297, $10 \mu \mathrm{M}$ & 522 & 553 & 61 & 53 & 97 & 103 & 0 \\
\hline Verubecestat, $0.003 \mu \mathrm{M}$ & 501 & 517 & 58 & 54 & 93 & 96 & 6 \\
\hline Verubecestat, $0.03 \mu \mathrm{M}$ & 290 & 287 & 60 & 53 & 48 & 48 & 52 \\
\hline Verubecestat, $0.3 \mu \mathrm{M}$ & 86 & 84 & 59 & 53 & 5 & 5 & 95 \\
\hline Background & 67 & 65 & 62 & 63 & & & \\
\hline
\end{tabular}

BACE1 Activity

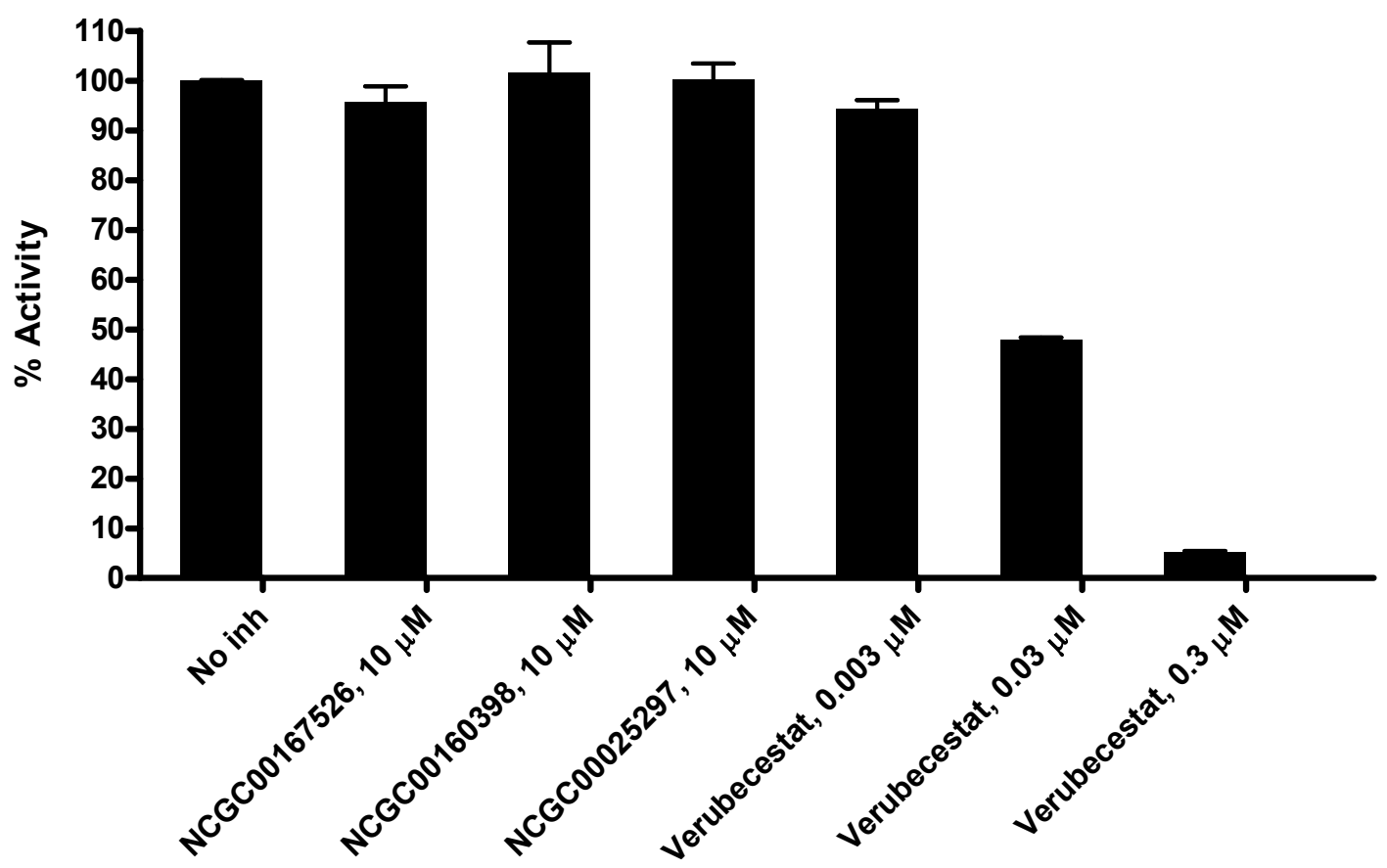


6042 Cornerstone Court West, Suite B

San Diego, CA 92121

Tel: 1.858 .829 .3082

Fax: 1.858 .481 .8694

Email: info@bpsbioscience.com

\subsubsection{Caspase-3}

Table 3.2.2. Data for the Effect of the Compounds on Caspase-3 Activity

\begin{tabular}{|c|c|c|c|c|c|c|c|}
\hline \multirow[t]{2}{*}{ Compounds } & \multicolumn{2}{|c|}{$\begin{array}{l}\text { Caspase-3 Activity } \\
\text { (Fluorescence count) }\end{array}$} & \multicolumn{2}{|c|}{$\begin{array}{c}\text { Background } \\
\text { (Fluorescence count) }\end{array}$} & \multicolumn{2}{|c|}{$\%$ Activity } & \multirow[t]{2}{*}{$\%$ Inhibition } \\
\hline & Repeat1 & Repeat2 & Repeat1 & Repeat2 & Repeat1 & Repeat2 & \\
\hline No Compound & 733 & 724 & 62 & 56 & 100 & 100 & 0 \\
\hline NCGC00167526, $10 \mu \mathrm{M}$ & 728 & 732 & 61 & 59 & 100 & 101 & 0 \\
\hline NCGC00160398, $10 \mu \mathrm{M}$ & 1304 & 1244 & 636 & 559 & 100 & 102 & 0 \\
\hline NCGC00025297, $10 \mu \mathrm{M}$ & 715 & 725 & 69 & 60 & 96 & 99 & 2 \\
\hline Ac-DNLD-CHO, $0.001 \mu \mathrm{M}$ & 677 & 664 & 62 & 70 & 92 & 89 & 10 \\
\hline Ac-DNLD-CHO, $0.01 \mu \mathrm{M}$ & 566 & 568 & 58 & 65 & 76 & 75 & 25 \\
\hline Ac-DNLD-CHO, $0.1 \mu \mathrm{M}$ & 195 & 195 & 55 & 68 & 21 & 19 & 80 \\
\hline Background & 60 & 64 & 58 & 62 & & & \\
\hline
\end{tabular}

Caspase-3 Activity

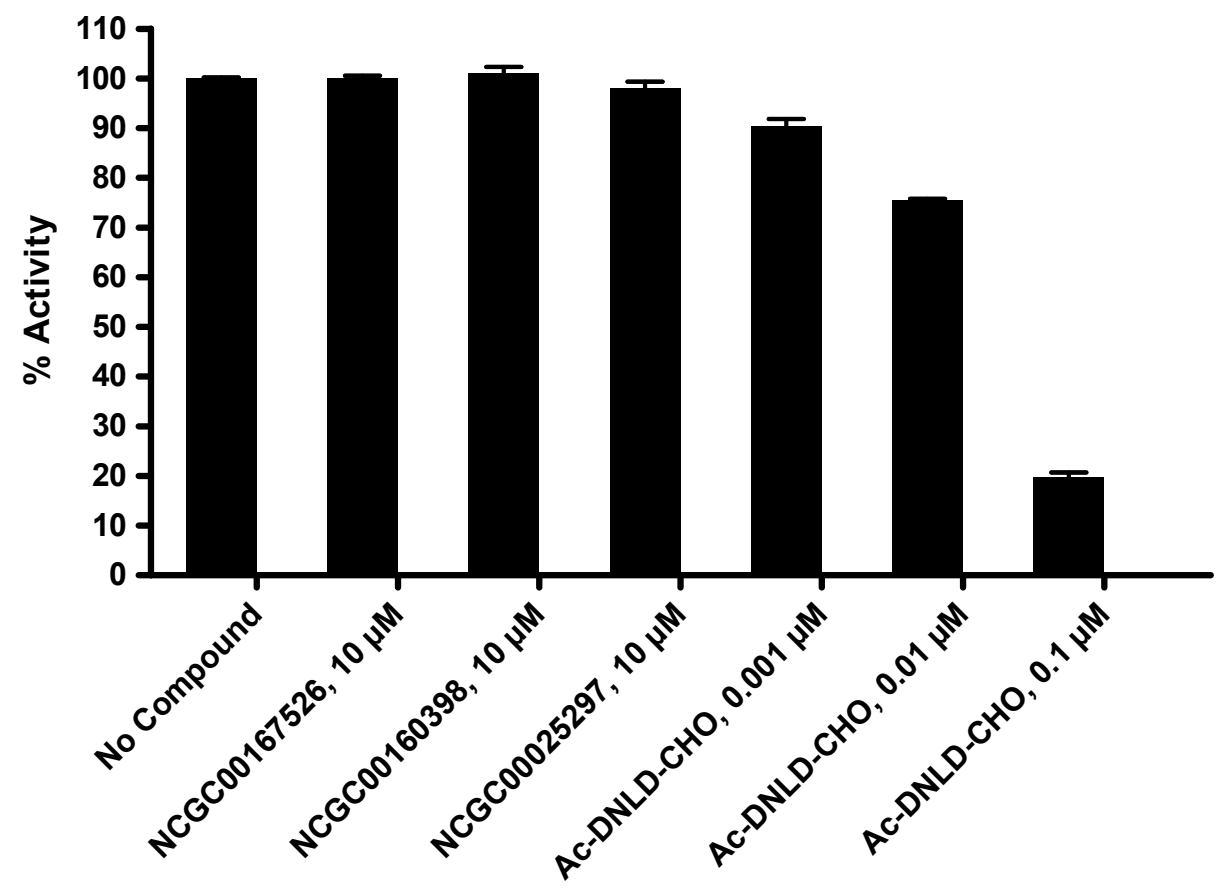




\section{Cornerstone Court West, Suite B}

San Diego, CA 92121

Tel: 1.858 .829 .3082

Fax: 1.858 .481 .8694

Email: info@bpsbioscience.com

\subsubsection{Caspase-6}

Table 3.2.3. Data for the Effect of the Compounds on Caspase-6 Activity

\begin{tabular}{|c|c|c|c|c|c|c|c|}
\hline \multirow{2}{*}{ Compounds } & \multicolumn{2}{|c|}{$\begin{array}{l}\text { Caspase-6 Activity } \\
\text { (Fluorescence count) }\end{array}$} & \multicolumn{2}{|c|}{$\begin{array}{c}\text { Background } \\
\text { (Fluorescence count) }\end{array}$} & \multicolumn{2}{|c|}{$\%$ Activity } & \multirow[t]{2}{*}{$\%$ Inhibition } \\
\hline & Repeat1 & Repeat2 & Repeat1 & Repeat2 & Repeat1 & Repeat2 & \\
\hline No Compound & 491 & 477 & 26 & 20 & 101 & 99 & 0 \\
\hline NCGC00167526, $10 \mu \mathrm{M}$ & 474 & 478 & 32 & 25 & 96 & 98 & 3 \\
\hline NCGC00160398, $10 \mu \mathrm{M}$ & 1077 & 1059 & 629 & 626 & 97 & 94 & 4 \\
\hline NCGC00025297, $10 \mu \mathrm{M}$ & 485 & 487 & 27 & 24 & 99 & 100 & 0 \\
\hline Ac-IETD-CHO, $0.05 \mu \mathrm{M}$ & 453 & 461 & 33 & 28 & 91 & 94 & 8 \\
\hline Ac-IETD-CHO, $0.5 \mu \mathrm{M}$ & 333 & 328 & 22 & 31 & 67 & 64 & 34 \\
\hline Ac-IETD-CHO, $5 \mu \mathrm{M}$ & 61 & 62 & 28 & 25 & 7 & 8 & 93 \\
\hline Background & 22 & 23 & 21 & 22 & & & \\
\hline
\end{tabular}

Caspase-6 Activity

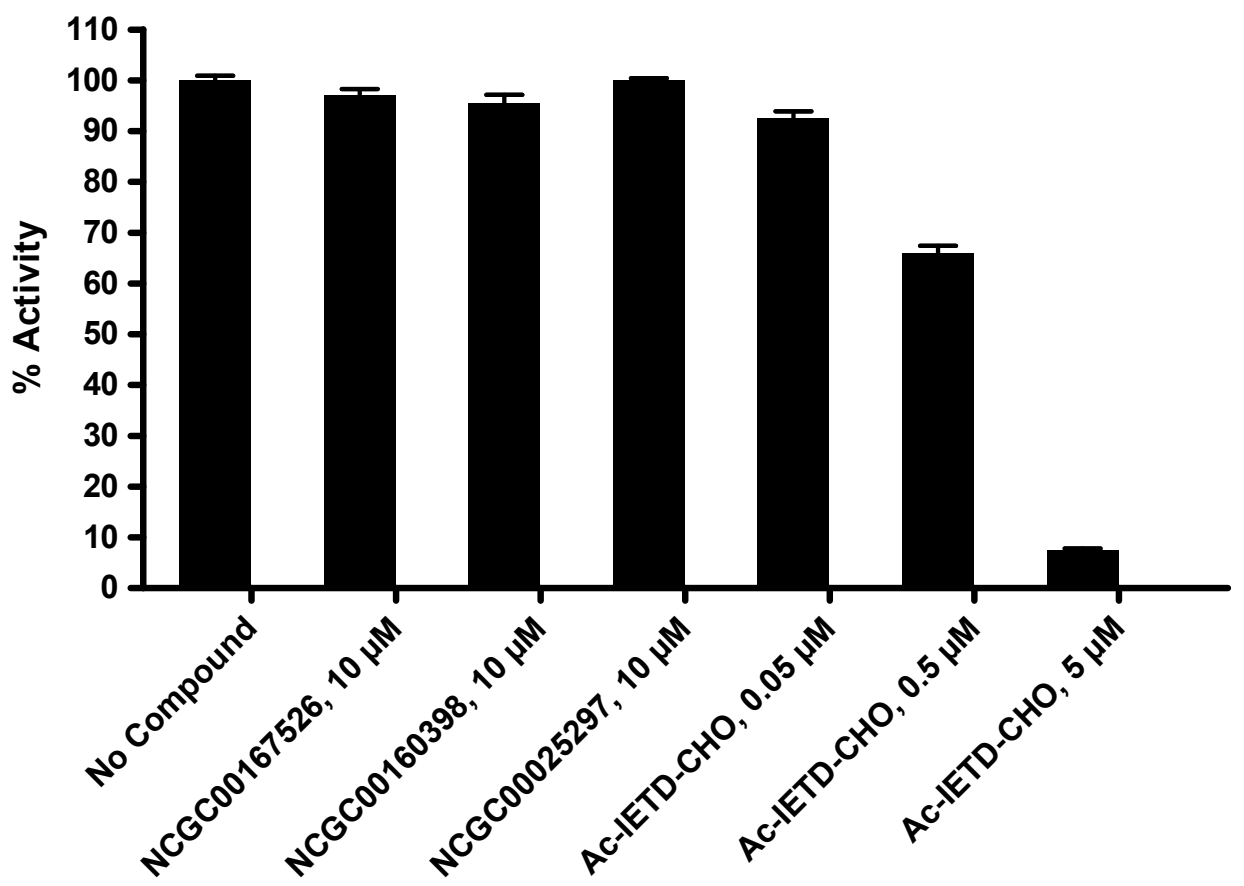


6042 Cornerstone Court West, Suite B

San Diego, CA 92121

Tel: 1.858 .829 .3082

Fax: 1.858 .481 .8694

Email: info@bpsbioscience.com

\subsubsection{Caspase-7}

Table 3.2.4. Data for the Effect of the Compounds on Caspase-7 Activity

\begin{tabular}{|c|c|c|c|c|c|c|c|}
\hline \multirow[t]{2}{*}{ Compounds } & \multicolumn{2}{|c|}{$\begin{array}{l}\text { Caspase-7 Activity } \\
\text { (Fluorescence count) }\end{array}$} & \multicolumn{2}{|c|}{$\begin{array}{c}\text { Background } \\
\text { (Fluorescence count) }\end{array}$} & \multicolumn{2}{|c|}{$\%$ Activity } & \multirow[t]{2}{*}{$\%$ Inhibition } \\
\hline & Repeat1 & Repeat2 & Repeat1 & Repeat2 & Repeat1 & Repeat2 & \\
\hline No Compound & 731 & 702 & 62 & 57 & 102 & 98 & 0 \\
\hline NCGC00167526, $10 \mu \mathrm{M}$ & 718 & 731 & 59 & 64 & 100 & 102 & 0 \\
\hline NCGC00160398, $10 \mu \mathrm{M}$ & 1251 & 1257 & 596 & 614 & 100 & 98 & 1 \\
\hline NCGC00025297, $10 \mu \mathrm{M}$ & 700 & 713 & 55 & 59 & 98 & 100 & 1 \\
\hline Ac-DNLD-CHO, $0.005 \mu \mathrm{M}$ & 676 & 659 & 58 & 63 & 94 & 91 & 8 \\
\hline Ac-DNLD-CHO, $0.05 \mu \mathrm{M}$ & 433 & 439 & 60 & 54 & 57 & 58 & 43 \\
\hline Ac-DNLD-CHO, $0.5 \mu \mathrm{M}$ & 129 & 130 & 61 & 52 & 10 & 11 & 89 \\
\hline Background & 57 & 58 & 53 & 56 & & & \\
\hline
\end{tabular}

Caspase-7 Activity

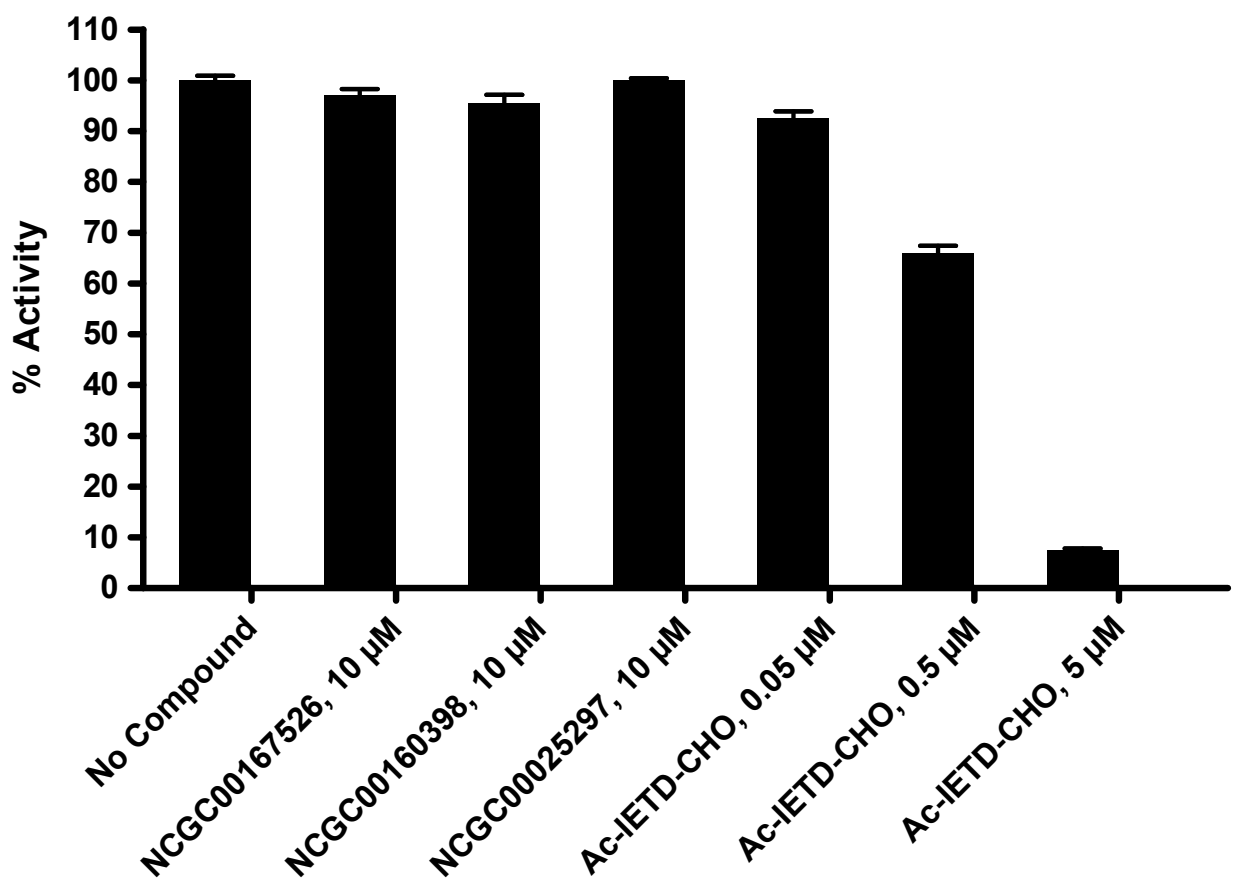


6042 Cornerstone Court West, Suite B

San Diego, CA 92121

Tel: 1.858 .829 .3082

Fax: 1.858 .481 .8694

Email: info@bpsbioscience.com

\subsubsection{Caspase-8}

Table 3.2.5. Data for the Effect of the Compounds on Caspase-8 Activity

\begin{tabular}{|c|c|c|c|c|c|c|c|}
\hline \multirow{2}{*}{ Compounds } & \multicolumn{2}{|c|}{$\begin{array}{l}\text { Caspase- } 8 \text { Activity } \\
\text { (Fluorescence count) }\end{array}$} & \multicolumn{2}{|c|}{$\begin{array}{c}\text { Background } \\
\text { (Fluorescence count) }\end{array}$} & \multicolumn{2}{|c|}{$\%$ Activity } & \multirow[t]{2}{*}{$\%$ Inhibition } \\
\hline & Repeat1 & Repeat2 & Repeat1 & Repeat2 & Repeat1 & Repeat2 & \\
\hline No Compound & 434 & 420 & 32 & 25 & 101 & 99 & 0 \\
\hline NCGC00167526, $10 \mu \mathrm{M}$ & 430 & 425 & 26 & 26 & 101 & 100 & 0 \\
\hline NCGC00160398, $10 \mu \mathrm{M}$ & 1009 & 981 & 602 & 592 & 102 & 98 & 0 \\
\hline NCGC00025297, $10 \mu \mathrm{M}$ & 425 & 420 & 28 & 30 & 100 & 98 & 1 \\
\hline Ac-IETD-CHO, $0.001 \mu \mathrm{M}$ & 417 & 428 & 25 & 23 & 98 & 102 & 0 \\
\hline Ac-IETD-CHO, $0.01 \mu \mathrm{M}$ & 271 & 277 & 29 & 24 & 60 & 63 & 38 \\
\hline Ac-IETD-CHO, $0.1 \mu \mathrm{M}$ & 120 & 123 & 29 & 33 & 22 & 22 & 78 \\
\hline Background & 32 & 31 & 30 & 28 & & & \\
\hline
\end{tabular}

Caspase-8 Activity

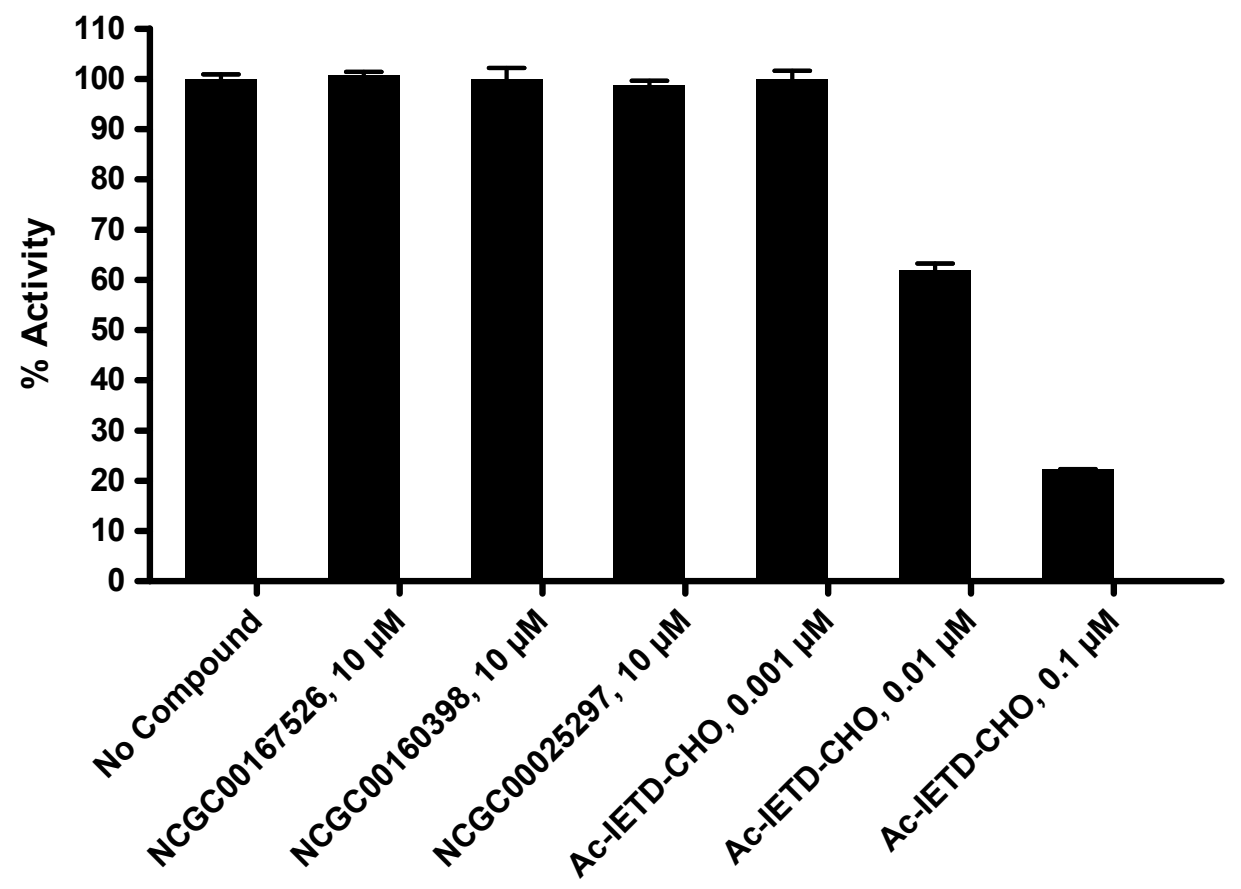




\section{Cornerstone Court West, Suite B}

San Diego, CA 92121

Tel: 1.858 .829 .3082

Fax: 1.858 .481 .8694

Email: info@bpsbioscience.com

\subsubsection{Caspase-9}

Table 3.2.6. Data for the Effect of the Compounds on Caspase-9 Activity

\begin{tabular}{|c|c|c|c|c|c|c|c|}
\hline \multirow{2}{*}{ Compounds } & \multicolumn{2}{|c|}{$\begin{array}{l}\text { Caspase-9 Activity } \\
\text { (Fluorescence count) }\end{array}$} & \multicolumn{2}{|c|}{$\begin{array}{c}\text { Background } \\
\text { (Fluorescence count) }\end{array}$} & \multicolumn{2}{|c|}{$\%$ Activity } & \multirow[t]{2}{*}{$\%$ Inhibition } \\
\hline & Repeat1 & Repeat2 & Repeat1 & Repeat2 & Repeat1 & Repeat2 & \\
\hline No Compound & 6584 & 6534 & 2048 & 1656 & 96 & 104 & 0 \\
\hline NCGC00167526, $10 \mu \mathrm{M}$ & 7008 & 7517 & 1490 & 1561 & 118 & 127 & 0 \\
\hline NCGC00160398, $10 \mu \mathrm{M}$ & 16405 & 16946 & 5338 & 5404 & 238 & 248 & 0 \\
\hline NCGC00025297, $10 \mu \mathrm{M}$ & 7544 & 6002 & 3235 & 1528 & 91 & 95 & 7 \\
\hline Z-LEHD-FMK, $0.1 \mu \mathrm{M}$ & 6160 & 5736 & 1612 & 1527 & 97 & 89 & 7 \\
\hline Z-LEHD-FMK, $1 \mu \mathrm{M}$ & 3618 & 4616 & 1555 & 1864 & 43 & 58 & 50 \\
\hline Z-LEHD-FMK, $10 \mu \mathrm{M}$ & 2372 & 2308 & 1577 & 1578 & 15 & 14 & 86 \\
\hline Background & 2115 & 2049 & 1968 & 1989 & & & \\
\hline
\end{tabular}

\section{Caspase-9 Activity}

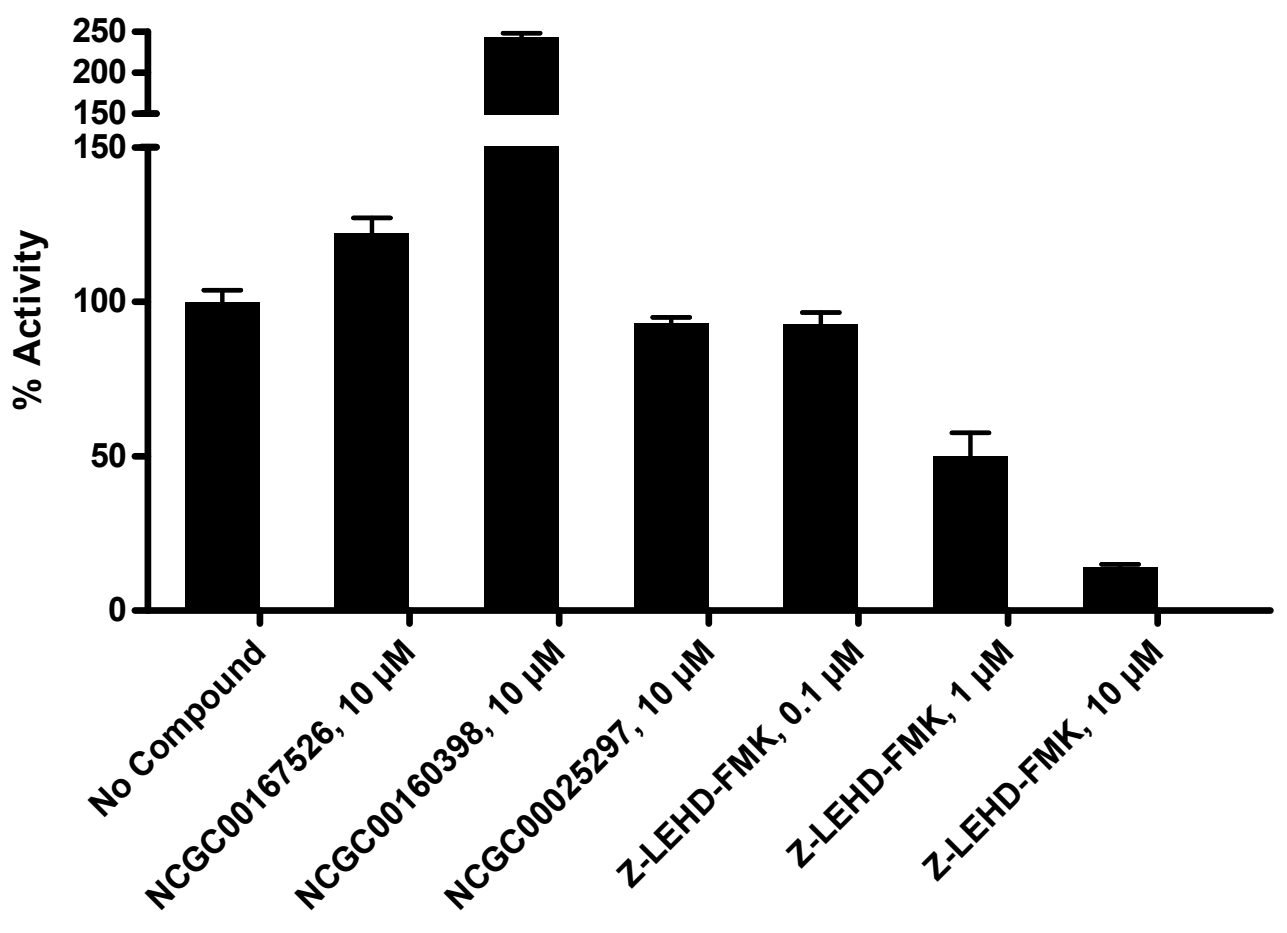


6042 Cornerstone Court West, Suite B

San Diego, CA 92121

Tel: 1.858 .829 .3082

Fax: 1.858 .481 .8694

Email: info@bpsbioscience.com

\subsubsection{Renin}

Table 3.2.7. Data for the Effect of the Compounds on Renin Activity

\begin{tabular}{|c|c|c|c|c|c|}
\hline \multirow[t]{2}{*}{ Compounds } & \multicolumn{2}{|c|}{$\begin{array}{c}\text { Renin Activity } \\
\text { (Fluorescence count) }\end{array}$} & \multicolumn{2}{|c|}{$\%$ Activity } & \multirow[t]{2}{*}{$\%$ Inhibition } \\
\hline & Repeat1 & Repeat2 & Repeat1 & Repeat2 & \\
\hline No Compound & 12253 & 12252 & 100 & 100 & 0 \\
\hline NCGC00167526, $10 \mu \mathrm{M}$ & 12115 & 12221 & 98 & 100 & 1 \\
\hline NCGC00160398, $10 \mu \mathrm{M}$ & 8395 & 8621 & 53 & 56 & 45 \\
\hline NCGC00025297, $10 \mu \mathrm{M}$ & 12390 & 12284 & 102 & 100 & 0 \\
\hline Aliskiren, $0.0001 \mu \mathrm{M}$ & 12335 & 12289 & 101 & 100 & 0 \\
\hline Aliskiren, $0.001 \mu \mathrm{M}$ & 11289 & 11463 & 88 & 90 & 11 \\
\hline Aliskiren, $0.01 \mu \mathrm{M}$ & 6377 & 6625 & 29 & 32 & 70 \\
\hline Background & 3983 & 3999 & & & \\
\hline
\end{tabular}

\section{Renin Activity}

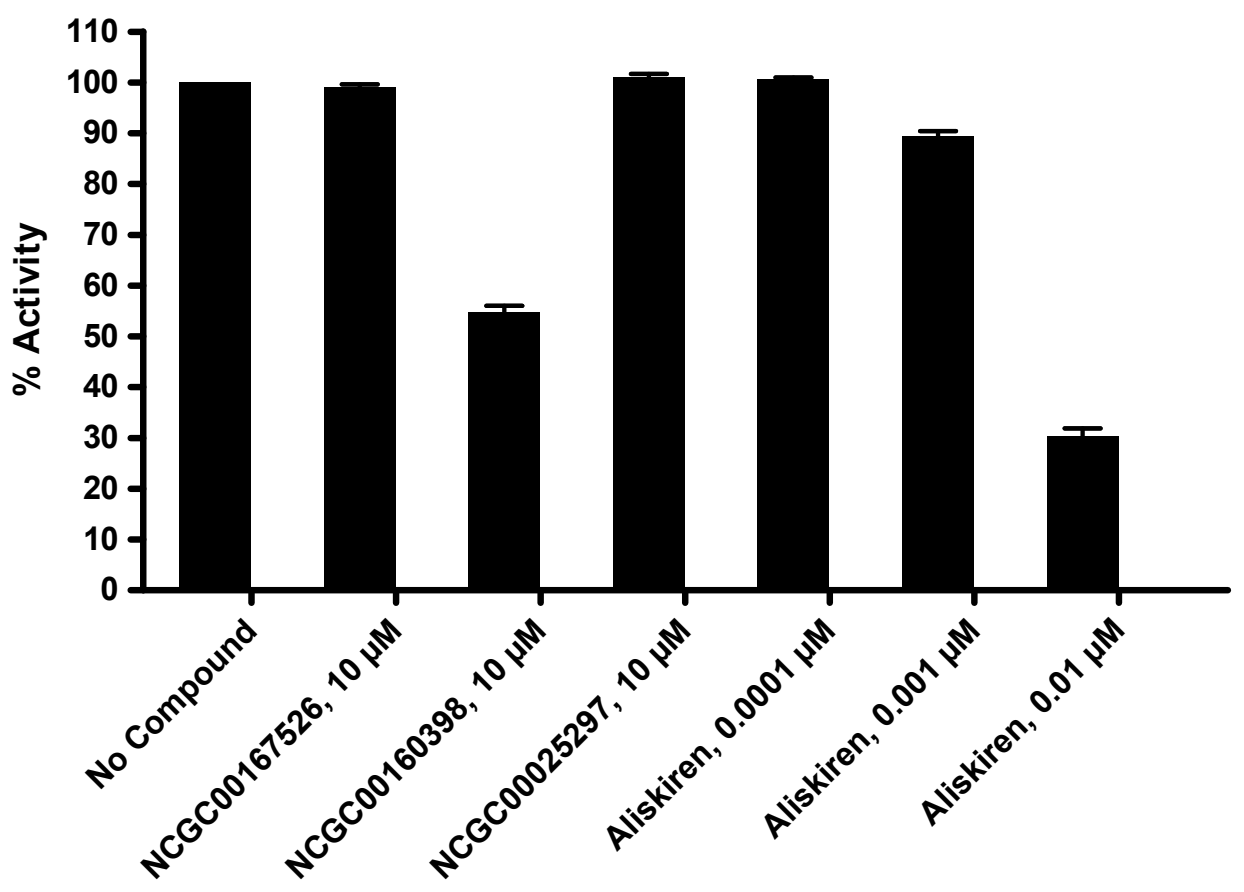




\section{Cornerstone Court West, Suite B}

San Diego, CA 92121

Tel: 1.858 .829 .3082

Fax: 1.858 .481 .8694

Email: info@bpsbioscience.com

\subsubsection{CL Protease}

Table 3.2.8. Data for the Effect of the Compounds on 3CL Protease Activity

\begin{tabular}{|c|c|c|c|c|c|c|c|}
\hline \multirow{2}{*}{ Compounds } & \multicolumn{2}{|c|}{$\begin{array}{l}\text { 3CL Proteae Activity } \\
\text { (Fluorescence count) }\end{array}$} & \multicolumn{2}{|c|}{$\begin{array}{l}\text { Background } \\
\text { (Fluorescence count) }\end{array}$} & \multicolumn{2}{|c|}{$\%$ Activity } & \multirow[t]{2}{*}{$\%$ Inhibition } \\
\hline & Repeat1 & Repeat2 & Repeat1 & Repeat2 & Repeat1 & Repeat2 & \\
\hline No Compound & 1171 & 1226 & 53 & 54 & 97 & 103 & 0 \\
\hline NCGC00167526, $10 \mu \mathrm{M}$ & 1123 & 1150 & 47 & 53 & 93 & 95 & 6 \\
\hline NCGC00160398, $10 \mu \mathrm{M}$ & 1198 & 1219 & 189 & 191 & 86 & 88 & 13 \\
\hline NCGC00025297, $10 \mu \mathrm{M}$ & 1171 & 1172 & 51 & 51 & 97 & 97 & 3 \\
\hline $\mathrm{GC} 376,0.05 \mu \mathrm{M}$ & 1125 & 1171 & 73 & 150 & 90 & 87 & 11 \\
\hline $\mathrm{GC} 376,0.5 \mu \mathrm{M}$ & 676 & 605 & 79 & 84 & 42 & 34 & 62 \\
\hline $\mathrm{GC} 376,5 \mu \mathrm{M}$ & 310 & 300 & 77 & 56 & 4 & 5 & 96 \\
\hline Background & 259 & 242 & 54 & 55 & & & \\
\hline
\end{tabular}

\section{CL Protease activity}

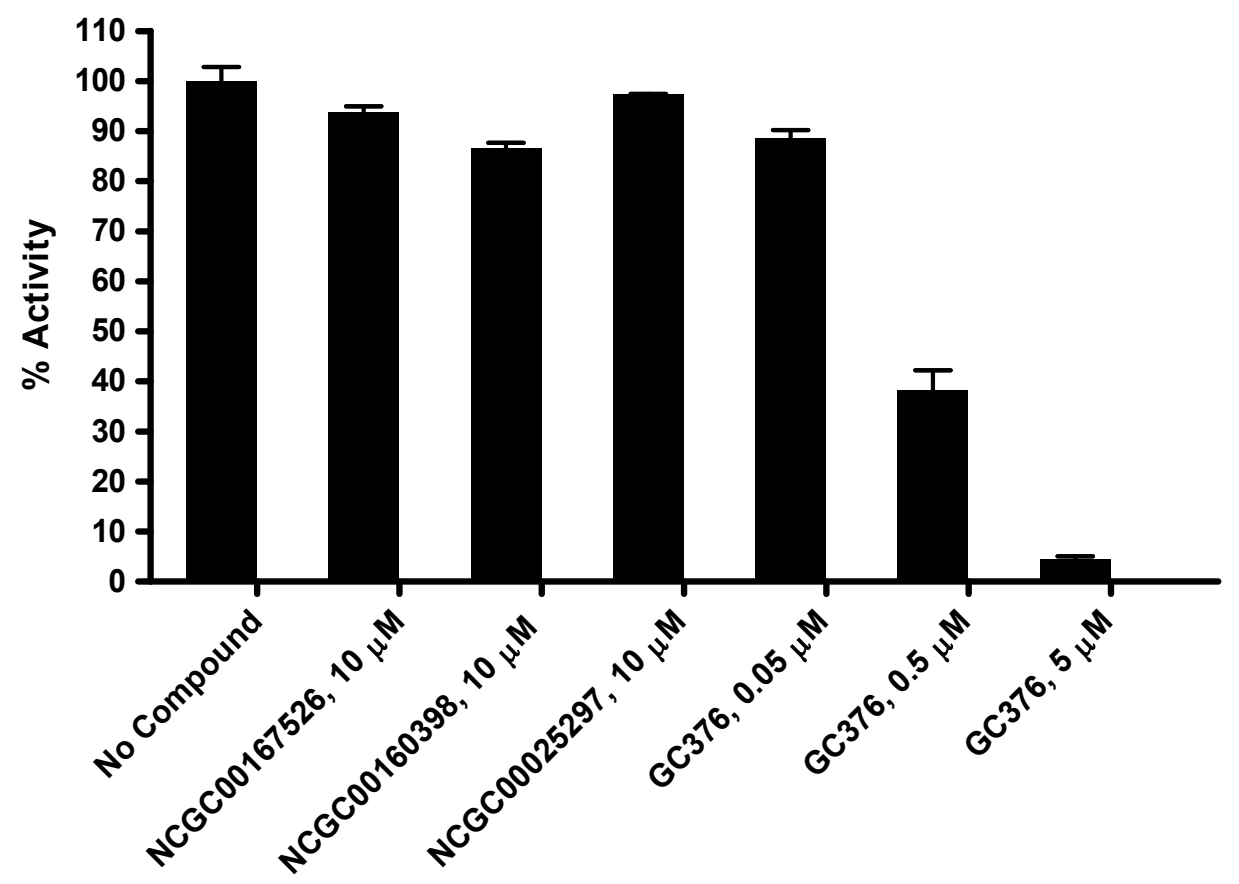


6042 Cornerstone Court West, Suite B

San Diego, CA 92121

Tel: 1.858 .829 .3082

Fax: 1.858 .481 .8694

Email: info@bpsbioscience.com

\subsection{9. $\underline{\mathrm{A} 20}$}

Table 3.2.9. Data for the Effect of the Compounds on A20 Activity

\begin{tabular}{|c|c|c|c|c|c|c|c|}
\hline \multirow{2}{*}{ Compounds } & \multicolumn{2}{|c|}{$\begin{array}{c}\text { A20 Activity } \\
\text { (Fluorescence count) }\end{array}$} & \multicolumn{2}{|c|}{$\begin{array}{c}\text { Background } \\
\text { (Fluorescence count) }\end{array}$} & \multicolumn{2}{|c|}{$\%$ Activity } & \multirow{2}{*}{$\%$ Inhibition } \\
\hline & Repeat1 & Repeat2 & Repeat1 & Repeat2 & Repeat1 & Repeat2 & \\
\hline No Compound & 192 & 189 & 22 & 22 & 101 & 99 & 0 \\
\hline NCGC00167526, $10 \mu \mathrm{M}$ & 183 & 183 & 22 & 21 & 95 & 96 & 4 \\
\hline NCGC00160398, $10 \mu \mathrm{M}$ & 221 & 221 & 75 & 74 & 86 & 87 & 13 \\
\hline NCGC00025297, $10 \mu \mathrm{M}$ & 183 & 183 & 21 & 20 & 96 & 97 & 4 \\
\hline Ub-Aldehyde, $0.001 \mu \mathrm{M}$ & 170 & 172 & 19 & 22 & 89 & 89 & 11 \\
\hline Ub-Aldehyde, $0.01 \mu \mathrm{M}$ & 101 & 99 & 21 & 21 & 47 & 45 & 54 \\
\hline Ub-Aldehyde, $0.1 \mu \mathrm{M}$ & 28 & 28 & 20 & 19 & 3 & 4 & 96 \\
\hline Background & 15 & 15 & 12 & 13 & & & \\
\hline
\end{tabular}

\section{A20 Activity}

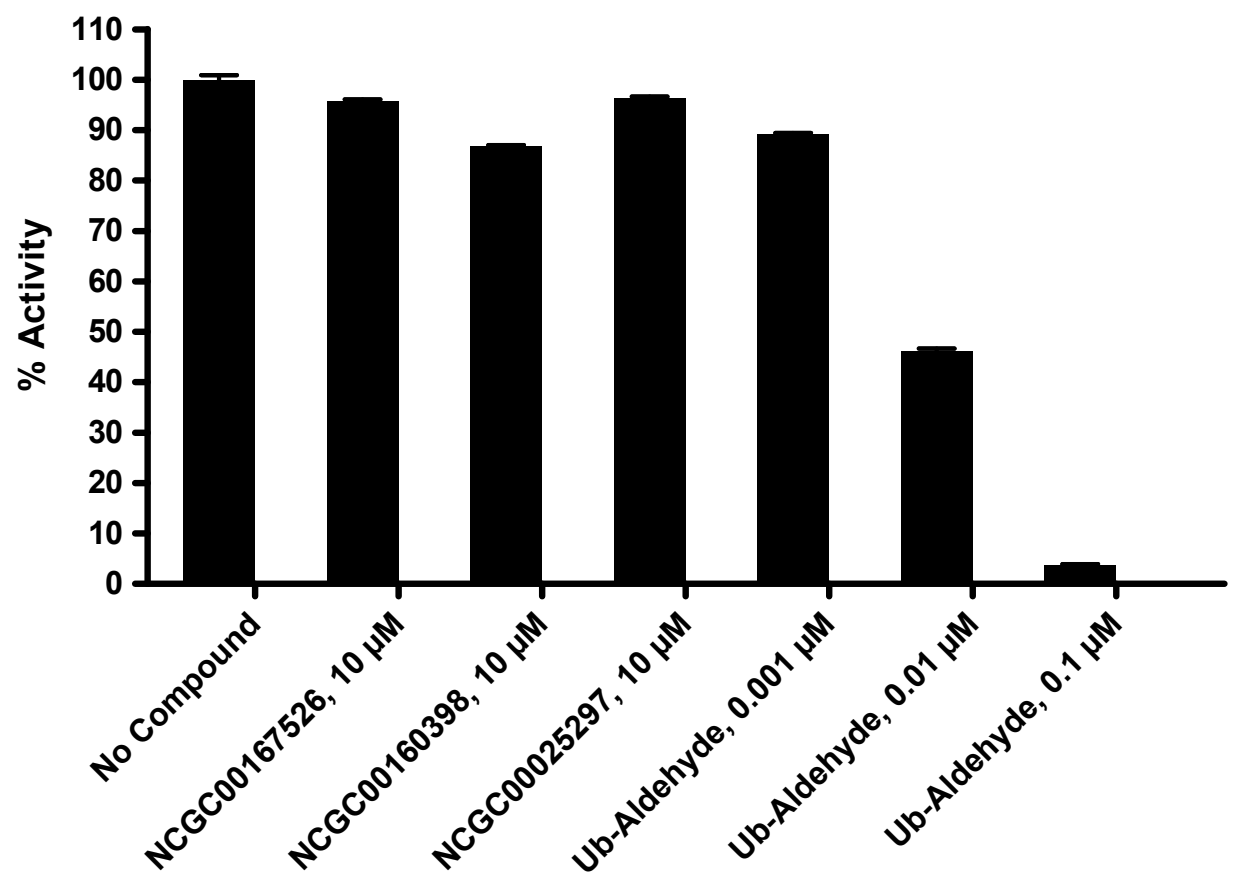




\section{Cornerstone Court West, Suite B \\ San Diego, CA 92121}

Tel: 1.858 .829 .3082

Fax: 1.858 .481 .8694

Email: info@bpsbioscience.com

\subsubsection{Ataxin-3}

Table 3.2.10. Data for the Effect of the Compounds on Ataxin-3 Activity

\begin{tabular}{|c|c|c|c|c|c|c|c|}
\hline \multirow[t]{2}{*}{ Compounds } & \multicolumn{2}{|c|}{$\begin{array}{l}\text { Ataxin-3 Activity } \\
\text { (Fluorescence count) }\end{array}$} & \multicolumn{2}{|c|}{$\begin{array}{c}\text { Background } \\
\text { (Fluorescence count) }\end{array}$} & \multicolumn{2}{|c|}{$\%$ Activity } & \multirow[t]{2}{*}{$\%$ Inhibition } \\
\hline & Repeat1 & Repeat2 & Repeat1 & Repeat2 & Repeat1 & Repeat2 & \\
\hline No Compound & 93 & 92 & 29 & 30 & 102 & 98 & 0 \\
\hline NCGC00167526, $10 \mu \mathrm{M}$ & 90 & 93 & 30 & 31 & 95 & 98 & 3 \\
\hline NCGC00160398, $10 \mu \mathrm{M}$ & 212 & 214 & 68 & 67 & 230 & 234 & 0 \\
\hline NCGC00025297, $10 \mu \mathrm{M}$ & 91 & 88 & 27 & 30 & 102 & 92 & 3 \\
\hline Ub-Aldehyde, $0.1 \mu \mathrm{M}$ & 73 & 73 & 28 & 30 & 71 & 68 & 30 \\
\hline Ub-Aldehyde, $1 \mu \mathrm{M}$ & 49 & 50 & 29 & 28 & 31 & 34 & 67 \\
\hline Ub-Aldehyde, $10 \mu \mathrm{M}$ & 26 & 26 & 29 & 29 & 4 & 4 & 96 \\
\hline Background & 15 & 16 & 16 & 16 & & & \\
\hline
\end{tabular}

\section{Ataxin-3 Activity}

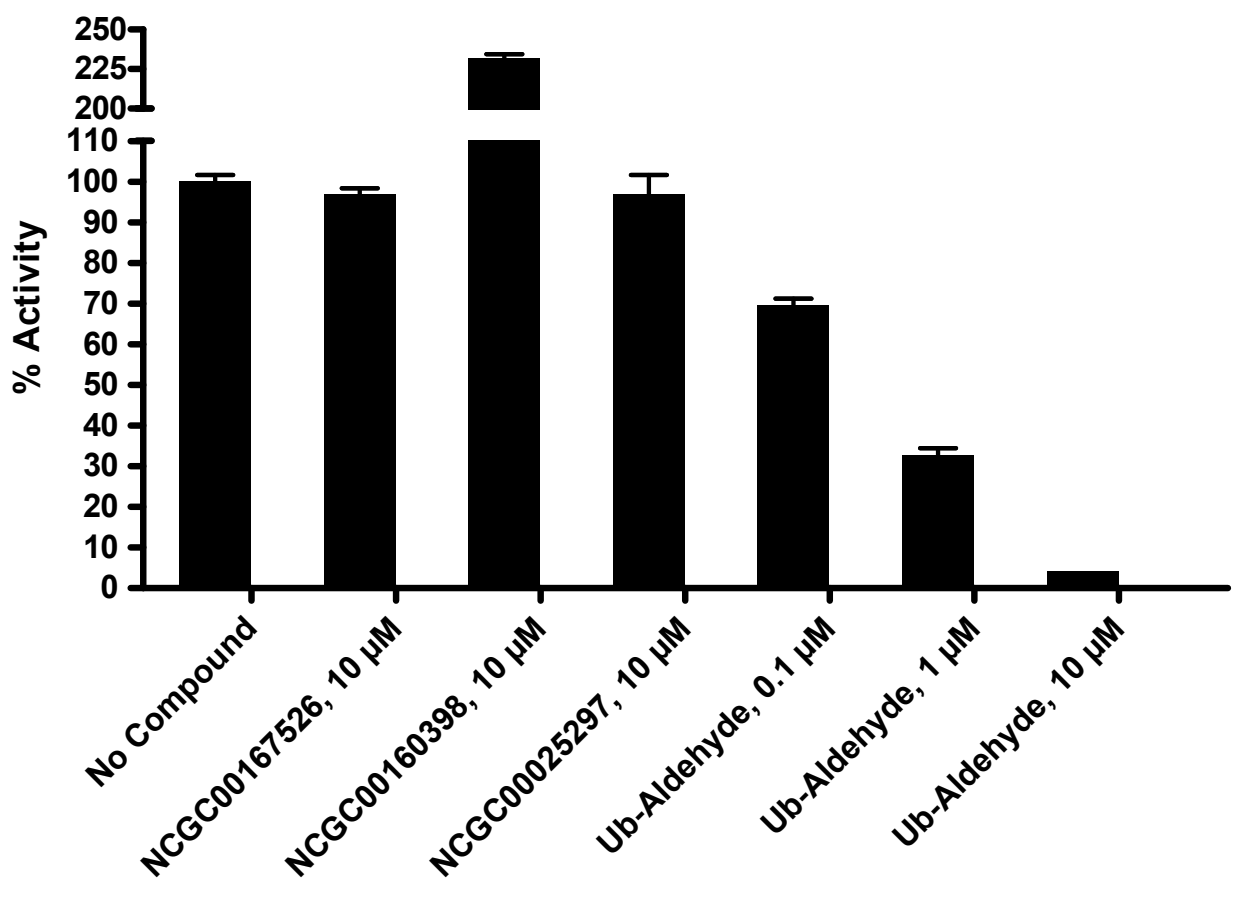


6042 Cornerstone Court West, Suite B

San Diego, CA 92121

Tel: 1.858 .829 .3082

Fax: 1.858 .481 .8694

Email: info@bpsbioscience.com

\subsubsection{Cathepsin B}

Table 3.2.11. Data for the Effect of the Compounds on Cathepsin B Activity

\begin{tabular}{|c|c|c|c|c|c|}
\hline \multirow[t]{2}{*}{ Compounds } & \multicolumn{2}{|c|}{$\begin{array}{l}\text { Cathepsin B Activity } \\
\text { (Fluorescence count) }\end{array}$} & \multicolumn{2}{|c|}{$\%$ Activity } & \multirow[t]{2}{*}{$\%$ Inhibition } \\
\hline & Repeat1 & Repeat2 & Repeat1 & Repeat2 & \\
\hline No Compound & 1818 & 2012 & 95 & 105 & 0 \\
\hline NCGC00167526, $10 \mu \mathrm{M}$ & 2032 & 2004 & 106 & 105 & 0 \\
\hline NCGC00160398, $10 \mu \mathrm{M}$ & 994 & 1056 & 50 & 53 & 48 \\
\hline NCGC00025297, $10 \mu \mathrm{M}$ & 1948 & 2033 & 102 & 106 & 0 \\
\hline E-64, $0.0005 \mu \mathrm{M}$ & 1274 & 1388 & 65 & 71 & 32 \\
\hline E-64, $0.005 \mu \mathrm{M}$ & 479 & 507 & 22 & 24 & 77 \\
\hline E-64, $0.05 \mu \mathrm{M}$ & 119 & 121 & 3 & 3 & 97 \\
\hline Background & 64 & 72 & & & \\
\hline
\end{tabular}

Cathepsin B Activity

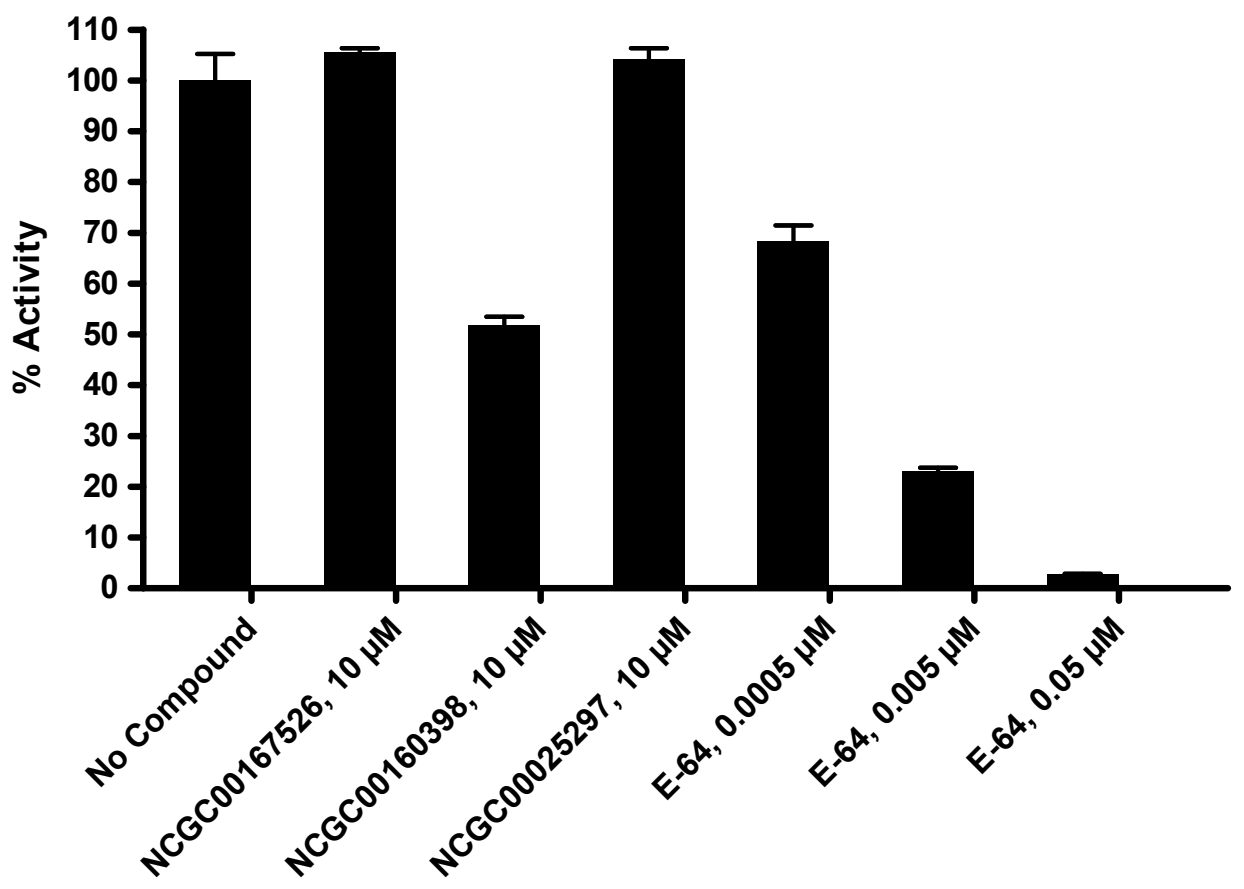


6042 Cornerstone Court West, Suite B

San Diego, CA 92121

Tel: 1.858 .829 .3082

Fax: 1.858.481.8694

Email: info@bpsbioscience.com

3.2.12. Cathepsin F

Table 3.2.12. Data for the Effect of the Compounds on Cathepsin F Activity

\begin{tabular}{|c|c|c|c|c|c|}
\hline \multirow[t]{2}{*}{ Compounds } & \multicolumn{2}{|c|}{$\begin{array}{l}\text { Cathepsin F Activity } \\
\text { (Fluorescence count) }\end{array}$} & \multicolumn{2}{|c|}{$\%$ Activity } & \multirow[t]{2}{*}{$\%$ Inhibition } \\
\hline & Repeat1 & Repeat2 & Repeat1 & Repeat2 & \\
\hline No Compound & 857 & 852 & 100 & 100 & 0 \\
\hline NCGC00167526, $10 \mu \mathrm{M}$ & 870 & 858 & 102 & 101 & 0 \\
\hline NCGC00160398, $10 \mu \mathrm{M}$ & 855 & 868 & 100 & 102 & 0 \\
\hline NCGC00025297, $10 \mu \mathrm{M}$ & 843 & 839 & 98 & 98 & 2 \\
\hline Cystatin $\mathrm{C}, 0.1 \mu \mathrm{M}$ & 724 & 705 & 81 & 78 & 20 \\
\hline Cystatin $\mathrm{C}, 1 \mu \mathrm{M}$ & 370 & 320 & 30 & 23 & 73 \\
\hline Cystatin C, $10 \mu \mathrm{M}$ & 232 & 247 & 10 & 12 & 89 \\
\hline Background & 164 & 158 & & & \\
\hline
\end{tabular}

Cathepsin F Activity

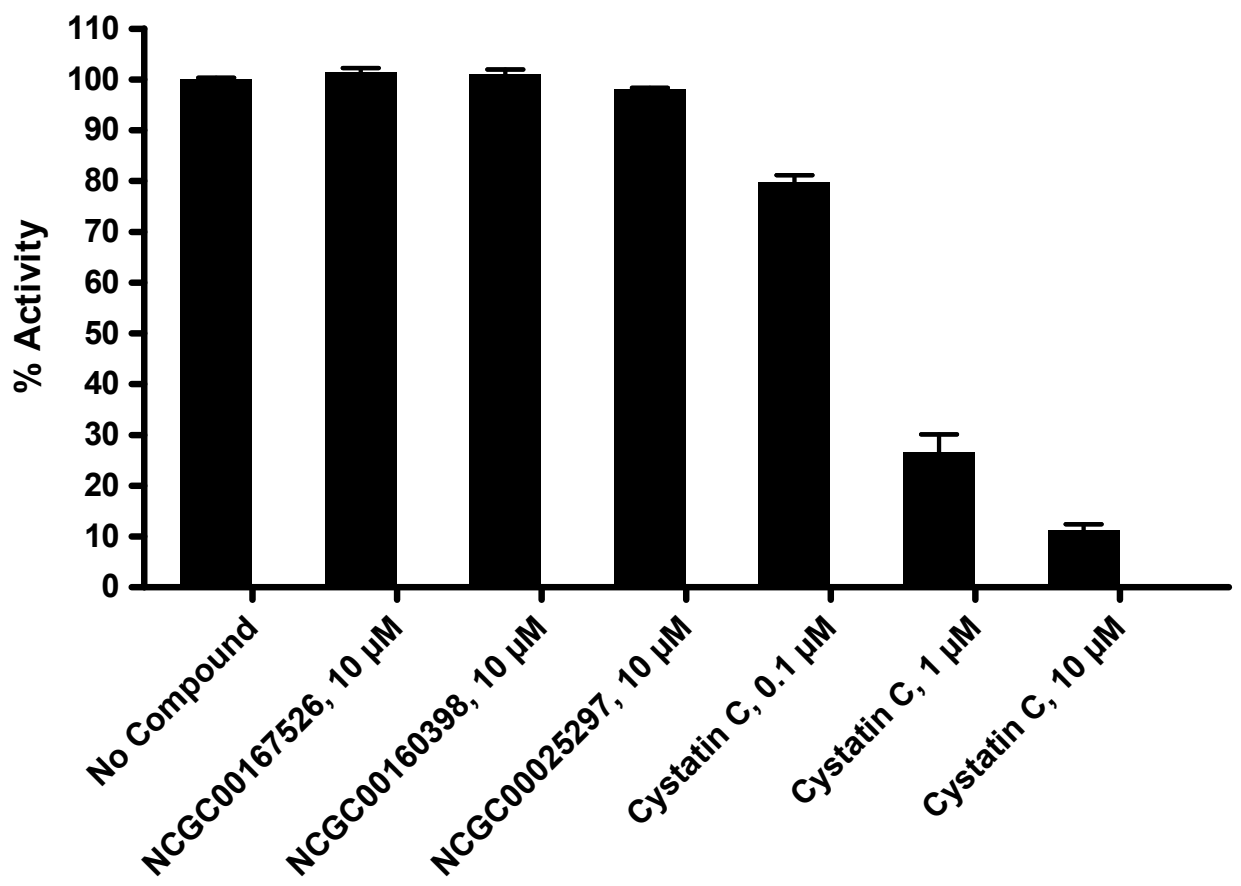


6042 Cornerstone Court West, Suite B

San Diego, CA 92121

Tel: 1.858 .829 .3082

Fax: 1.858 .481 .8694

Email: info@bpsbioscience.com

\subsubsection{Cathepsin L}

Table 3.2.13. Data for the Effect of the Compounds on Cathepsin L Activity

\begin{tabular}{|c|c|c|c|c|c|}
\hline \multirow[t]{2}{*}{ Compounds } & \multicolumn{2}{|c|}{$\begin{array}{l}\text { Cathepsin L Activity } \\
\text { (Fluorescence count) }\end{array}$} & \multicolumn{2}{|c|}{$\%$ Activity } & \multirow[t]{2}{*}{$\%$ Inhibition } \\
\hline & Repeat1 & Repeat2 & Repeat1 & Repeat2 & \\
\hline No Compound & 3581 & 3625 & 99 & 101 & 0 \\
\hline NCGC00167526, $10 \mu \mathrm{M}$ & 3571 & 3486 & 99 & 97 & 2 \\
\hline NCGC00160398, $10 \mu \mathrm{M}$ & 1563 & 1606 & 42 & 44 & 57 \\
\hline NCGC00025297, $10 \mu \mathrm{M}$ & 3693 & 3583 & 103 & 99 & 0 \\
\hline E-64, $0.0005 \mu \mathrm{M}$ & 3128 & 3141 & 87 & 87 & 13 \\
\hline E-64, $0.005 \mu \mathrm{M}$ & 2209 & 2271 & 61 & 62 & 39 \\
\hline E-64, $0.05 \mu \mathrm{M}$ & 629 & 663 & 16 & 17 & 84 \\
\hline Background & 64 & 69 & & & \\
\hline
\end{tabular}

Cathepsin L Activity

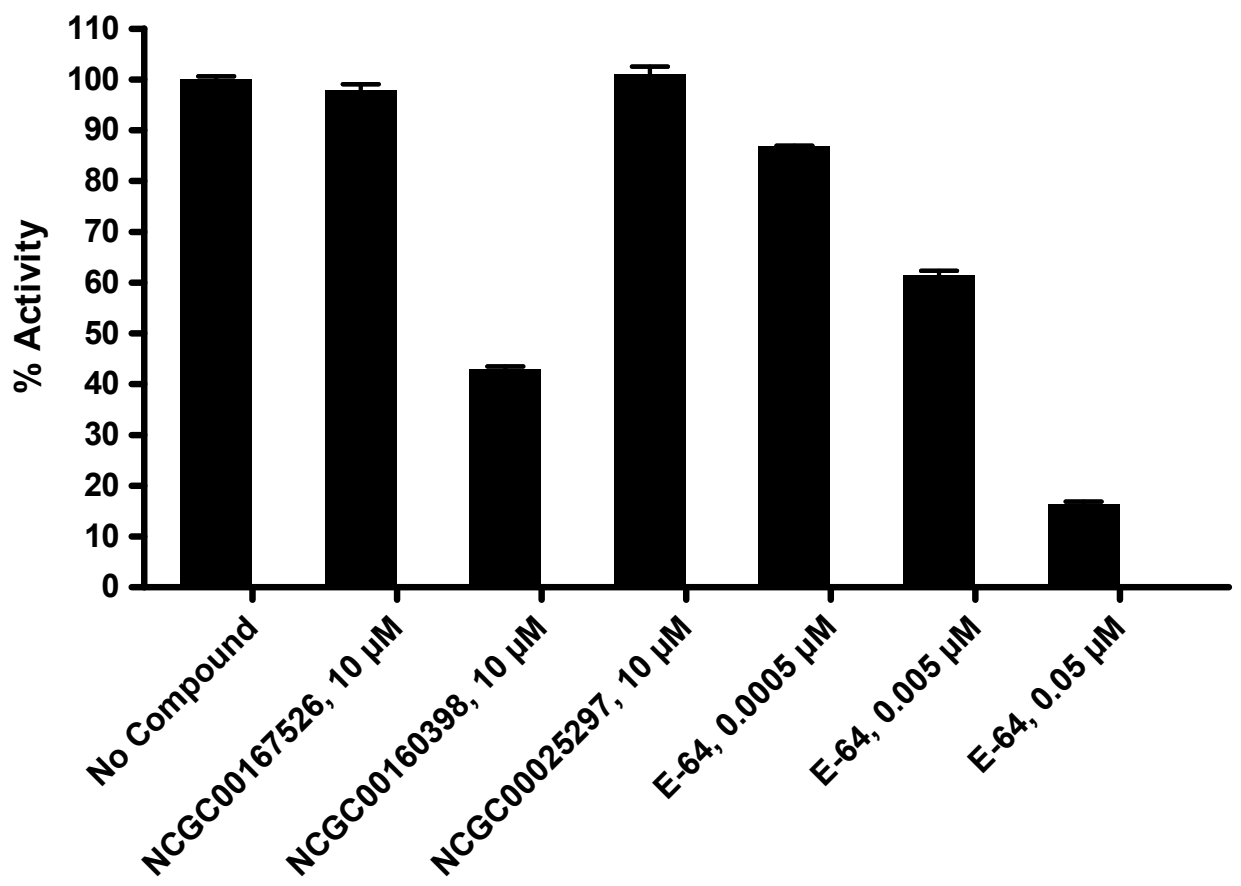




\section{Cornerstone Court West, Suite B}

San Diego, CA 92121

Tel: 1.858 .829 .3082

Fax: 1.858 .481 .8694

Email: info@bpsbioscience.com

\subsubsection{Cathepsin $\mathrm{S}$}

Table 3.2.14. Data for the Effect of the Compounds on Cathepsin S Activity

\begin{tabular}{|c|c|c|c|c|c|}
\hline \multirow[t]{2}{*}{ Compounds } & \multicolumn{2}{|c|}{$\begin{array}{l}\text { Cathepsin S Activity } \\
\text { (Fluorescence count) }\end{array}$} & \multicolumn{2}{|c|}{$\%$ Activity } & \multirow[t]{2}{*}{$\%$ Inhibition } \\
\hline & Repeat1 & Repeat2 & Repeat1 & Repeat2 & \\
\hline No Compound & 15416 & 15602 & 99 & 101 & 0 \\
\hline NCGC00167526, $10 \mu \mathrm{M}$ & 15033 & 15041 & 97 & 97 & 3 \\
\hline NCGC00160398, $10 \mu \mathrm{M}$ & 14129 & 14453 & 91 & 93 & 8 \\
\hline $\mathrm{NCGC} 00025297,10 \mu \mathrm{M}$ & 14932 & 14929 & 96 & 96 & 4 \\
\hline E-64, $0.005 \mu \mathrm{M}$ & 12846 & 12674 & 83 & 82 & 18 \\
\hline E-64, $0.05 \mu \mathrm{M}$ & 3066 & 3005 & 19 & 19 & 81 \\
\hline E-64, $0.5 \mu \mathrm{M}$ & 515 & 575 & 3 & 3 & 97 \\
\hline Background & 64 & 70 & & & \\
\hline
\end{tabular}

Cathepsin S Activity

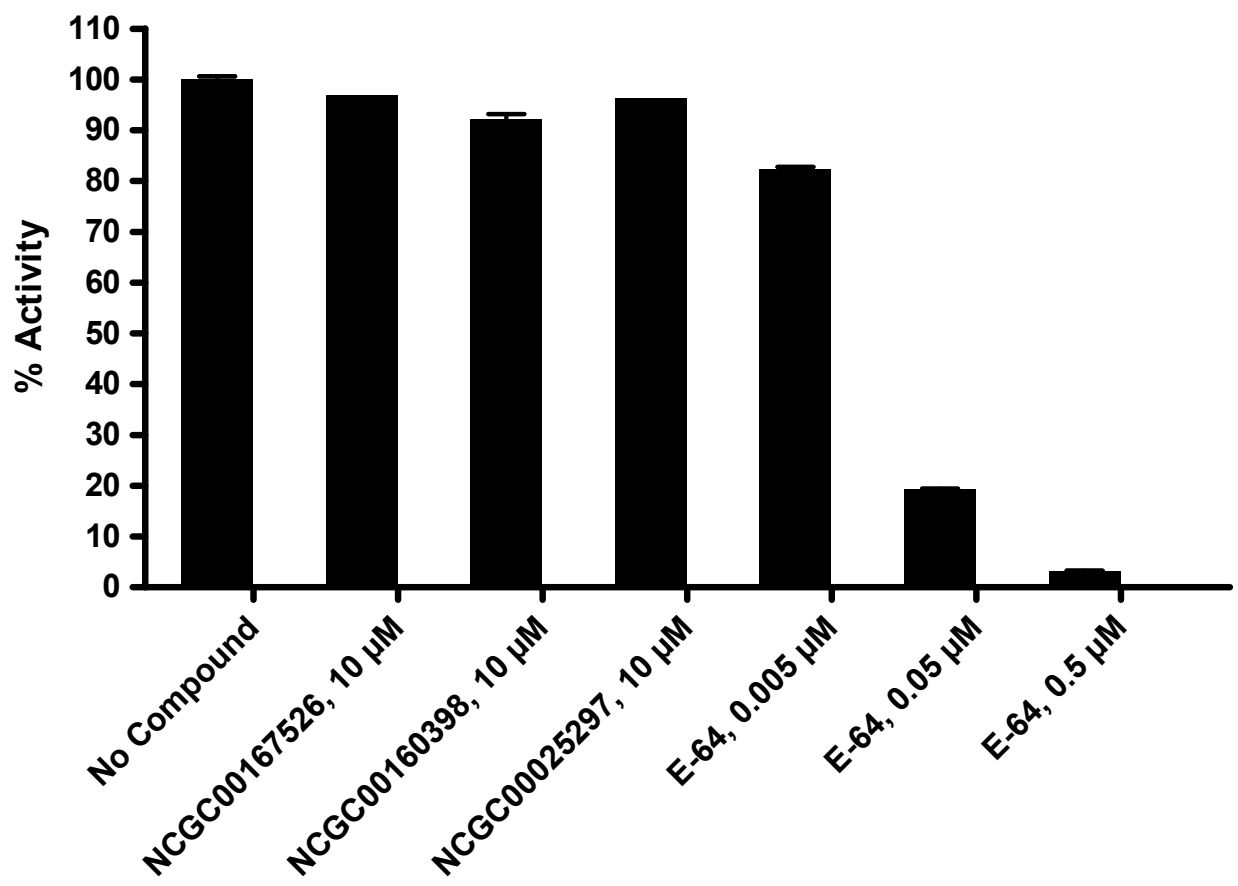


6042 Cornerstone Court West, Suite B

San Diego, CA 92121

Tel: 1.858 .829 .3082

Fax: 1.858.481.8694

Email: info@bpsbioscience.com

\subsubsection{Cathepsin V}

Table 3.2.15. Data for the Effect of the Compounds on Cathepsin V Activity

\begin{tabular}{|c|c|c|c|c|c|}
\hline \multirow[t]{2}{*}{ Compounds } & \multicolumn{2}{|c|}{$\begin{array}{l}\text { Cathepsin V Activity } \\
\text { (Fluorescence count) }\end{array}$} & \multicolumn{2}{|c|}{$\%$ Activity } & \multirow[t]{2}{*}{$\%$ Inhibition } \\
\hline & Repeat1 & Repeat2 & Repeat1 & Repeat2 & \\
\hline No Compound & 6640 & 6458 & 101 & 99 & 0 \\
\hline NCGC00167526, $10 \mu \mathrm{M}$ & 6463 & 6533 & 99 & 100 & 1 \\
\hline NCGC00160398, $10 \mu \mathrm{M}$ & 4748 & 4883 & 72 & 74 & 27 \\
\hline NCGC00025297, $10 \mu \mathrm{M}$ & 6423 & 6337 & 98 & 97 & 3 \\
\hline E-64, $0.005 \mu \mathrm{M}$ & 5488 & 5430 & 84 & 83 & 17 \\
\hline E-64, $0.05 \mu \mathrm{M}$ & 2947 & 2798 & 45 & 42 & 57 \\
\hline E-64, $0.5 \mu \mathrm{M}$ & 518 & 407 & 7 & 6 & 94 \\
\hline Background & 45 & 43 & & & \\
\hline
\end{tabular}

Cathepsin V Activity

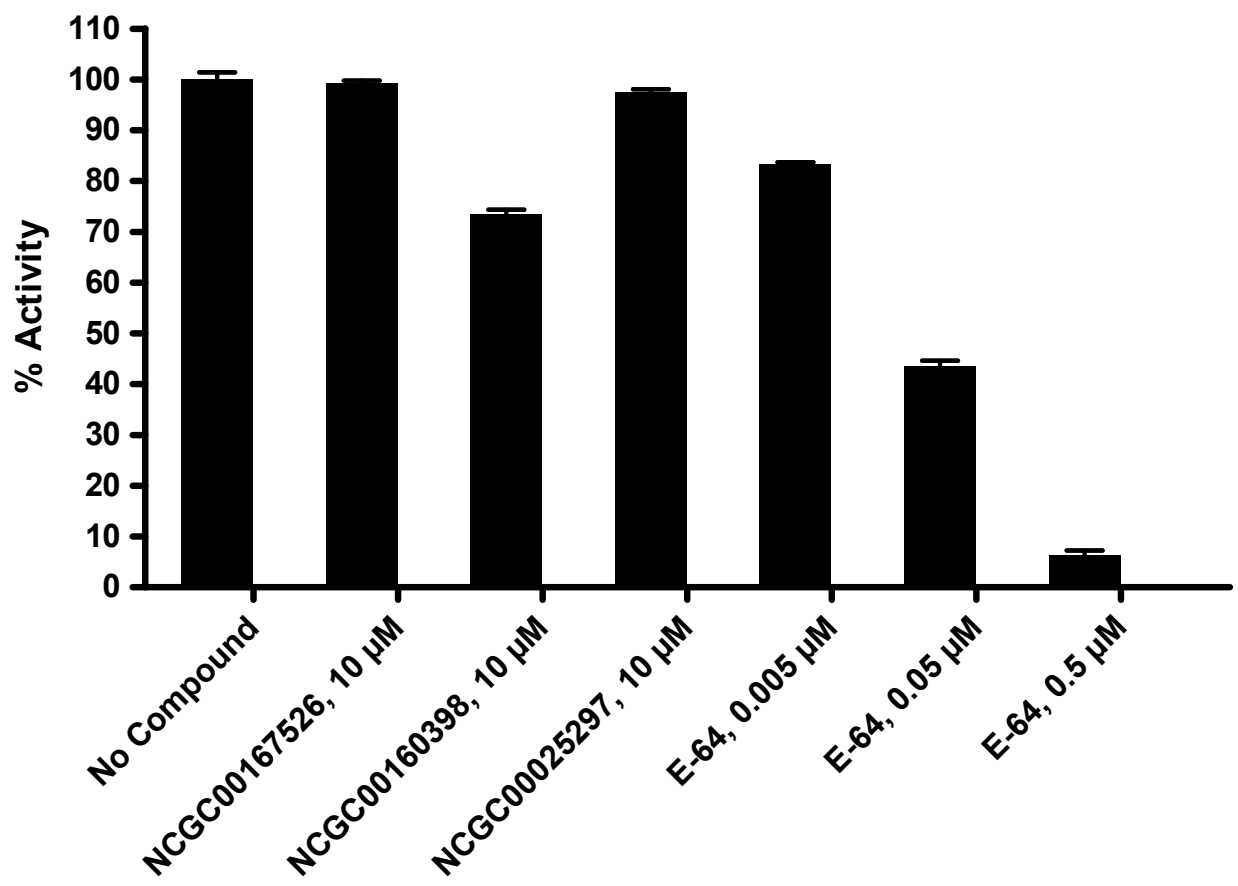


6042 Cornerstone Court West, Suite B

San Diego, CA 92121

Tel: 1.858 .829 .3082

Fax: 1.858 .481 .8694

Email: info@bpsbioscience.com

\subsubsection{MALT1}

Table 3.2.16. Data for the Effect of the Compounds on MALT1 Activity

\begin{tabular}{|c|c|c|c|c|c|c|c|}
\hline \multirow{2}{*}{ Compounds } & \multicolumn{2}{|c|}{$\begin{array}{l}\text { MALT1 Activity } \\
\text { (Fluorescence count) }\end{array}$} & \multicolumn{2}{|c|}{$\begin{array}{c}\text { Background } \\
\text { (Fluorescence count) }\end{array}$} & \multicolumn{2}{|c|}{$\%$ Activity } & \multirow[t]{2}{*}{$\%$ Inhibition } \\
\hline & Repeat1 & Repeat2 & Repeat1 & Repeat2 & Repeat1 & Repeat2 & \\
\hline No Compound & 7256 & 7345 & 450 & 544 & 100 & 100 & 0 \\
\hline NCGC00167526, $10 \mu \mathrm{M}$ & 3957 & 3934 & 361 & 325 & 52 & 52 & 48 \\
\hline NCGC00160398, $10 \mu \mathrm{M}$ & 4041 & 4049 & 500 & 546 & 51 & 50 & 49 \\
\hline NCGC00025297, $10 \mu \mathrm{M}$ & 4120 & 4121 & 382 & 348 & 54 & 55 & 46 \\
\hline Z-VRPR-FMK, $0.001 \mu \mathrm{M}$ & 6564 & 6756 & 400 & 514 & 90 & 92 & 9 \\
\hline Z-VRPR-FMK, $0.01 \mu \mathrm{M}$ & 3396 & 3379 & 295 & 344 & 44 & 43 & 56 \\
\hline Z-VRPR-FMK, $0.1 \mu \mathrm{M}$ & 668 & 669 & 278 & 369 & 4 & 2 & 97 \\
\hline Background & 404 & 405 & 264 & 272 & & & \\
\hline
\end{tabular}

MALT1 Activity

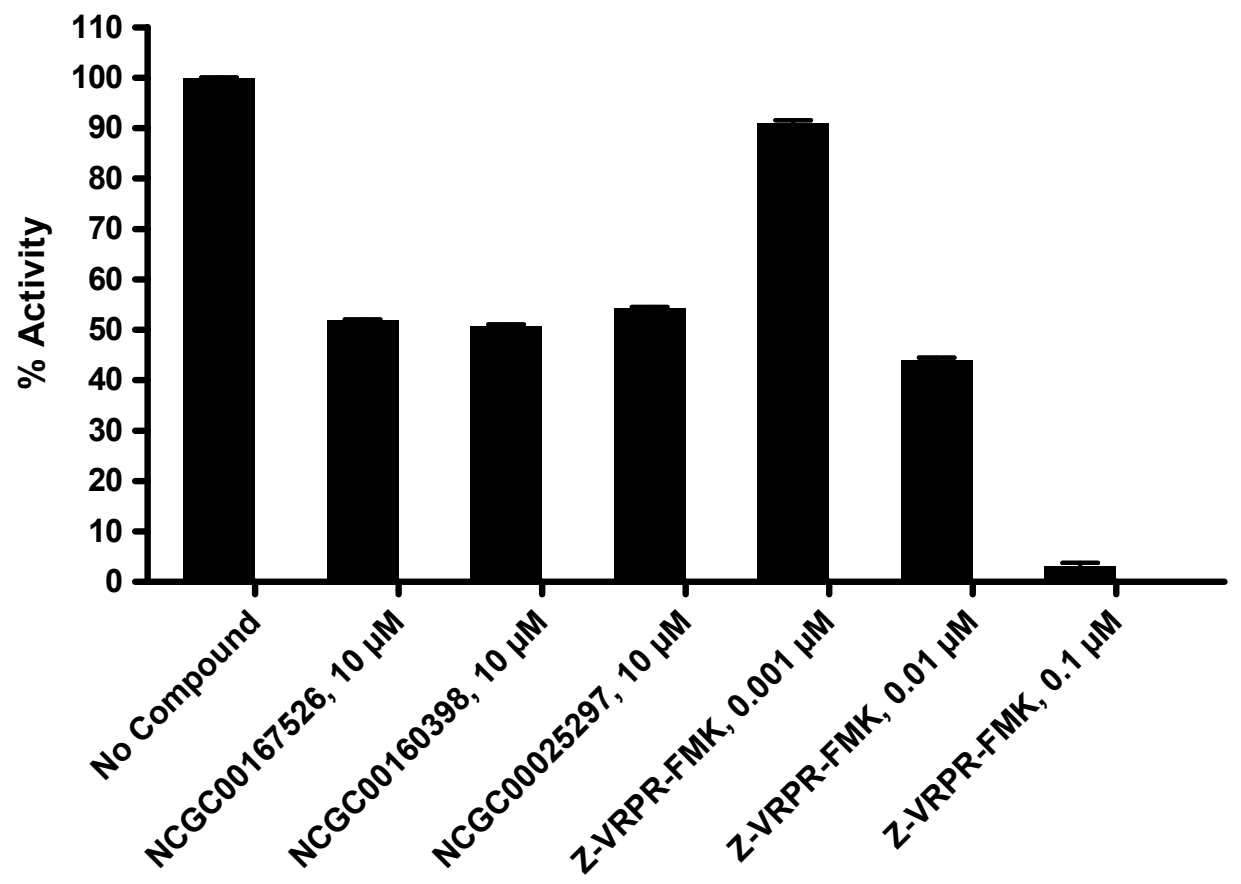


6042 Cornerstone Court West, Suite B

San Diego, CA 92121

Tel: 1.858 .829 .3082

Fax: 1.858 .481 .8694

Email: info@bpsbioscience.com

\subsubsection{OTUD6Bc}

Table 3.2.17. Data for the Effect of the Compounds on OTUD6Bc Activity

\begin{tabular}{|c|c|c|c|c|c|c|c|}
\hline \multirow{2}{*}{ Compounds } & \multicolumn{2}{|c|}{$\begin{array}{l}\text { OTUD6Bc Activity } \\
\text { (Fluorescence count) }\end{array}$} & \multicolumn{2}{|c|}{$\begin{array}{c}\text { Background } \\
\text { (Fluorescence count) }\end{array}$} & \multicolumn{2}{|c|}{$\%$ Activity } & \multirow[t]{2}{*}{$\%$ Inhibition } \\
\hline & Repeat1 & Repeat2 & Repeat1 & Repeat2 & Repeat1 & Repeat2 & \\
\hline No Compound & 224 & 225 & 22 & 22 & 100 & 100 & 0 \\
\hline NCGC00167526, $10 \mu \mathrm{M}$ & 222 & 220 & 21 & 20 & 99 & 99 & 1 \\
\hline NCGC00160398, $10 \mu \mathrm{M}$ & 265 & 260 & 80 & 77 & 91 & 90 & 9 \\
\hline NCGC00025297, $10 \mu \mathrm{M}$ & 218 & 217 & 20 & 21 & 98 & 97 & 3 \\
\hline Ub-Aldehyde, $0.01 \mu \mathrm{M}$ & 218 & 205 & 22 & 21 & 97 & 91 & 6 \\
\hline Ub-Aldehyde, $0.1 \mu \mathrm{M}$ & 176 & 176 & 20 & 21 & 77 & 76 & 24 \\
\hline Ub-Aldehyde, $1 \mu \mathrm{M}$ & 17 & 18 & 16 & 18 & 0 & 0 & 100 \\
\hline Background & 16 & 16 & 14 & 13 & & & \\
\hline
\end{tabular}

OTUD6Bc Activity

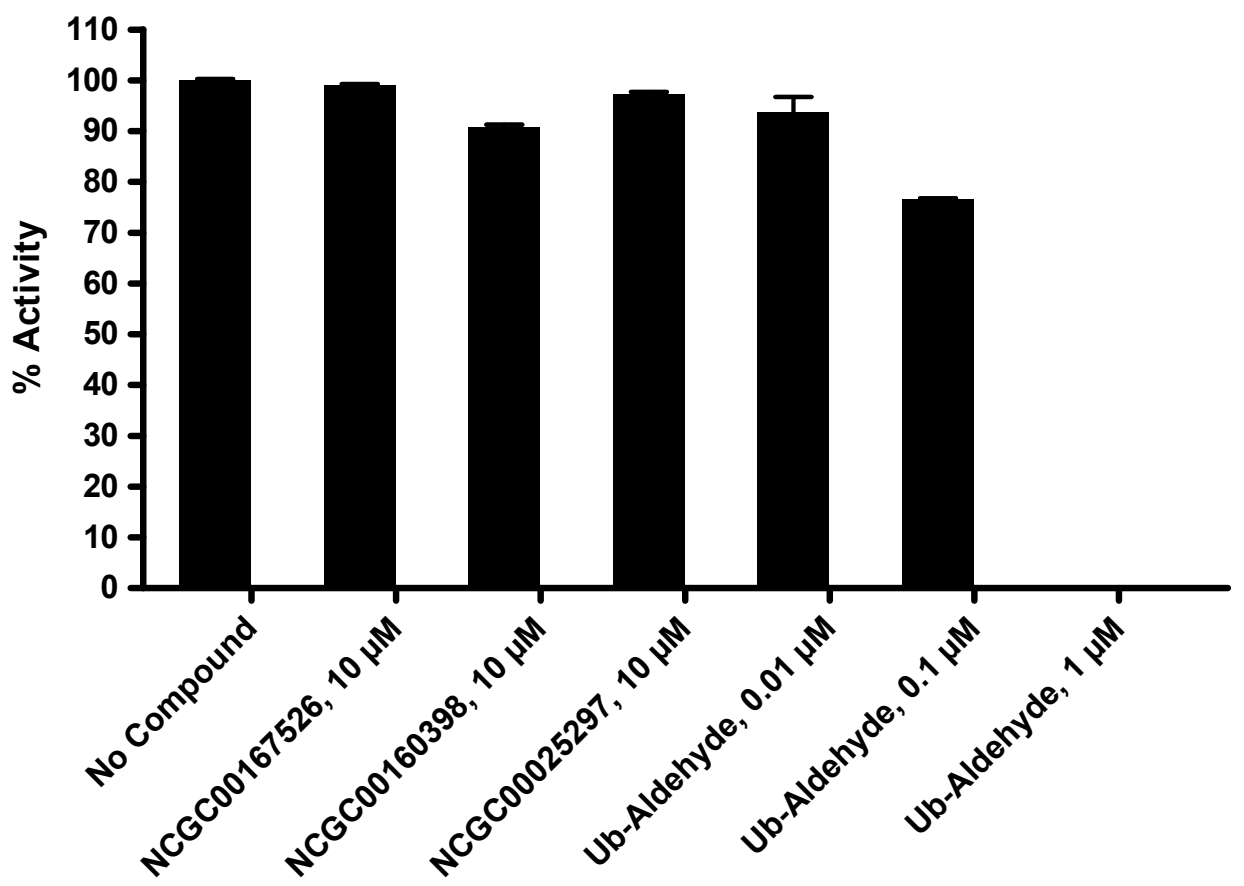


6042 Cornerstone Court West, Suite B

San Diego, CA 92121

Tel: 1.858 .829 .3082

Fax: 1.858 .481 .8694

Email: info@bpsbioscience.com

3.2.18. UCHL1

Table 3.2.18. Data for the Effect of the Compounds on UCHL1 Activity

\begin{tabular}{|c|c|c|c|c|c|c|c|}
\hline \multirow{2}{*}{$\begin{array}{c}\text { Compounds } \\
\text { No Compound }\end{array}$} & $\begin{array}{c}\text { OCHL1 Activity } \\
\text { (Fluorescence count) }\end{array}$ & $\begin{array}{c}\text { Background } \\
\text { (Fluorescence count) }\end{array}$ & \multicolumn{2}{|c|}{ \% Activity } & \% Inhibition \\
\cline { 2 - 6 } Repeat1 & Repeat2 & Repeat1 & Repeat2 & Repeat1 & Repeat2 & \\
\hline NCGC00167526, $10 \mu \mathrm{M}$ & 230 & 230 & 23 & 23 & 100 & 100 & 0 \\
\hline NCGC00160398, $10 \mu \mathrm{M}$ & 296 & 295 & 64 & 62 & 112 & 113 & 0 \\
\hline NCGC00025297, $10 \mu \mathrm{M}$ & 218 & 219 & 23 & 22 & 94 & 95 & 5 \\
\hline Ub-Aldehyde, $0.001 \mu \mathrm{M}$ & 213 & 217 & 21 & 22 & 93 & 94 & 7 \\
\hline Ub-Aldehyde, $0.01 \mu \mathrm{M}$ & 187 & 185 & 21 & 20 & 80 & 80 & 20 \\
\hline Ub-Aldehyde, $0.1 \mu \mathrm{M}$ & 80 & 81 & 21 & 21 & 29 & 29 & 71 \\
\hline Background & 14 & 14 & 14 & 14 & & & 93 \\
\hline
\end{tabular}

UCHL1 Activity

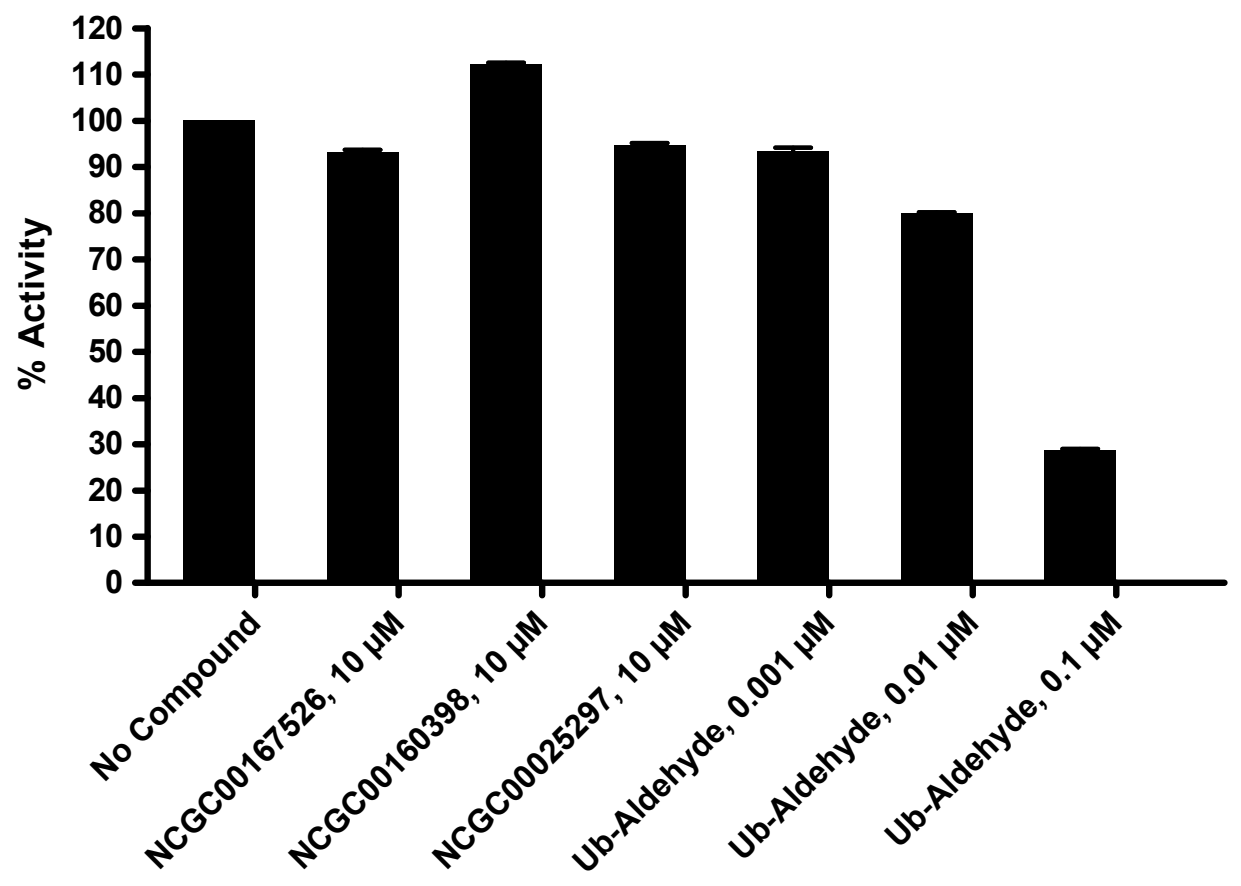


6042 Cornerstone Court West, Suite B

San Diego, CA 92121

Tel: 1.858 .829 .3082

Fax: 1.858 .481 .8694

Email: info@bpsbioscience.com

3.2.19. UCHL3

Table 3.2.19. Data for the Effect of the Compounds on UCHL3 Activity

\begin{tabular}{|c|c|c|c|c|c|c|c|}
\hline \multirow[t]{2}{*}{ Compounds } & \multicolumn{2}{|c|}{$\begin{array}{l}\text { OCHL3 Activity } \\
\text { (Fluorescence count) }\end{array}$} & \multicolumn{2}{|c|}{$\begin{array}{l}\text { Background } \\
\text { (Fluorescence count) }\end{array}$} & \multicolumn{2}{|c|}{$\%$ Activity } & \multirow[t]{2}{*}{$\%$ Inhibition } \\
\hline & Repeat1 & Repeat2 & Repeat1 & Repeat2 & Repeat1 & Repeat2 & \\
\hline No Compound & 634 & 626 & 49 & 48 & 101 & 99 & 0 \\
\hline NCGC00167526, $10 \mu \mathrm{M}$ & 615 & 611 & 48 & 46 & 97 & 97 & 3 \\
\hline NCGC00160398, $10 \mu \mathrm{M}$ & 647 & 647 & 98 & 97 & 94 & 95 & 6 \\
\hline NCGC00025297, $10 \mu \mathrm{M}$ & 607 & 610 & 48 & 49 & 96 & 96 & 4 \\
\hline Ub-Aldehyde, $0.0001 \mu \mathrm{M}$ & 600 & 608 & 44 & 46 & 96 & 97 & 4 \\
\hline Ub-Aldehyde, $0.001 \mu \mathrm{M}$ & 440 & 440 & 46 & 49 & 68 & 67 & 33 \\
\hline Ub-Aldehyde, $0.01 \mu \mathrm{M}$ & 111 & 112 & 40 & 42 & 12 & 11 & 89 \\
\hline Background & 19 & 18 & 14 & 14 & & & \\
\hline
\end{tabular}

UCHL3 Activity

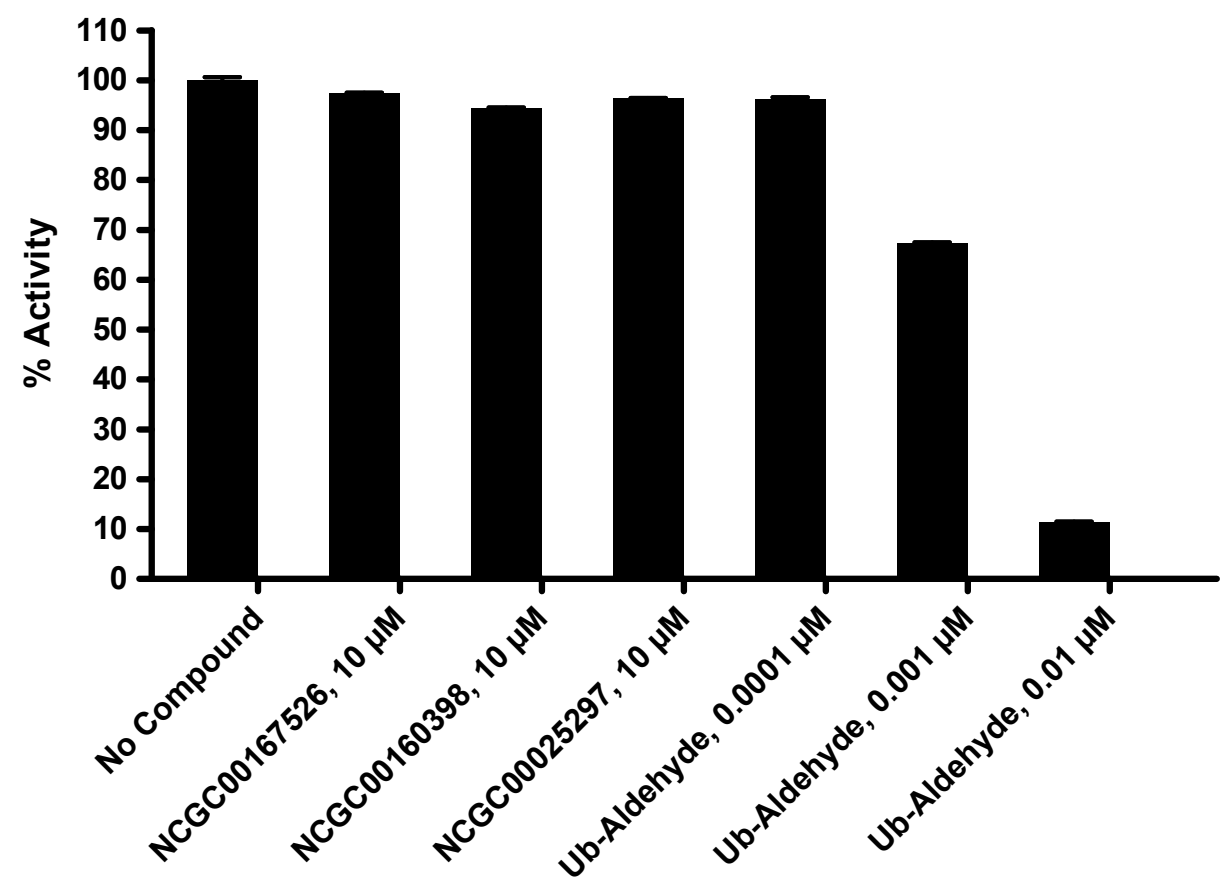


6042 Cornerstone Court West, Suite B

San Diego, CA 92121

Tel: 1.858 .829 .3082

Fax: 1.858 .481 .8694

Email: info@bpsbioscience.com

\subsubsection{USP2}

Table 3.2.20. Data for the Effect of the Compounds on USP2 Activity

\begin{tabular}{|c|c|c|c|c|c|c|c|}
\hline \multirow[t]{2}{*}{ Compounds } & \multicolumn{2}{|c|}{$\begin{array}{c}\text { USP2 Activity } \\
\text { (Fluorescence count) }\end{array}$} & \multicolumn{2}{|c|}{$\begin{array}{c}\text { Background } \\
\text { (Fluorescence count) }\end{array}$} & \multicolumn{2}{|c|}{$\%$ Activity } & \multirow[t]{2}{*}{$\%$ Inhibitior } \\
\hline & Repeat1 & Repeat2 & Repeat1 & Repeat2 & Repeat1 & Repeat2 & \\
\hline No Compound & 343 & 330 & 23 & 21 & 102 & 98 & 0 \\
\hline NCGC00167526, $10 \mu \mathrm{M}$ & 332 & 337 & 22 & 23 & 99 & 100 & 1 \\
\hline NCGC00160398, $10 \mu \mathrm{M}$ & 404 & 400 & 69 & 67 & 107 & 106 & 0 \\
\hline NCGC00025297, $10 \mu \mathrm{M}$ & 331 & 333 & 23 & 22 & 98 & 99 & 2 \\
\hline Ub-Aldehyde, $0.01 \mu \mathrm{M}$ & 324 & 325 & 24 & 27 & 95 & 95 & 5 \\
\hline Ub-Aldehyde, $0.1 \mu \mathrm{M}$ & 163 & 166 & 23 & 23 & 44 & 45 & 55 \\
\hline Ub-Aldehyde, $1 \mu \mathrm{M}$ & 32 & 33 & 20 & 21 & 4 & 4 & 96 \\
\hline Background & 15 & 14 & 14 & 13 & & & \\
\hline
\end{tabular}

USP2 Activity

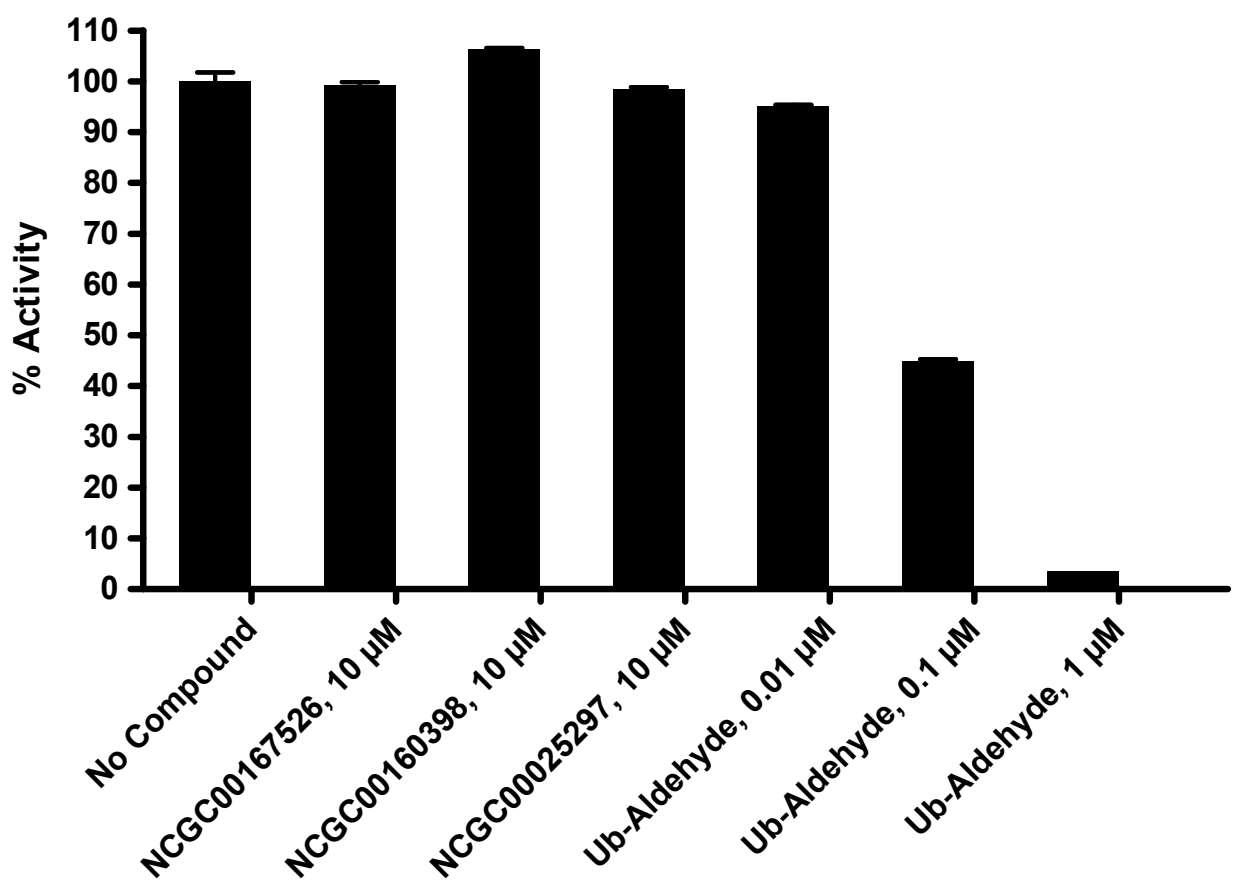


6042 Cornerstone Court West, Suite B

San Diego, CA 92121

Tel: 1.858 .829 .3082

Fax: 1.858 .481 .8694

Email: info@bpsbioscience.com

\subsubsection{USP5}

Table 3.2.21. Data for the Effect of the Compounds on USP5 Activity

\begin{tabular}{|c|c|c|c|c|c|c|c|}
\hline \multirow[t]{2}{*}{ Compounds } & \multicolumn{2}{|c|}{$\begin{array}{c}\text { USP5 Activity } \\
\text { (Fluorescence count) }\end{array}$} & \multicolumn{2}{|c|}{$\begin{array}{c}\text { Background } \\
\text { (Fluorescence count) }\end{array}$} & \multicolumn{2}{|c|}{$\%$ Activity } & \multirow[t]{2}{*}{$\%$ Inhibitior } \\
\hline & Repeat1 & Repeat2 & Repeat1 & Repeat2 & Repeat1 & Repeat2 & \\
\hline No Compound & 854 & 867 & 21 & 22 & 99 & 101 & 0 \\
\hline NCGC00167526, $10 \mu \mathrm{M}$ & 835 & 862 & 20 & 22 & 97 & 100 & 1 \\
\hline NCGC00160398, $10 \mu \mathrm{M}$ & 927 & 953 & 67 & 72 & 103 & 105 & 0 \\
\hline NCGC00025297, $10 \mu \mathrm{M}$ & 854 & 856 & 23 & 23 & 99 & 99 & 1 \\
\hline Ub-Aldehyde, $0.01 \mu \mathrm{M}$ & 847 & 845 & 26 & 21 & 98 & 98 & 2 \\
\hline Ub-Aldehyde, $0.1 \mu \mathrm{M}$ & 388 & 391 & 23 & 24 & 43 & 43 & 57 \\
\hline Ub-Aldehyde, $1 \mu \mathrm{M}$ & 58 & 58 & 28 & 29 & 3 & 3 & 97 \\
\hline Background & 29 & 13 & 14 & 14 & & & \\
\hline
\end{tabular}

USP5 Activity

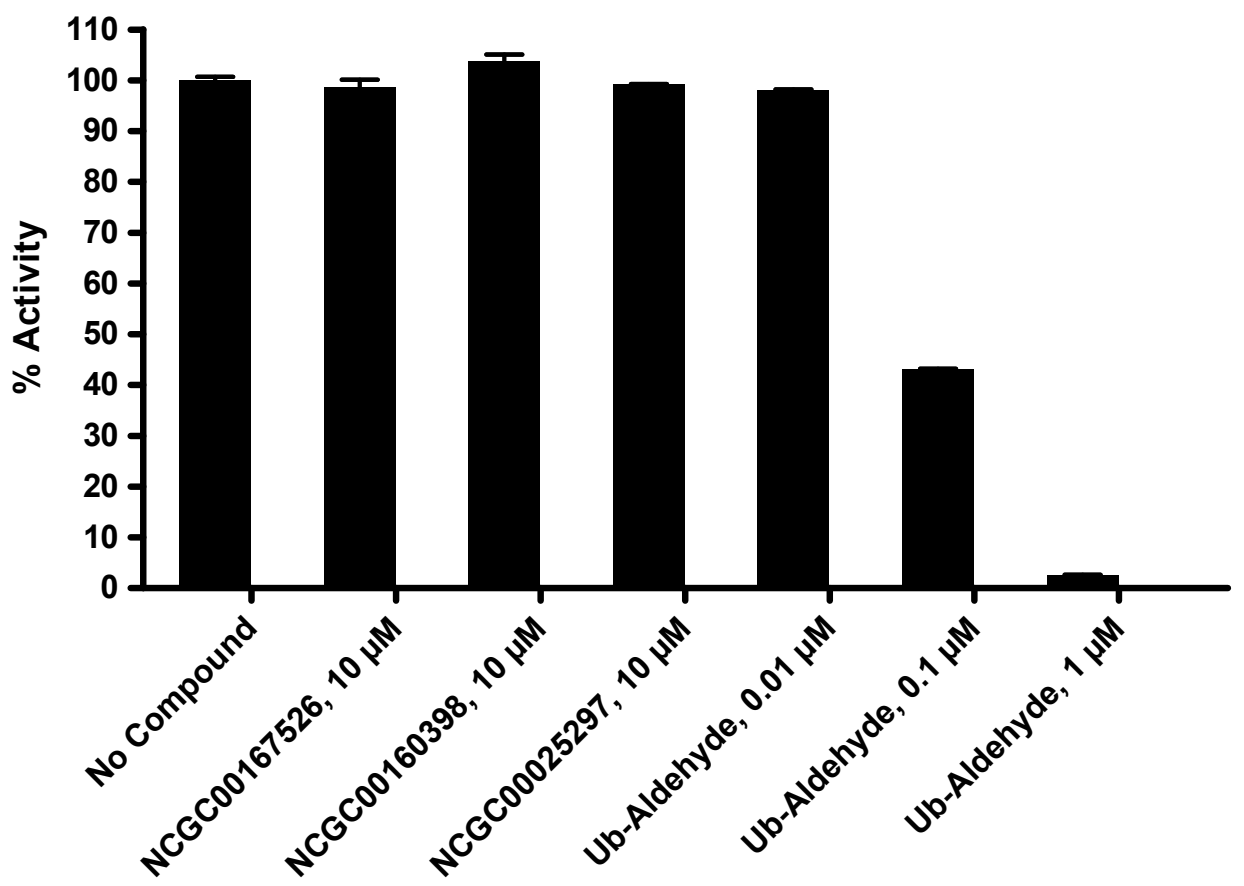


6042 Cornerstone Court West, Suite B

San Diego, CA 92121

Tel: 1.858 .829 .3082

Fax: 1.858 .481 .8694

Email: info@bpsbioscience.com

\subsubsection{USP7}

Table 3.2.22. Data for the Effect of the Compounds on USP7 Activity

\begin{tabular}{|c|c|c|c|c|c|c|c|}
\hline \multirow[t]{2}{*}{ Compounds } & \multicolumn{2}{|c|}{$\begin{array}{c}\text { USP7 Activity } \\
\text { (Fluorescence count) }\end{array}$} & \multicolumn{2}{|c|}{$\begin{array}{c}\text { Background } \\
\text { (Fluorescence count) }\end{array}$} & \multicolumn{2}{|c|}{$\%$ Activity } & \multirow[t]{2}{*}{$\%$ Inhibitior } \\
\hline & Repeat1 & Repeat2 & Repeat1 & Repeat2 & Repeat1 & Repeat2 & \\
\hline No Compound & 524 & 523 & 28 & 29 & 100 & 100 & 0 \\
\hline NCGC00167526, $10 \mu \mathrm{M}$ & 535 & 512 & 30 & 27 & 102 & 98 & 0 \\
\hline NCGC00160398, $10 \mu \mathrm{M}$ & 583 & 593 & 73 & 79 & 103 & 104 & 0 \\
\hline NCGC00025297, $10 \mu \mathrm{M}$ & 529 & 522 & 29 & 29 & 101 & 100 & 0 \\
\hline Ub-Aldehyde, $0.001 \mu \mathrm{M}$ & 460 & 466 & 27 & 28 & 87 & 88 & 12 \\
\hline Ub-Aldehyde, $0.01 \mu \mathrm{M}$ & 250 & 243 & 28 & 26 & 45 & 44 & 56 \\
\hline Ub-Aldehyde, $0.1 \mu \mathrm{M}$ & 40 & 41 & 23 & 24 & 3 & 3 & 97 \\
\hline Background & 13 & 11 & 12 & 10 & & & \\
\hline
\end{tabular}

USP7 Activity

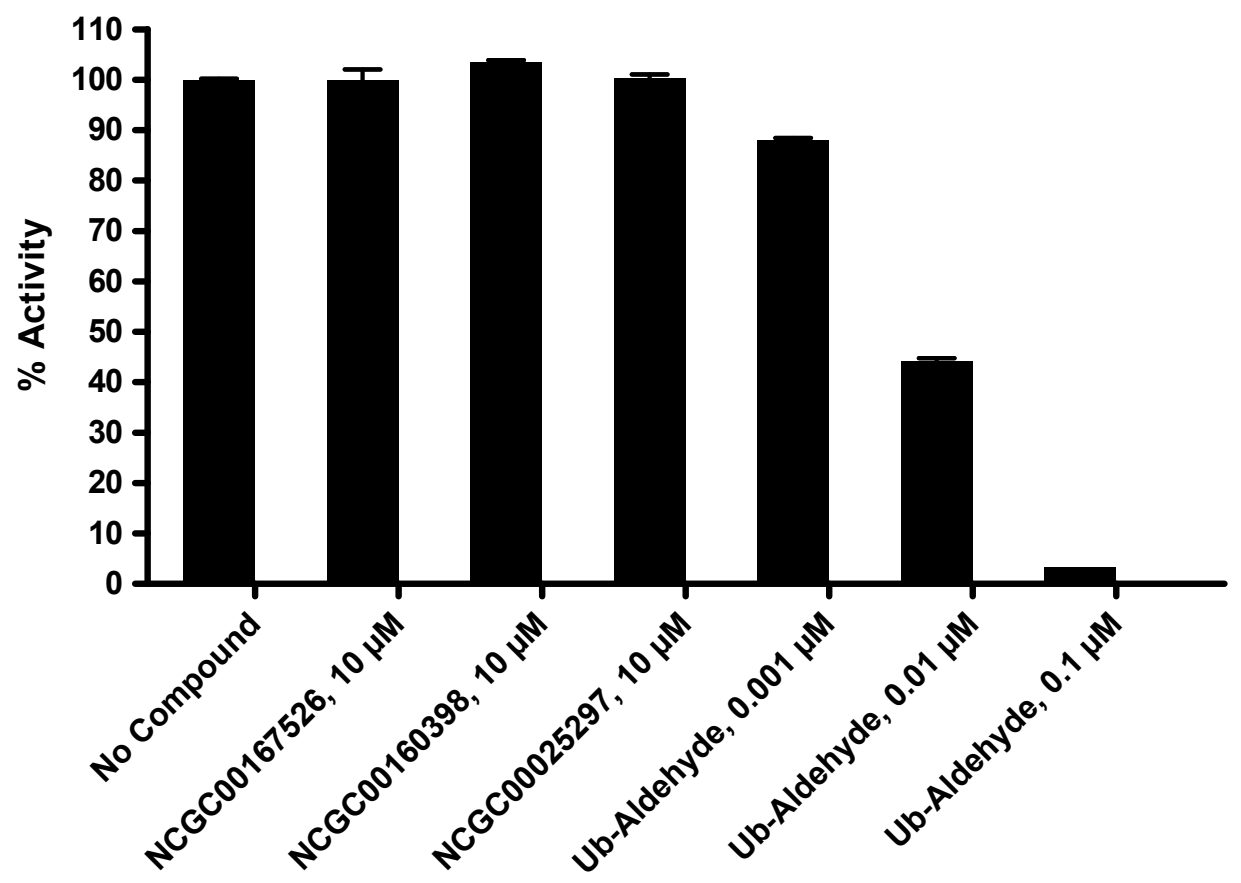


6042 Cornerstone Court West, Suite B

San Diego, CA 92121

Tel: 1.858 .829 .3082

Fax: 1.858 .481 .8694

Email: info@bpsbioscience.com

\subsubsection{USP8}

Table 3.2.23. Data for the Effect of the Compounds on USP8 Activity

\begin{tabular}{|c|c|c|c|c|c|c|c|}
\hline \multirow[t]{2}{*}{ Compounds } & \multicolumn{2}{|c|}{$\begin{array}{c}\text { USP8 Activity } \\
\text { (Fluorescence count) }\end{array}$} & \multicolumn{2}{|c|}{$\begin{array}{c}\text { Background } \\
\text { (Fluorescence count) }\end{array}$} & \multicolumn{2}{|c|}{$\%$ Activity } & \multirow[t]{2}{*}{$\%$ Inhibitior } \\
\hline & Repeat1 & Repeat2 & Repeat1 & Repeat2 & Repeat1 & Repeat2 & \\
\hline No Compound & 210 & 213 & 19 & 20 & 99 & 101 & 0 \\
\hline NCGC00167526, $10 \mu \mathrm{M}$ & 220 & 213 & 20 & 19 & 104 & 101 & 0 \\
\hline NCGC00160398, $10 \mu \mathrm{M}$ & 247 & 254 & 64 & 71 & 95 & 95 & 5 \\
\hline NCGC00025297, $10 \mu \mathrm{M}$ & 212 & 219 & 20 & 20 & 100 & 104 & 0 \\
\hline Ub-Aldehyde, $0.01 \mu \mathrm{M}$ & 192 & 188 & 19 & 19 & 90 & 88 & 11 \\
\hline Ub-Aldehyde, $0.1 \mu \mathrm{M}$ & 143 & 143 & 18 & 19 & 65 & 65 & 35 \\
\hline Ub-Aldehyde, $1 \mu \mathrm{M}$ & 47 & 47 & 18 & 17 & 15 & 16 & 85 \\
\hline Background & 13 & 13 & 13 & 13 & & & \\
\hline
\end{tabular}

USP8 Activity

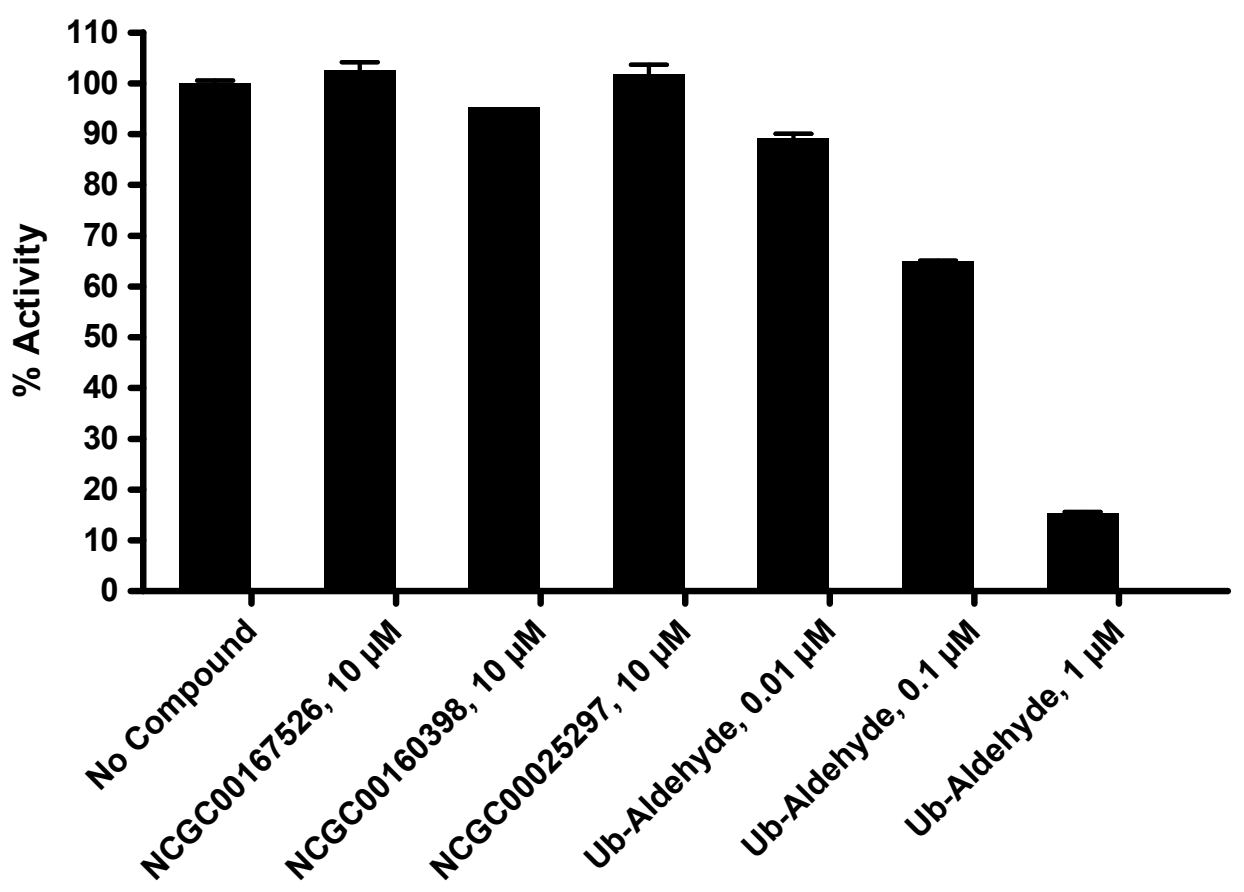


6042 Cornerstone Court West, Suite B

San Diego, CA 92121

Tel: 1.858 .829 .3082

Fax: 1.858 .481 .8694

Email: info@bpsbioscience.com

\subsubsection{USP10}

Table 3.2.24. Data for the Effect of the Compounds on USP10 Activity

\begin{tabular}{|c|c|c|c|c|c|c|c|}
\hline \multirow[t]{2}{*}{ Compounds } & \multicolumn{2}{|c|}{$\begin{array}{l}\text { USP10 Activity } \\
\text { (Fluorescence count) }\end{array}$} & \multicolumn{2}{|c|}{$\begin{array}{c}\text { Background } \\
\text { (Fluorescence count) }\end{array}$} & \multicolumn{2}{|c|}{$\%$ Activity } & \multirow[t]{2}{*}{$\%$ Inhibition } \\
\hline & Repeat1 & Repeat2 & Repeat1 & Repeat2 & Repeat1 & Repeat2 & \\
\hline No Compound & 331 & 353 & 28 & 33 & 97 & 103 & 0 \\
\hline NCGC00167526, $10 \mu \mathrm{M}$ & 321 & 349 & 28 & 31 & 94 & 102 & 2 \\
\hline NCGC00160398, $10 \mu \mathrm{M}$ & 395 & 399 & 77 & 86 & 102 & 100 & 0 \\
\hline NCGC00025297, $10 \mu \mathrm{M}$ & 325 & 347 & 31 & 34 & 94 & 100 & 3 \\
\hline Ub-Aldehyde, $0.01 \mu \mathrm{M}$ & 327 & 325 & 35 & 27 & 94 & 96 & 5 \\
\hline Ub-Aldehyde, $0.1 \mu \mathrm{M}$ & 207 & 217 & 28 & 34 & 57 & 58 & 43 \\
\hline Ub-Aldehyde, $1 \mu \mathrm{M}$ & 86 & 97 & 29 & 41 & 17 & 17 & 83 \\
\hline Background & 9 & 9 & 14 & 14 & & & \\
\hline
\end{tabular}

USP10 Activity

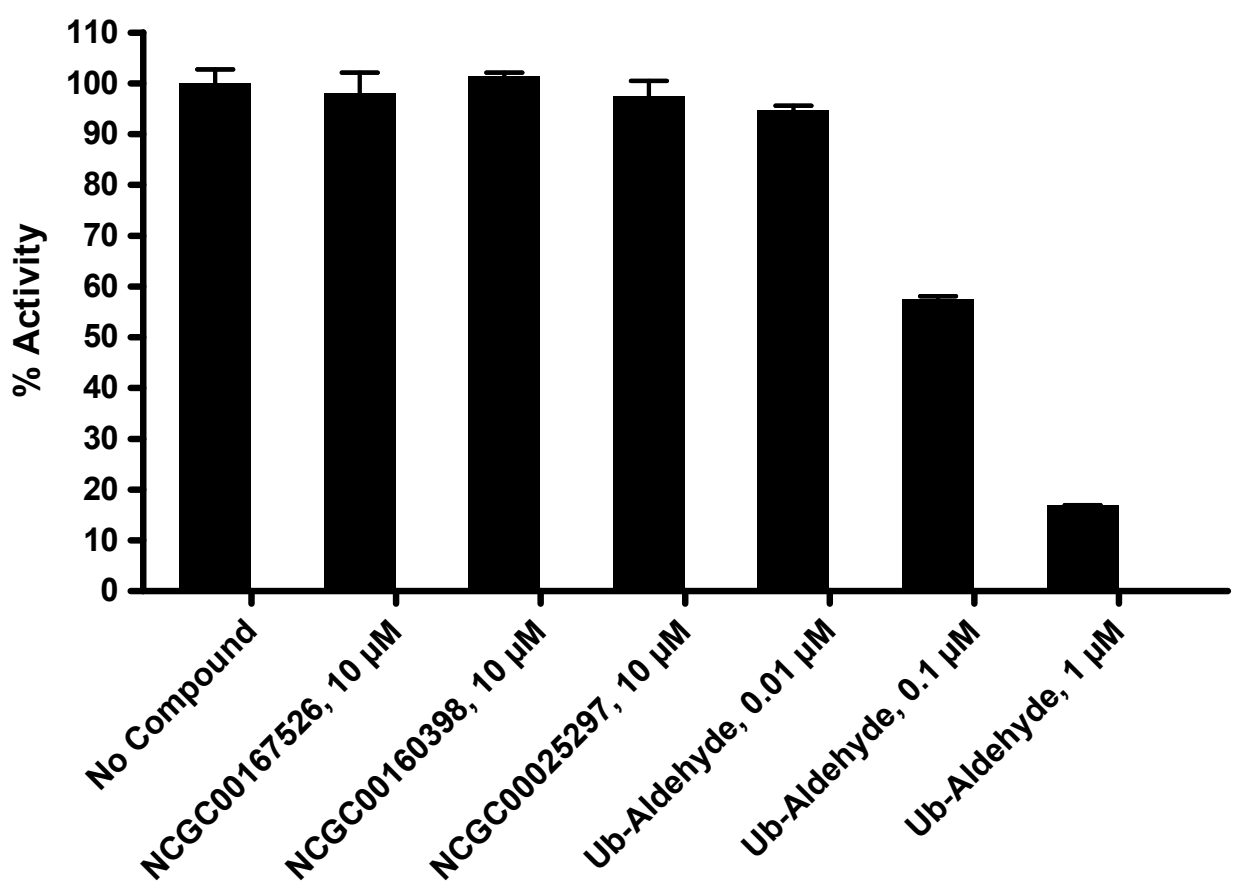


6042 Cornerstone Court West, Suite B

San Diego, CA 92121

Tel: 1.858 .829 .3082

Fax: 1.858 .481 .8694

Email: info@bpsbioscience.com

\subsubsection{USP14}

Table 3.2.25. Data for the Effect of the Compounds on USP14 Activity

\begin{tabular}{|c|c|c|c|c|c|c|c|}
\hline \multirow[t]{2}{*}{ Compounds } & \multicolumn{2}{|c|}{$\begin{array}{l}\text { USP14 Activity } \\
\text { (Fluorescence count) }\end{array}$} & \multicolumn{2}{|c|}{$\begin{array}{c}\text { Background } \\
\text { (Fluorescence count) }\end{array}$} & \multicolumn{2}{|c|}{$\%$ Activity } & \multirow[t]{2}{*}{$\%$ Inhibition } \\
\hline & Repeat1 & Repeat2 & Repeat1 & Repeat2 & Repeat1 & Repeat2 & \\
\hline No Compound & 57 & 66 & 17 & 18 & 90 & 110 & 0 \\
\hline NCGC00167526, $10 \mu \mathrm{M}$ & 58 & 66 & 16 & 17 & 95 & 113 & 0 \\
\hline NCGC00160398, $10 \mu \mathrm{M}$ & 139 & 136 & 62 & 57 & 184 & 189 & 0 \\
\hline NCGC00025297, $10 \mu \mathrm{M}$ & 58 & 65 & 18 & 18 & 90 & 108 & 1 \\
\hline Ub-Aldehyde, $0.001 \mu \mathrm{M}$ & 57 & 52 & 19 & 17 & 85 & 77 & 19 \\
\hline Ub-Aldehyde, $0.01 \mu \mathrm{M}$ & 34 & 32 & 18 & 17 & 29 & 27 & 72 \\
\hline Ub-Aldehyde, $0.1 \mu \mathrm{M}$ & 17 & 18 & 17 & 18 & 0 & 0 & 100 \\
\hline Background & 11 & 11 & 16 & 15 & & & \\
\hline
\end{tabular}

USP14 Activity

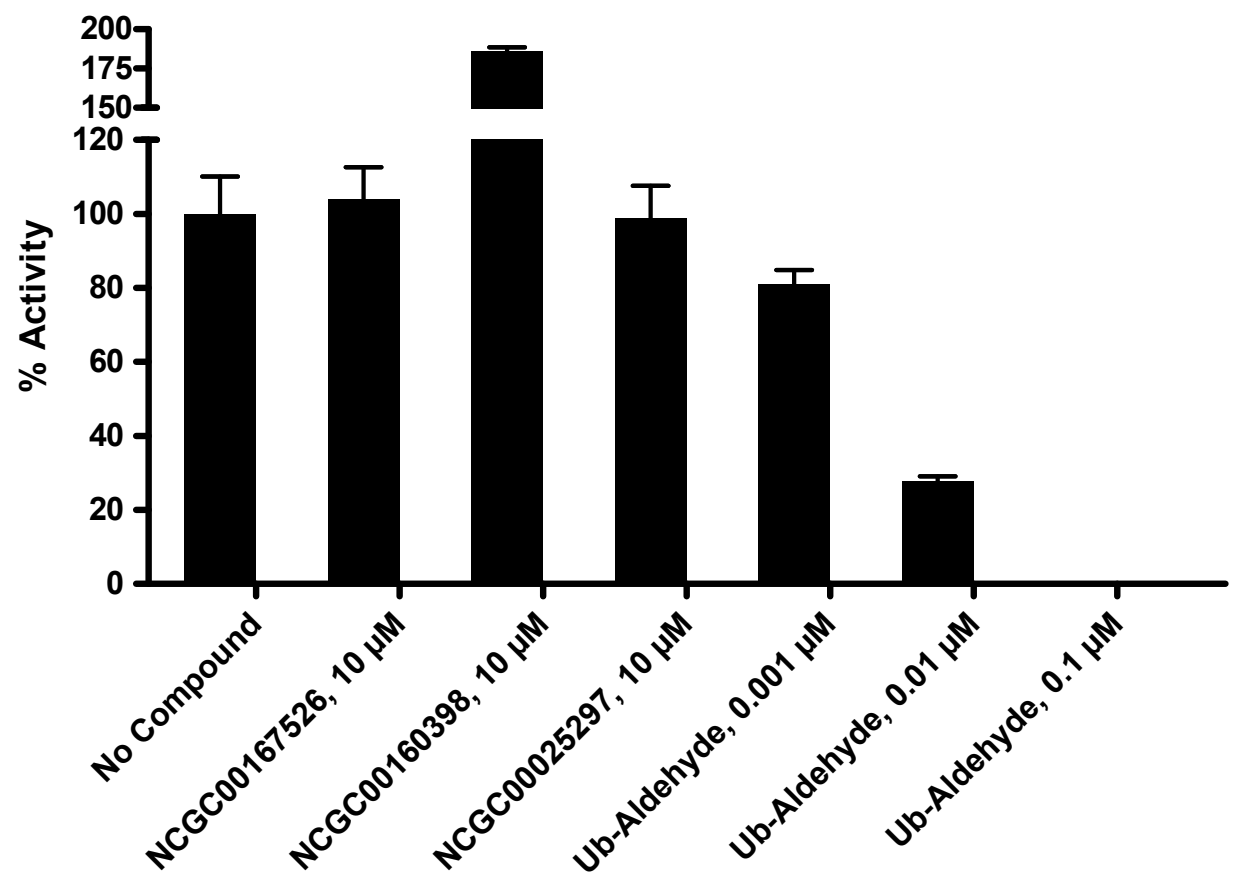


6042 Cornerstone Court West, Suite B

San Diego, CA 92121

Tel: 1.858 .829 .3082

Fax: 1.858 .481 .8694

Email: info@bpsbioscience.com

\subsubsection{ADAM17}

Table 3.2.26. Data for the Effect of the Compounds on ADAM17 Activity

\begin{tabular}{|c|c|c|c|c|c|c|c|}
\hline \multirow{2}{*}{ Compounds } & \multicolumn{2}{|c|}{$\begin{array}{l}\text { ADAM17 Activity } \\
\text { (Fluorescence count) }\end{array}$} & \multicolumn{2}{|c|}{$\begin{array}{c}\text { Background } \\
\text { (Fluorescence count) }\end{array}$} & \multicolumn{2}{|c|}{$\%$ Activity } & \multirow[t]{2}{*}{$\%$ Inhibition } \\
\hline & Repeat1 & Repeat2 & Repeat1 & Repeat2 & Repeat1 & Repeat2 & \\
\hline No Compound & 42801 & 42329 & 6838 & 6676 & 100 & 100 & 0 \\
\hline NCGC00167526, $10 \mu \mathrm{M}$ & 40520 & 41117 & 7128 & 7511 & 93 & 94 & 6 \\
\hline NCGC00160398, $10 \mu \mathrm{M}$ & 40625 & 41349 & 6852 & 7156 & 94 & 95 & 5 \\
\hline NCGC00025297, $10 \mu \mathrm{M}$ & 40489 & 39926 & 6784 & 6885 & 94 & 92 & 7 \\
\hline TAPI-2, $0.1 \mu \mathrm{M}$ & 42733 & 42649 & 7037 & 6796 & 100 & 100 & 0 \\
\hline TAPI- $2,1 \mu \mathrm{M}$ & 39241 & 36415 & 6823 & 6600 & 90 & 83 & 13 \\
\hline TAPI-2, $10 \mu \mathrm{M}$ & 17326 & 15501 & 6635 & 6396 & 30 & 25 & 73 \\
\hline Background & 5504 & 5482 & 5308 & 5350 & & & \\
\hline
\end{tabular}

ADAM17 Activity

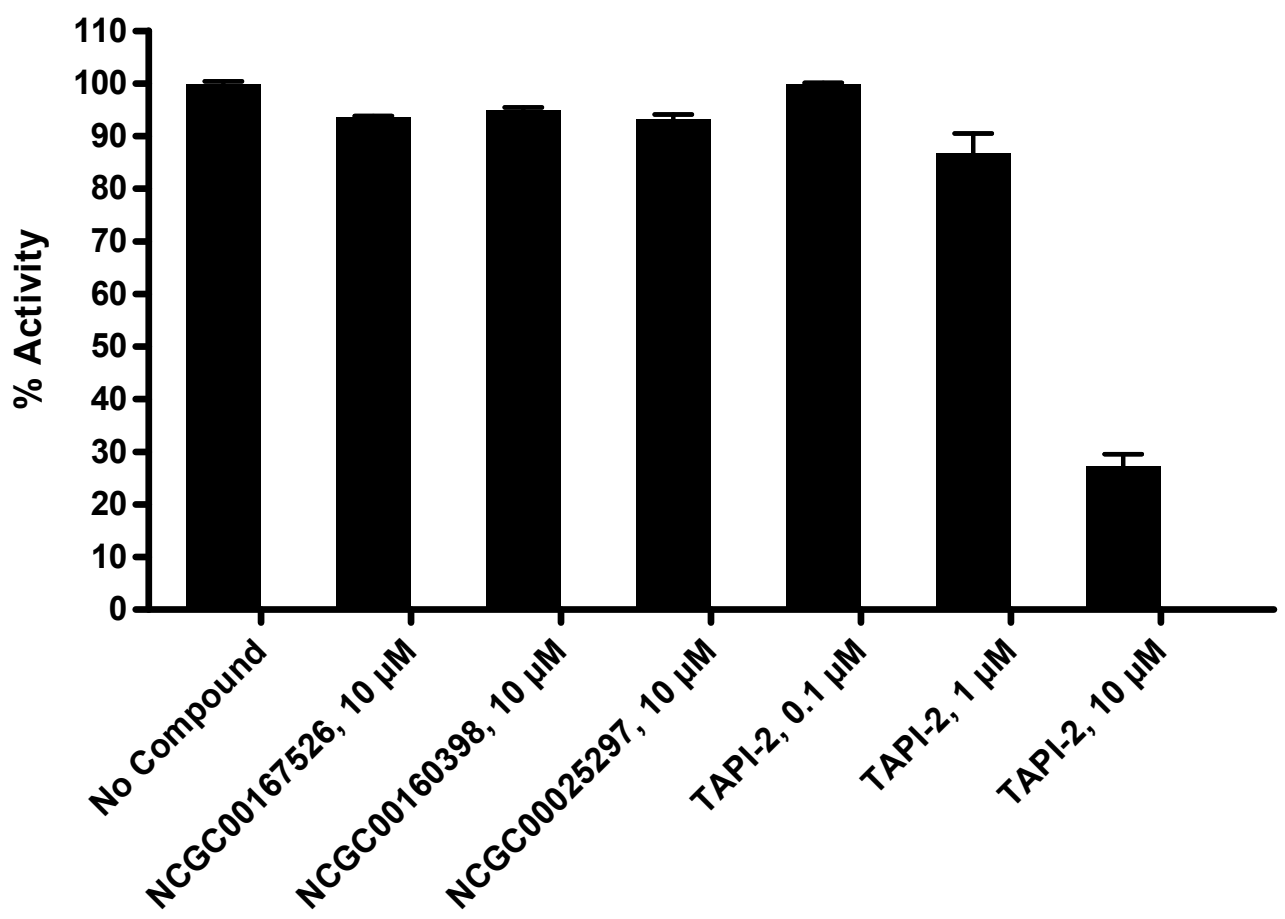


6042 Cornerstone Court West, Suite B

San Diego, CA 92121

Tel: 1.858 .829 .3082

Fax: 1.858 .481 .8694

Email: info@bpsbioscience.com

\subsubsection{MMP1}

Table 3.2.27. Data for the Effect of the Compounds on MMP1 Activity

\begin{tabular}{|c|c|c|c|c|c|c|c|}
\hline \multirow[t]{2}{*}{ Compounds } & \multicolumn{2}{|c|}{$\begin{array}{l}\text { MMP1 Activity } \\
\text { (Fluorescence count) }\end{array}$} & \multicolumn{2}{|c|}{$\begin{array}{c}\text { Background } \\
\text { (Fluorescence count) }\end{array}$} & \multicolumn{2}{|c|}{$\%$ Activity } & \multirow[t]{2}{*}{$\%$ Inhibition } \\
\hline & Repeat1 & Repeat2 & Repeat1 & Repeat2 & Repeat1 & Repeat2 & \\
\hline No Compound & 2709 & 2726 & 587 & 591 & 100 & 100 & 0 \\
\hline NCGC00167526, $10 \mu \mathrm{M}$ & 2638 & 2631 & 569 & 564 & 97 & 97 & 3 \\
\hline NCGC00160398, $10 \mu \mathrm{M}$ & 3274 & 3220 & 1040 & 1015 & 105 & 104 & 0 \\
\hline NCGC00025297, $10 \mu \mathrm{M}$ & 2649 & 2660 & 560 & 562 & 98 & 99 & 2 \\
\hline NNGH, $0.1 \mu \mathrm{M}$ & 2639 & 2645 & 548 & 548 & 98 & 98 & 2 \\
\hline $\mathrm{NNGH}, 1 \mu \mathrm{M}$ & 2138 & 2130 & 558 & 550 & 74 & 74 & 26 \\
\hline $\mathrm{NNGH}, 10 \mu \mathrm{M}$ & 1066 & 1039 & 537 & 511 & 23 & 23 & 77 \\
\hline Background & 533 & 536 & 486 & 491 & & & \\
\hline
\end{tabular}

\section{MMP1 Activity}

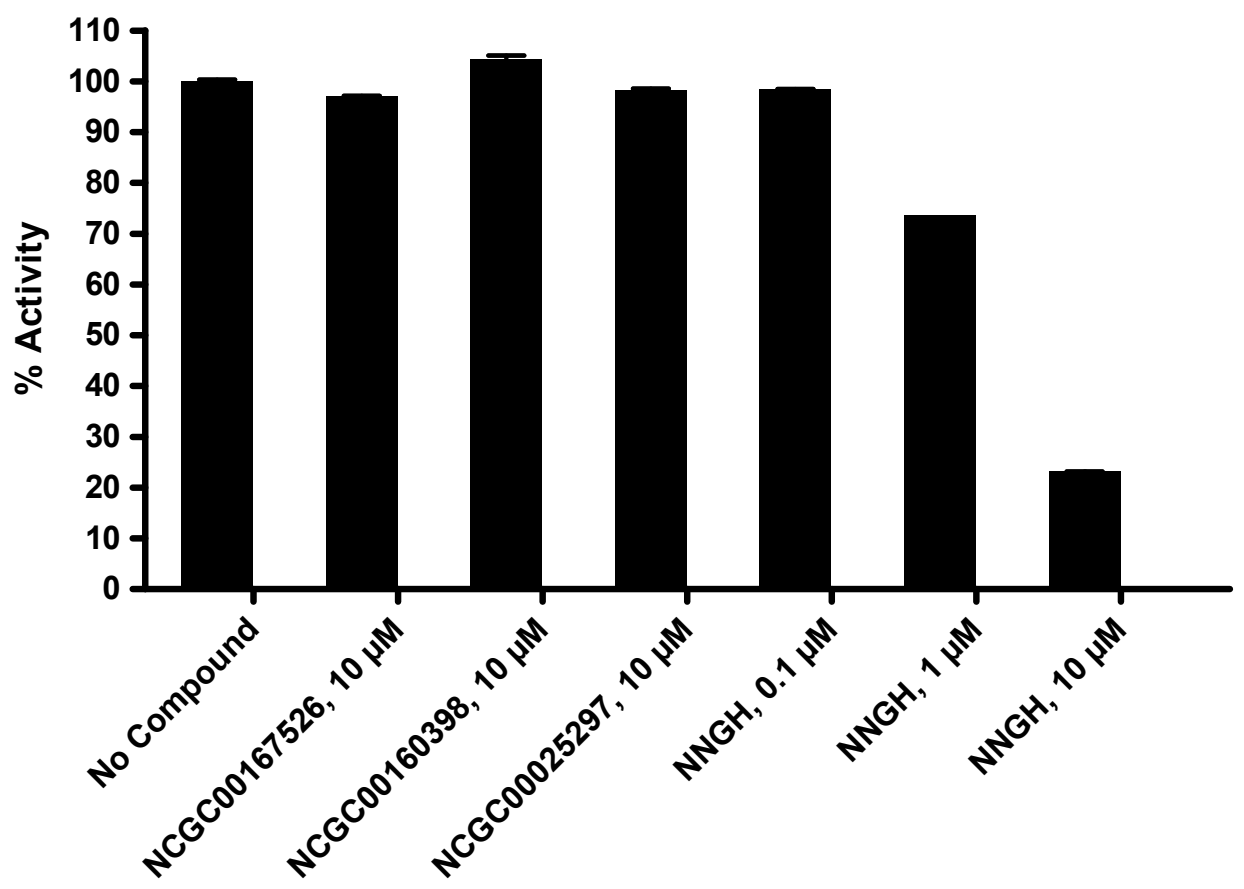


6042 Cornerstone Court West, Suite B

San Diego, CA 92121

Tel: 1.858 .829 .3082

Fax: 1.858 .481 .8694

Email: info@bpsbioscience.com

3.2.28. MMP2

Table 3.2.28. Data for the Effect of the Compounds on MMP2 Activity

\begin{tabular}{|c|c|c|c|c|c|c|c|}
\hline \multirow[t]{2}{*}{ Compounds } & \multicolumn{2}{|c|}{$\begin{array}{l}\text { MMP2 Activity } \\
\text { (Fluorescence count) }\end{array}$} & \multicolumn{2}{|c|}{$\begin{array}{c}\text { Background } \\
\text { (Fluorescence count) }\end{array}$} & \multicolumn{2}{|c|}{$\%$ Activity } & \multirow[t]{2}{*}{$\%$ Inhibition } \\
\hline & Repeat1 & Repeat2 & Repeat1 & Repeat2 & Repeat1 & Repeat2 & \\
\hline No Compound & 337 & 335 & 158 & 158 & 101 & 99 & 0 \\
\hline NCGC00167526, $10 \mu \mathrm{M}$ & 210 & 198 & 160 & 161 & 21 & 13 & 83 \\
\hline NCGC00160398, $10 \mu \mathrm{M}$ & 985 & 997 & 627 & 618 & 211 & 224 & 0 \\
\hline NCGC00025297, $10 \mu \mathrm{M}$ & 303 & 286 & 166 & 153 & 75 & 72 & 26 \\
\hline $\mathrm{NNGH}, 0.01 \mu \mathrm{M}$ & 290 & 285 & 154 & 143 & 74 & 78 & 24 \\
\hline $\mathrm{NNGH}, 0.1 \mu \mathrm{M}$ & 207 & 205 & 143 & 144 & 30 & 28 & 71 \\
\hline $\mathrm{NNGH}, 1 \mu \mathrm{M}$ & 160 & 160 & 151 & 146 & 0 & 0 & 100 \\
\hline Background & 163 & 163 & 147 & 148 & & & \\
\hline
\end{tabular}

\section{MMP2 Activity}

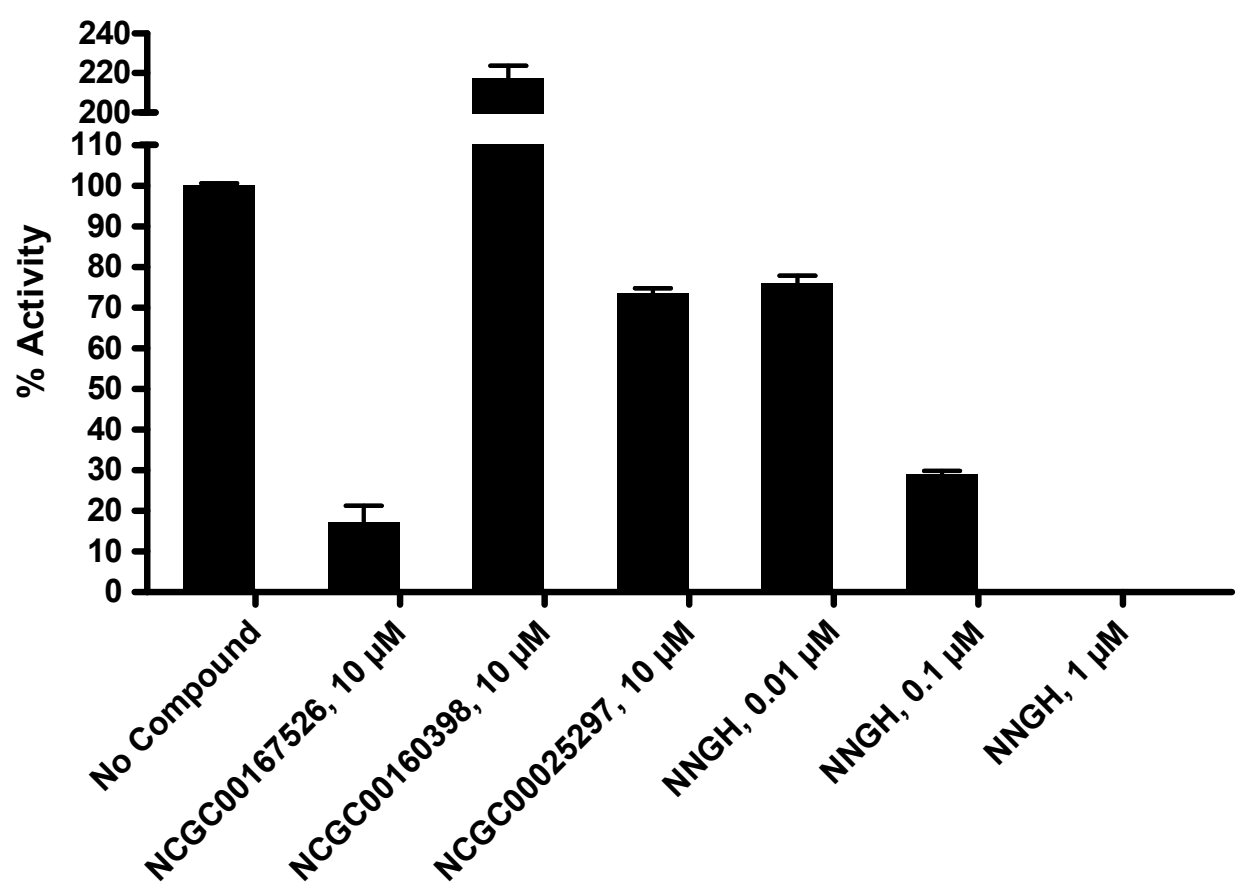


6042 Cornerstone Court West, Suite B

San Diego, CA 92121

Tel: 1.858 .829 .3082

Fax: 1.858 .481 .8694

Email: info@bpsbioscience.com

\subsubsection{MMP3}

Table 3.2.29. Data for the Effect of the Compounds on MMP3 Activity

\begin{tabular}{|c|c|c|c|c|c|c|c|}
\hline \multirow[t]{2}{*}{ Compounds } & \multicolumn{2}{|c|}{$\begin{array}{l}\text { MMP3 Activity } \\
\text { (Fluorescence count) }\end{array}$} & \multicolumn{2}{|c|}{$\begin{array}{c}\text { Background } \\
\text { (Fluorescence count) }\end{array}$} & \multicolumn{2}{|c|}{$\%$ Activity } & \multirow[t]{2}{*}{$\%$ Inhibition } \\
\hline & Repeat1 & Repeat2 & Repeat1 & Repeat2 & Repeat1 & Repeat2 & \\
\hline No Compound & 470 & 480 & 258 & 268 & 100 & 100 & 0 \\
\hline NCGC00167526, $10 \mu \mathrm{M}$ & 421 & 422 & 266 & 265 & 73 & 74 & 27 \\
\hline NCGC00160398, $10 \mu \mathrm{M}$ & 1054 & 1028 & 688 & 684 & 173 & 163 & 0 \\
\hline NCGC00025297, $10 \mu \mathrm{M}$ & 417 & 428 & 245 & 253 & 81 & 82 & 18 \\
\hline $\mathrm{NNGH}, 0.01 \mu \mathrm{M}$ & 426 & 422 & 256 & 253 & 80 & 80 & 20 \\
\hline $\mathrm{NNGH}, 0.1 \mu \mathrm{M}$ & 298 & 298 & 255 & 253 & 20 & 20 & 80 \\
\hline $\mathrm{NNGH}, 1 \mu \mathrm{M}$ & 242 & 241 & 242 & 245 & 0 & 1 & 100 \\
\hline Background & 144 & 149 & 144 & 145 & & & \\
\hline
\end{tabular}

\section{MMP3 Activity}

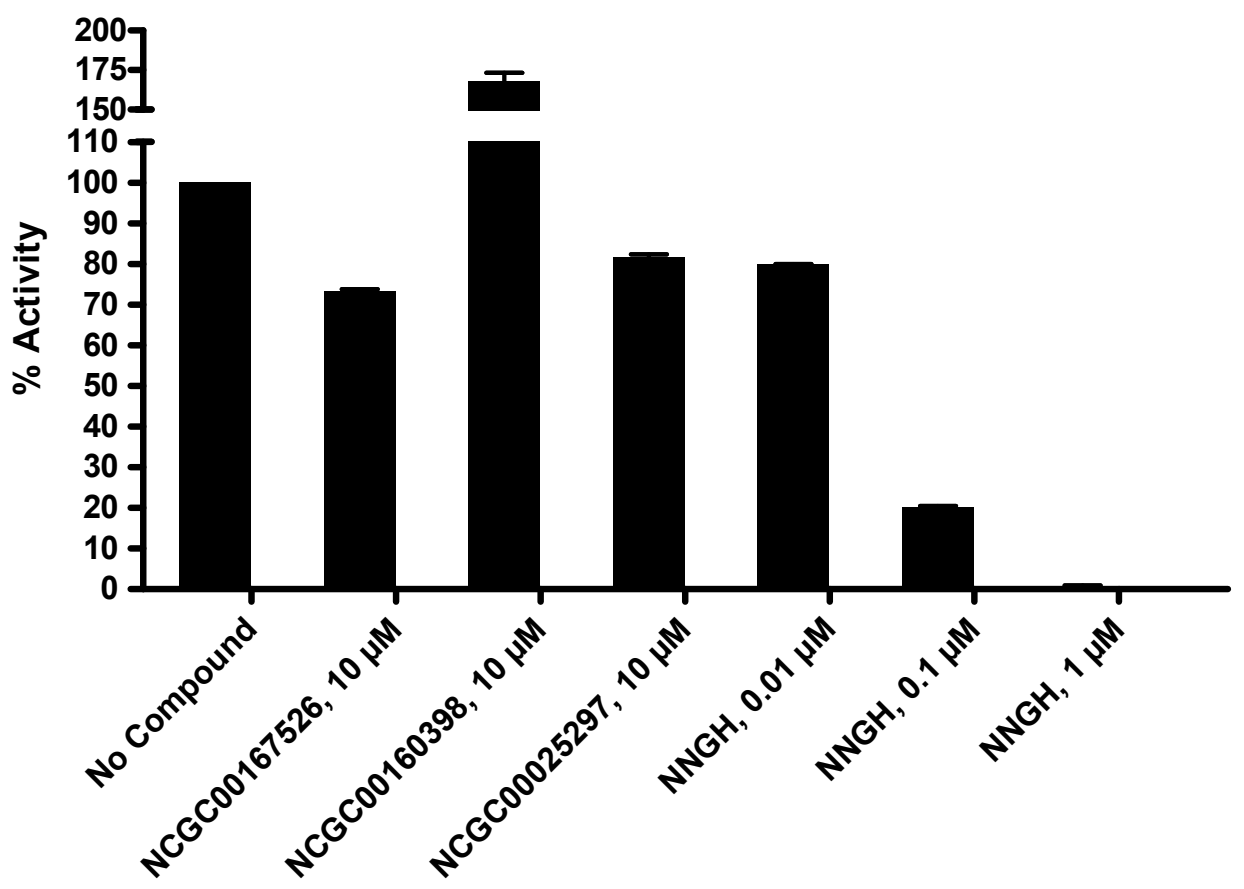


6042 Cornerstone Court West, Suite B

San Diego, CA 92121

Tel: 1.858 .829 .3082

Fax: 1.858 .481 .8694

Email: info@bpsbioscience.com

\subsubsection{MMP7}

Table 3.2.30. Data for the Effect of the Compounds on MMP7 Activity

\begin{tabular}{|c|c|c|c|c|c|c|c|}
\hline \multirow[t]{2}{*}{ Compounds } & \multicolumn{2}{|c|}{$\begin{array}{l}\text { MMP7 Activity } \\
\text { (Fluorescence count) }\end{array}$} & \multicolumn{2}{|c|}{$\begin{array}{c}\text { Background } \\
\text { (Fluorescence count) }\end{array}$} & \multicolumn{2}{|c|}{$\%$ Activity } & \multirow[t]{2}{*}{$\%$ Inhibition } \\
\hline & Repeat1 & Repeat2 & Repeat1 & Repeat2 & Repeat1 & Repeat2 & \\
\hline No Compound & 13151 & 13336 & 721 & 821 & 100 & 100 & 0 \\
\hline NCGC00167526, $10 \mu \mathrm{M}$ & 13024 & 12955 & 731 & 811 & 99 & 97 & 2 \\
\hline NCGC00160398, $10 \mu \mathrm{M}$ & 13050 & 13055 & 1193 & 1214 & 95 & 95 & 5 \\
\hline NCGC00025297, $10 \mu \mathrm{M}$ & 12727 & 12789 & 661 & 651 & 97 & 97 & 3 \\
\hline Batimastat, $0.0001 \mu \mathrm{M}$ & 12628 & 12882 & 792 & 827 & 95 & 97 & 4 \\
\hline Batimastat, $0.001 \mu \mathrm{M}$ & 9330 & 9338 & 707 & 679 & 69 & 69 & 31 \\
\hline Batimastat, $0.01 \mu \mathrm{M}$ & 1369 & 1398 & 587 & 624 & 6 & 6 & 94 \\
\hline Background & 161 & 159 & 138 & 135 & & & \\
\hline
\end{tabular}

MMP7 Activity

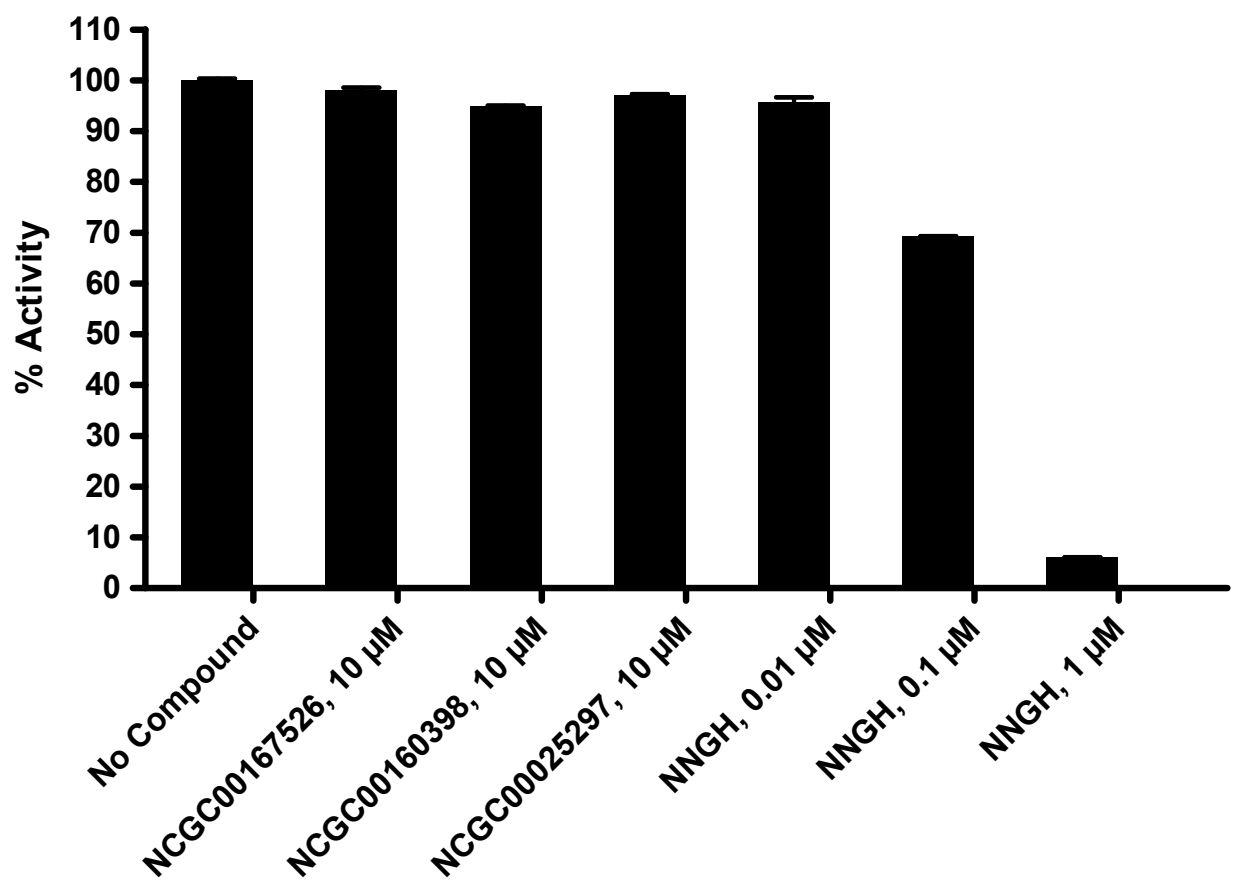


6042 Cornerstone Court West, Suite B

San Diego, CA 92121

Tel: 1.858 .829 .3082

Fax: 1.858 .481 .8694

Email: info@bpsbioscience.com

\subsubsection{MMP8}

Table 3.2.31. Data for the Effect of the Compounds on MMP8 Activity

\begin{tabular}{|c|c|c|c|c|c|c|c|}
\hline \multirow[t]{2}{*}{ Compounds } & \multicolumn{2}{|c|}{$\begin{array}{l}\text { MMP8 Activity } \\
\text { (Fluorescence count) }\end{array}$} & \multicolumn{2}{|c|}{$\begin{array}{c}\text { Background } \\
\text { (Fluorescence count) }\end{array}$} & \multicolumn{2}{|c|}{$\%$ Activity } & \multirow[t]{2}{*}{$\%$ Inhibition } \\
\hline & Repeat1 & Repeat2 & Repeat1 & Repeat2 & Repeat1 & Repeat2 & \\
\hline No Compound & 268 & 264 & 92 & 86 & 99 & 101 & 0 \\
\hline NCGC00167526, $10 \mu \mathrm{M}$ & 216 & 214 & 99 & 91 & 66 & 69 & 33 \\
\hline NCGC00160398, $10 \mu \mathrm{M}$ & 951 & 952 & 510 & 514 & 251 & 249 & 0 \\
\hline NCGC00025297, $10 \mu \mathrm{M}$ & 247 & 254 & 83 & 89 & 93 & 93 & 7 \\
\hline $\mathrm{NNGH}, 0.01 \mu \mathrm{M}$ & 256 & 251 & 90 & 81 & 94 & 96 & 5 \\
\hline $\mathrm{NNGH}, 0.1 \mu \mathrm{M}$ & 189 & 187 & 88 & 83 & 57 & 58 & 43 \\
\hline $\mathrm{NNGH}, 1 \mu \mathrm{M}$ & 101 & 105 & 82 & 87 & 10 & 9 & 91 \\
\hline Background & 85 & 80 & 87 & 82 & & & \\
\hline
\end{tabular}

\section{MMP8 Activity}

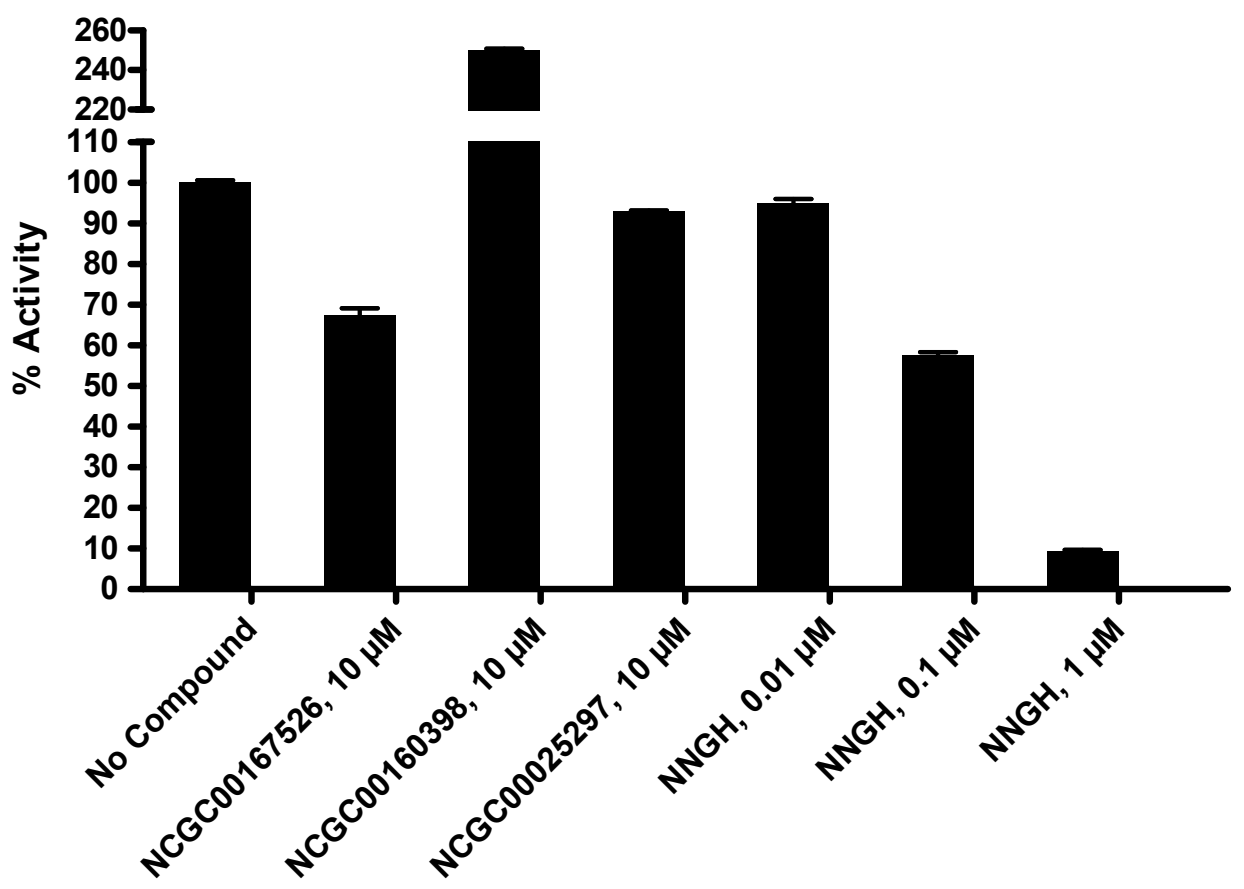


6042 Cornerstone Court West, Suite B

San Diego, CA 92121

Tel: 1.858 .829 .3082

Fax: 1.858 .481 .8694

Email: info@bpsbioscience.com

3.2.32. MMP9 (O279R)

Table 3.2.32. Data for the Effect of the Compounds on MMP9 (Q279R) Activity

\begin{tabular}{|c|c|c|c|c|c|c|c|}
\hline \multirow[t]{2}{*}{ Compounds } & \multicolumn{2}{|c|}{$\begin{array}{c}\text { MMP9 (Q279R) } \\
\text { Activity } \\
\text { (Fluorescence count) }\end{array}$} & \multicolumn{2}{|c|}{$\begin{array}{c}\text { Background } \\
\text { (Fluorescence count) }\end{array}$} & \multicolumn{2}{|c|}{$\%$ Activity } & \multirow[t]{2}{*}{$\%$ Inhibition } \\
\hline & Repeat1 & Repeat2 & Repeat1 & Repeat2 & Repeat1 & Repeat2 & \\
\hline No Compound & 731 & 718 & 93 & 85 & 100 & 100 & 0 \\
\hline NCGC00167526, $10 \mu \mathrm{M}$ & 682 & 673 & 93 & 87 & 93 & 92 & 8 \\
\hline NCGC00160398, $10 \mu \mathrm{M}$ & 854 & 868 & 286 & 306 & 89 & 88 & 11 \\
\hline NCGC00025297, $10 \mu \mathrm{M}$ & 639 & 648 & 84 & 93 & 87 & 87 & 13 \\
\hline $\mathrm{NNGH}, 0.001 \mu \mathrm{M}$ & 558 & 540 & 85 & 83 & 74 & 72 & 27 \\
\hline $\mathrm{NNGH}, 0.01 \mu \mathrm{M}$ & 245 & 258 & 82 & 85 & 26 & 27 & 74 \\
\hline NNGH, $0.1 \mu \mathrm{M}$ & 110 & 105 & 81 & 79 & 4 & 4 & 96 \\
\hline Background & 84 & 85 & 84 & 84 & & & \\
\hline
\end{tabular}

MMP9 (Q279R) Activity

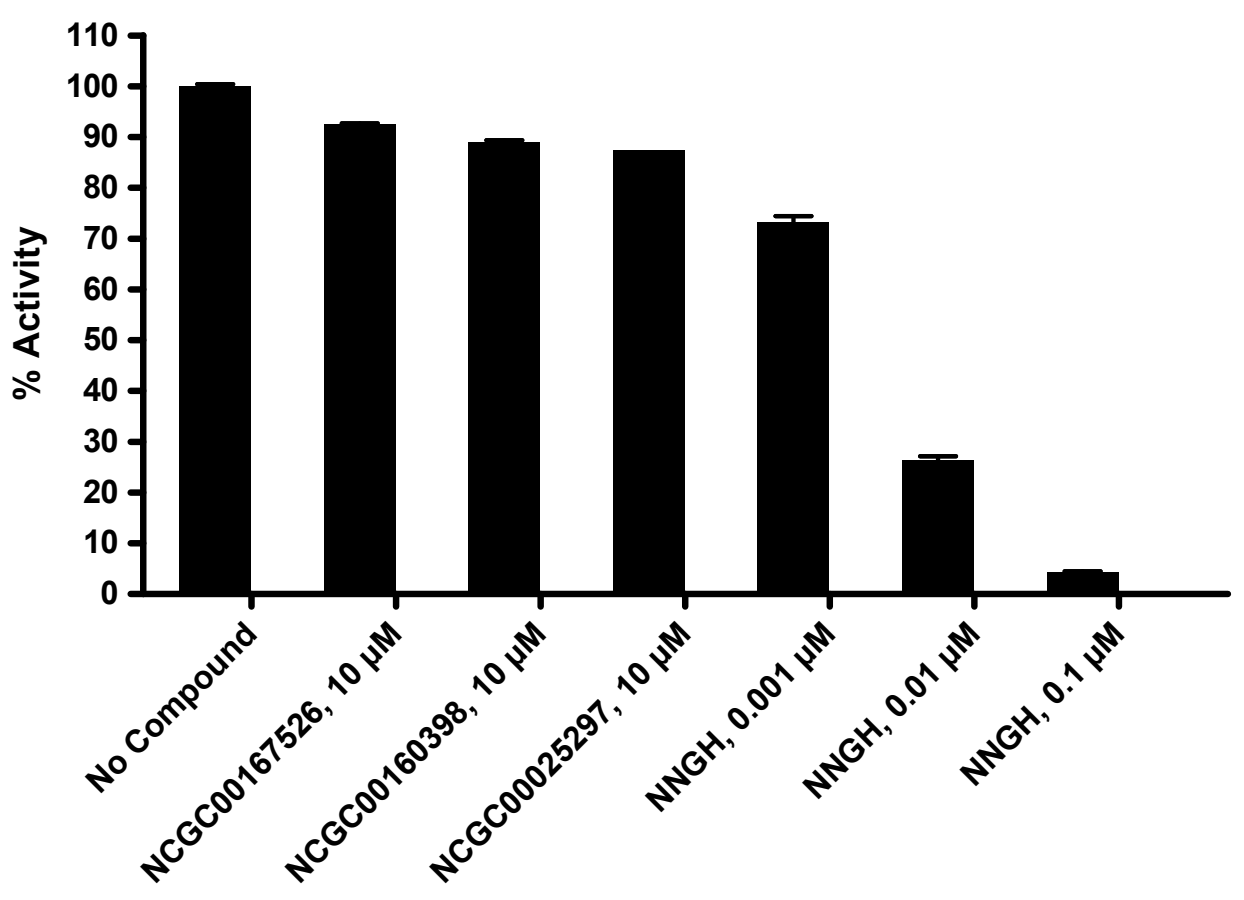


6042 Cornerstone Court West, Suite B

San Diego, CA 92121

Tel: 1.858 .829 .3082

Fax: 1.858 .481 .8694

Email: info@bpsbioscience.com

\subsubsection{MMP10}

Table 3.2.33. Data for the Effect of the Compounds on MMP10 Activity

\begin{tabular}{|c|c|c|c|c|c|c|c|}
\hline \multirow[t]{2}{*}{ Compounds } & \multicolumn{2}{|c|}{$\begin{array}{l}\text { MMP10 Activity } \\
\text { (Fluorescence count) }\end{array}$} & \multicolumn{2}{|c|}{$\begin{array}{c}\text { Background } \\
\text { (Fluorescence count) }\end{array}$} & \multicolumn{2}{|c|}{$\%$ Activity } & \multirow[t]{2}{*}{$\%$ Inhibition } \\
\hline & Repeat1 & Repeat2 & Repeat1 & Repeat2 & Repeat1 & Repeat2 & \\
\hline No Compound & 312 & 318 & 141 & 141 & 98 & 102 & 0 \\
\hline NCGC00167526, $10 \mu \mathrm{M}$ & 266 & 278 & 138 & 156 & 73 & 70 & 29 \\
\hline NCGC00160398, $10 \mu \mathrm{M}$ & 1038 & 1049 & 517 & 551 & 304 & 290 & 0 \\
\hline NCGC00025297, $10 \mu \mathrm{M}$ & 295 & 298 & 140 & 147 & 89 & 87 & 12 \\
\hline NNGH, $0.1 \mu \mathrm{M}$ & 275 & 277 & 141 & 149 & 77 & 73 & 25 \\
\hline $\mathrm{NNGH}, 1 \mu \mathrm{M}$ & 213 & 212 & 142 & 150 & 40 & 34 & 63 \\
\hline $\mathrm{NNGH}, 10 \mu \mathrm{M}$ & 152 & 154 & 132 & 135 & 10 & 9 & 91 \\
\hline Background & 152 & 155 & 148 & 152 & & & \\
\hline
\end{tabular}

MMP10 Activity

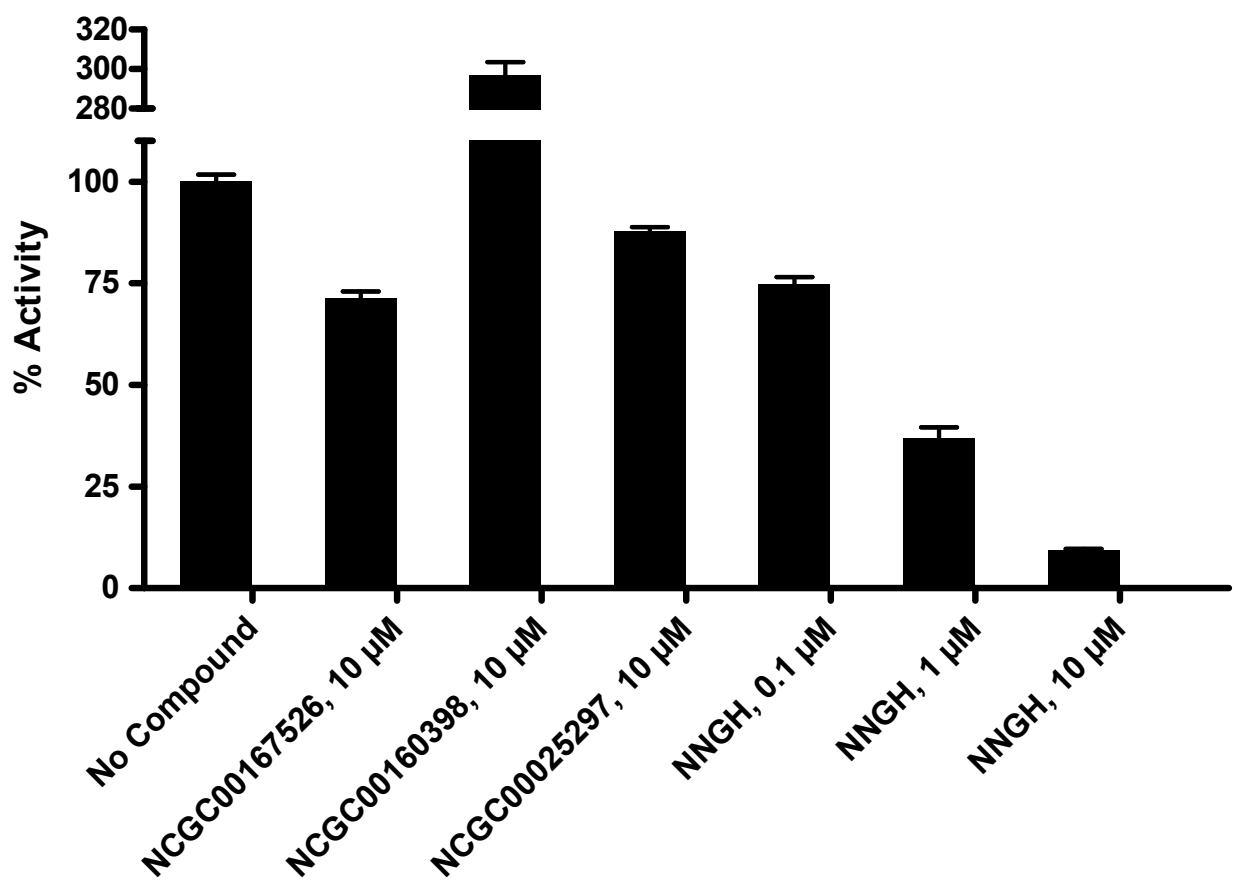


6042 Cornerstone Court West, Suite B

San Diego, CA 92121

Tel: 1.858 .829 .3082

Fax: 1.858 .481 .8694

Email: info@bpsbioscience.com

\subsubsection{MMP13}

Table 3.2.34. Data for the Effect of the Compounds on MMP13 Activity

\begin{tabular}{|c|c|c|c|c|c|c|c|}
\hline \multirow[t]{2}{*}{ Compounds } & \multicolumn{2}{|c|}{$\begin{array}{l}\text { MMP13 Activity } \\
\text { (Fluorescence count) }\end{array}$} & \multicolumn{2}{|c|}{$\begin{array}{c}\text { Background } \\
\text { (Fluorescence count) }\end{array}$} & \multicolumn{2}{|c|}{$\%$ Activity } & \multirow[t]{2}{*}{$\%$ Inhibition } \\
\hline & Repeat1 & Repeat2 & Repeat1 & Repeat2 & Repeat1 & Repeat2 & \\
\hline No Compound & 9961 & 10322 & 705 & 985 & 100 & 100 & 0 \\
\hline NCGC00167526, $10 \mu \mathrm{M}$ & 7717 & 7857 & 653 & 773 & 76 & 76 & 24 \\
\hline NCGC00160398, $10 \mu \mathrm{M}$ & 3327 & 3360 & 999 & 1095 & 25 & 24 & 75 \\
\hline NCGC00025297, $10 \mu \mathrm{M}$ & 8927 & 8870 & 786 & 792 & 88 & 87 & 13 \\
\hline Batimastat, $0.01 \mu \mathrm{M}$ & 9094 & 9008 & 846 & 793 & 89 & 88 & 11 \\
\hline Batimastat, $0.1 \mu \mathrm{M}$ & 6273 & 6230 & 823 & 809 & 59 & 58 & 42 \\
\hline Batimastat, $1 \mu \mathrm{M}$ & 1221 & 1221 & 705 & 713 & 5 & 5 & 95 \\
\hline Background & 155 & 157 & 150 & 149 & & & \\
\hline
\end{tabular}

MMP13 Activity

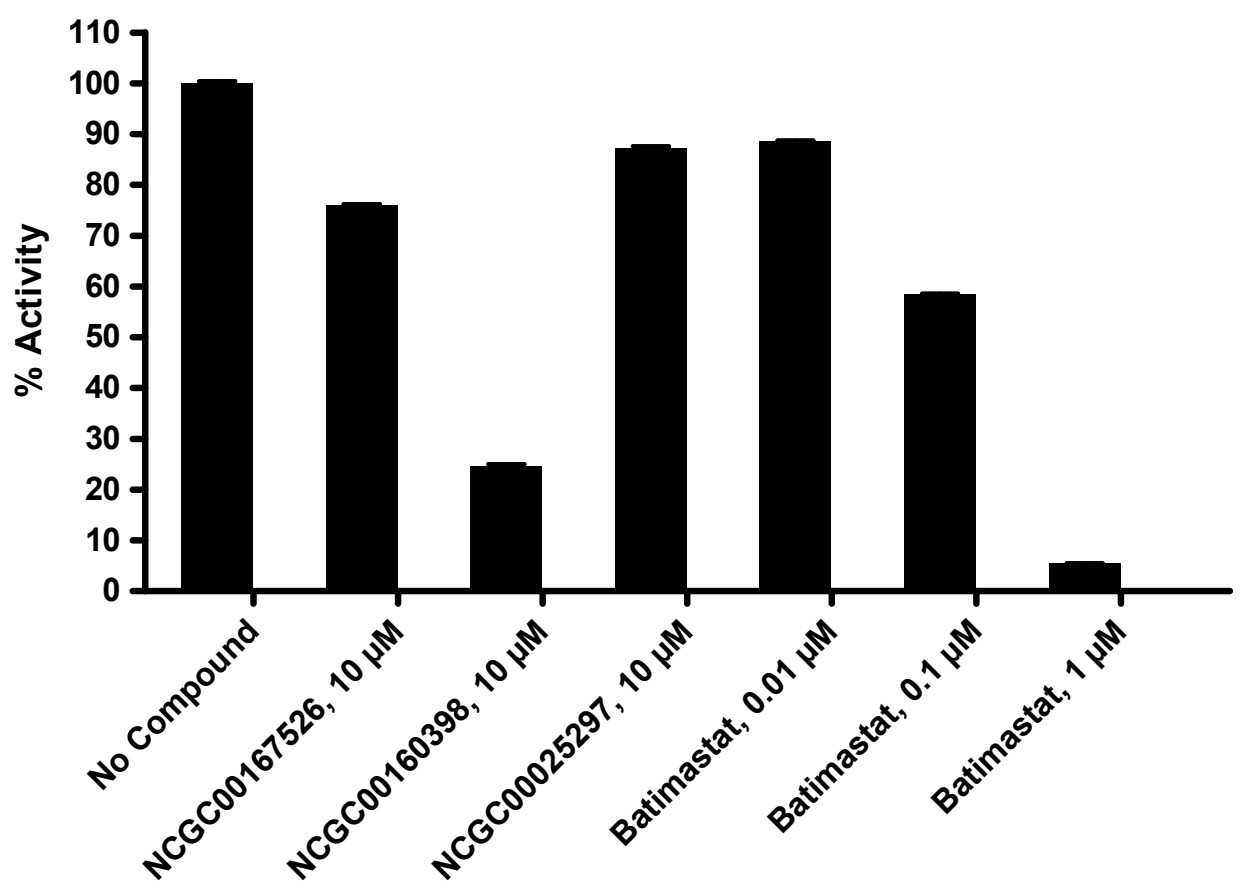


6042 Cornerstone Court West, Suite B

San Diego, CA 92121

Tel: 1.858 .829 .3082

Fax: 1.858 .481 .8694

Email: info@bpsbioscience.com

\subsubsection{DPP3}

Table 3.2.35. Data for the Effect of the Compounds on DPP3 Activity

\begin{tabular}{|c|c|c|c|c|c|c|c|}
\hline \multirow[t]{2}{*}{ Compounds } & \multicolumn{2}{|c|}{$\begin{array}{c}\text { DPP3 Activity } \\
\text { (Fluorescence count) }\end{array}$} & \multicolumn{2}{|c|}{$\begin{array}{c}\text { Background } \\
\text { (Fluorescence count) }\end{array}$} & \multicolumn{2}{|c|}{$\%$ Activity } & \multirow[t]{2}{*}{$\%$ Inhibition } \\
\hline & Repeat1 & Repeat2 & Repeat1 & Repeat2 & Repeat1 & Repeat2 & \\
\hline No Compound & 44056 & 46143 & 3900 & 3999 & 98 & 102 & 0 \\
\hline NCGC00167526, $10 \mu \mathrm{M}$ & 42024 & 41828 & 4409 & 3715 & 91 & 93 & 8 \\
\hline NCGC00160398, $10 \mu \mathrm{M}$ & 43746 & 44531 & 3950 & 4271 & 97 & 98 & 3 \\
\hline NCGC00025297, $10 \mu \mathrm{M}$ & 44179 & 43330 & 4097 & 4267 & 97 & 95 & 4 \\
\hline Spinorphine, $0.1 \mu \mathrm{M}$ & 45714 & 50360 & 3738 & 3658 & 102 & 114 & 0 \\
\hline Spinorphine, $1 \mu \mathrm{M}$ & 9183 & 8460 & 1211 & 1335 & 19 & 17 & 82 \\
\hline Spinorphine, $10 \mu \mathrm{M}$ & 1716 & 1819 & 1139 & 1116 & 1 & 1 & 99 \\
\hline Background & 1313 & 1356 & 1058 & 1078 & & & \\
\hline
\end{tabular}

DPP3 activity

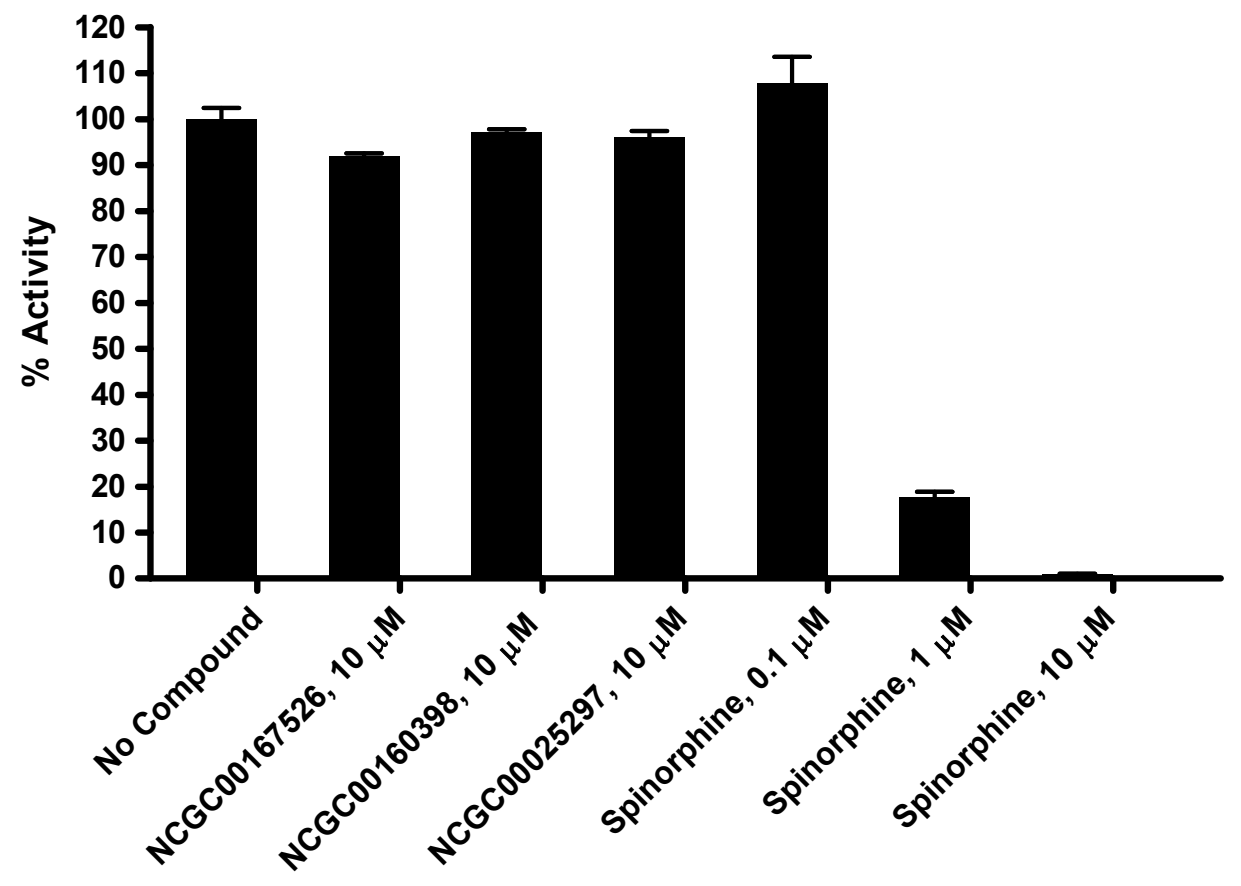


6042 Cornerstone Court West, Suite B

San Diego, CA 92121

Tel: 1.858 .829 .3082

Fax: 1.858 .481 .8694

Email: info@bpsbioscience.com

\subsubsection{DPP4}

Table 3.2.36. Data for the Effect of the Compounds on DPP4 Activity

\begin{tabular}{|c|c|c|c|c|c|c|c|}
\hline \multirow[t]{2}{*}{ Compounds } & \multicolumn{2}{|c|}{$\begin{array}{l}\text { DPP4 Activity } \\
\text { (Fluorescence count) }\end{array}$} & \multicolumn{2}{|c|}{$\begin{array}{c}\text { Background } \\
\text { (Fluorescence count) }\end{array}$} & \multicolumn{2}{|c|}{$\%$ Activity } & \multirow[t]{2}{*}{$\%$ Inhibition } \\
\hline & Repeat1 & Repeat2 & Repeat1 & Repeat2 & Repeat1 & Repeat2 & \\
\hline No Compound & 18664 & 18230 & 3486 & 3255 & 101 & 99 & 0 \\
\hline NCGC00167526, $10 \mu \mathrm{M}$ & 14545 & 15960 & 2756 & 2799 & 78 & 87 & 17 \\
\hline NCGC00160398, $10 \mu \mathrm{M}$ & 15176 & 15667 & 2921 & 3110 & 81 & 83 & 18 \\
\hline NCGC00025297, $10 \mu \mathrm{M}$ & 14687 & 14879 & 2760 & 2848 & 79 & 80 & 21 \\
\hline Sitagliptin, $0.001 \mu \mathrm{M}$ & 14862 & 15705 & 2196 & 2265 & 84 & 89 & 13 \\
\hline Sitagliptin, $0.01 \mu \mathrm{M}$ & 8929 & 8882 & 1830 & 1723 & 47 & 47 & 53 \\
\hline Sitagliptin, $0.1 \mu \mathrm{M}$ & 2437 & 2454 & 1318 & 1365 & 7 & 7 & 93 \\
\hline Background & 1276 & 1839 & 1195 & 1755 & & & \\
\hline
\end{tabular}

DPP4 activity

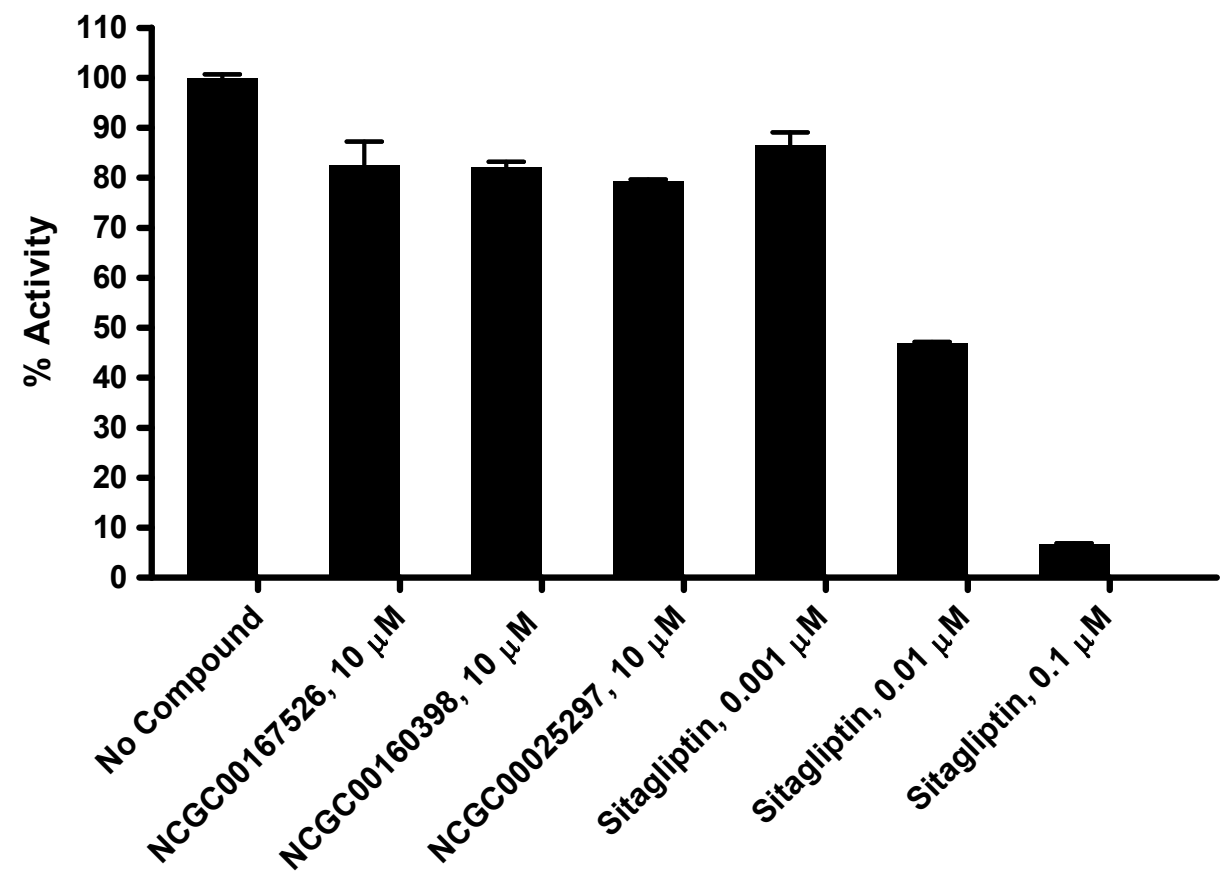


6042 Cornerstone Court West, Suite B

San Diego, CA 92121

Tel: 1.858 .829 .3082

Fax: 1.858 .481 .8694

Email: info@bpsbioscience.com

\subsubsection{DPP7}

Table 3.2.37. Data for the Effect of the Compounds on DPP7 Activity

\begin{tabular}{|c|c|c|c|c|c|c|c|}
\hline \multirow[t]{2}{*}{ Compounds } & \multicolumn{2}{|c|}{$\begin{array}{c}\text { DPP7 Activity } \\
\text { (Fluorescence count) }\end{array}$} & \multicolumn{2}{|c|}{$\begin{array}{c}\text { Background } \\
\text { (Fluorescence count) }\end{array}$} & \multicolumn{2}{|c|}{$\%$ Activity } & \multirow[t]{2}{*}{$\%$ Inhibitior } \\
\hline & Repeat1 & Repeat2 & Repeat1 & Repeat2 & Repeat1 & Repeat2 & \\
\hline No Compound & 4790 & 4678 & 566 & 485 & 100 & 100 & 0 \\
\hline NCGC00167526, $10 \mu \mathrm{M}$ & 3729 & 3792 & 380 & 388 & 80 & 81 & 20 \\
\hline NCGC00160398, $10 \mu \mathrm{M}$ & 3241 & 3507 & 375 & 403 & 68 & 74 & 29 \\
\hline NCGC00025297, $10 \mu \mathrm{M}$ & 660 & 1307 & 259 & 292 & 9 & 24 & 83 \\
\hline $\mathrm{KR} 62436,1 \mu \mathrm{M}$ & 4110 & 4599 & 472 & 469 & 86 & 98 & 8 \\
\hline $\mathrm{KR} 62436,10 \mu \mathrm{M}$ & 3018 & 3620 & 323 & 393 & 64 & 77 & 30 \\
\hline $\mathrm{KR} 62436,100 \mu \mathrm{M}$ & 1206 & 1304 & 229 & 295 & 23 & 24 & 77 \\
\hline Background & 209 & 252 & 195 & 242 & & & \\
\hline
\end{tabular}

DPP7 Activity

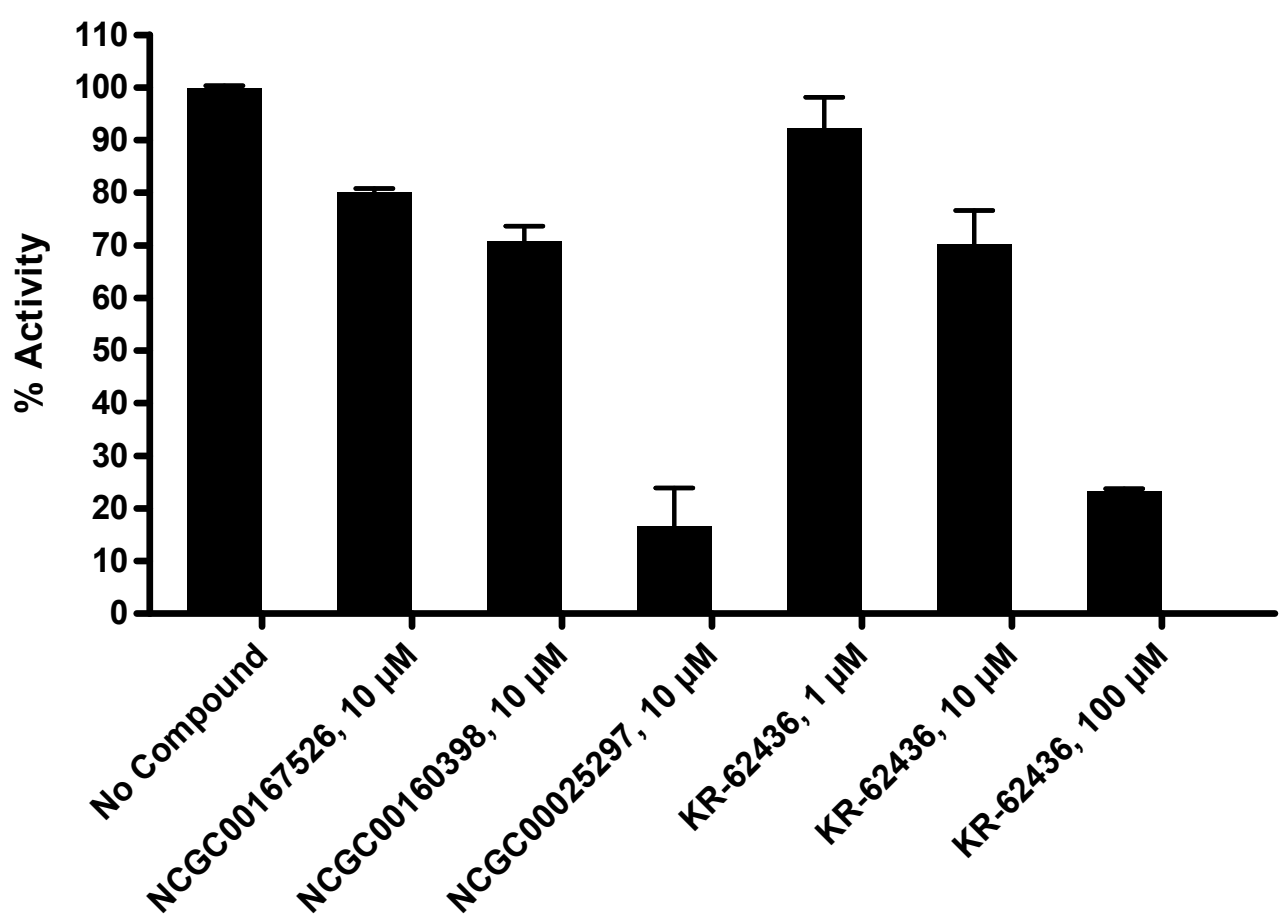


6042 Cornerstone Court West, Suite B

San Diego, CA 92121

Tel: 1.858 .829 .3082

Fax: 1.858 .481 .8694

Email: info@bpsbioscience.com

\subsubsection{DPP8}

Table 3.2.38. Data for the Effect of the Compounds on DPP8 Activity

\begin{tabular}{|c|c|c|c|c|c|c|c|}
\hline \multirow[t]{2}{*}{ Compounds } & \multicolumn{2}{|c|}{$\begin{array}{l}\text { DPP8 Activity } \\
\text { (Fluorescence count) }\end{array}$} & \multicolumn{2}{|c|}{$\begin{array}{c}\text { Background } \\
\text { (Fluorescence count) }\end{array}$} & \multicolumn{2}{|c|}{$\%$ Activity } & \multirow[t]{2}{*}{$\%$ Inhibition } \\
\hline & Repeat1 & Repeat2 & Repeat1 & Repeat2 & Repeat1 & Repeat2 & \\
\hline No Compound & 12015 & 10890 & 1835 & 1616 & 105 & 95 & 0 \\
\hline NCGC00167526, $10 \mu \mathrm{M}$ & 10224 & 10376 & 1094 & 1602 & 94 & 90 & 8 \\
\hline NCGC00160398, $10 \mu \mathrm{M}$ & 10514 & 10167 & 1364 & 1390 & 94 & 90 & 8 \\
\hline NCGC00025297, $10 \mu \mathrm{M}$ & 9190 & 10396 & 1049 & 1665 & 84 & 90 & 13 \\
\hline $\mathrm{KR} 62436,1 \mu \mathrm{M}$ & 11007 & 11277 & 1348 & 1374 & 99 & 102 & 0 \\
\hline $\mathrm{KR} 62436,10 \mu \mathrm{M}$ & 10133 & 8763 & 1530 & 804 & 88 & 82 & 15 \\
\hline $\mathrm{KR} 62436,100 \mu \mathrm{M}$ & 2112 & 2332 & 310 & 336 & 18 & 20 & 81 \\
\hline Background & 247 & 216 & 230 & 210 & & & \\
\hline
\end{tabular}

DPP8 Activity

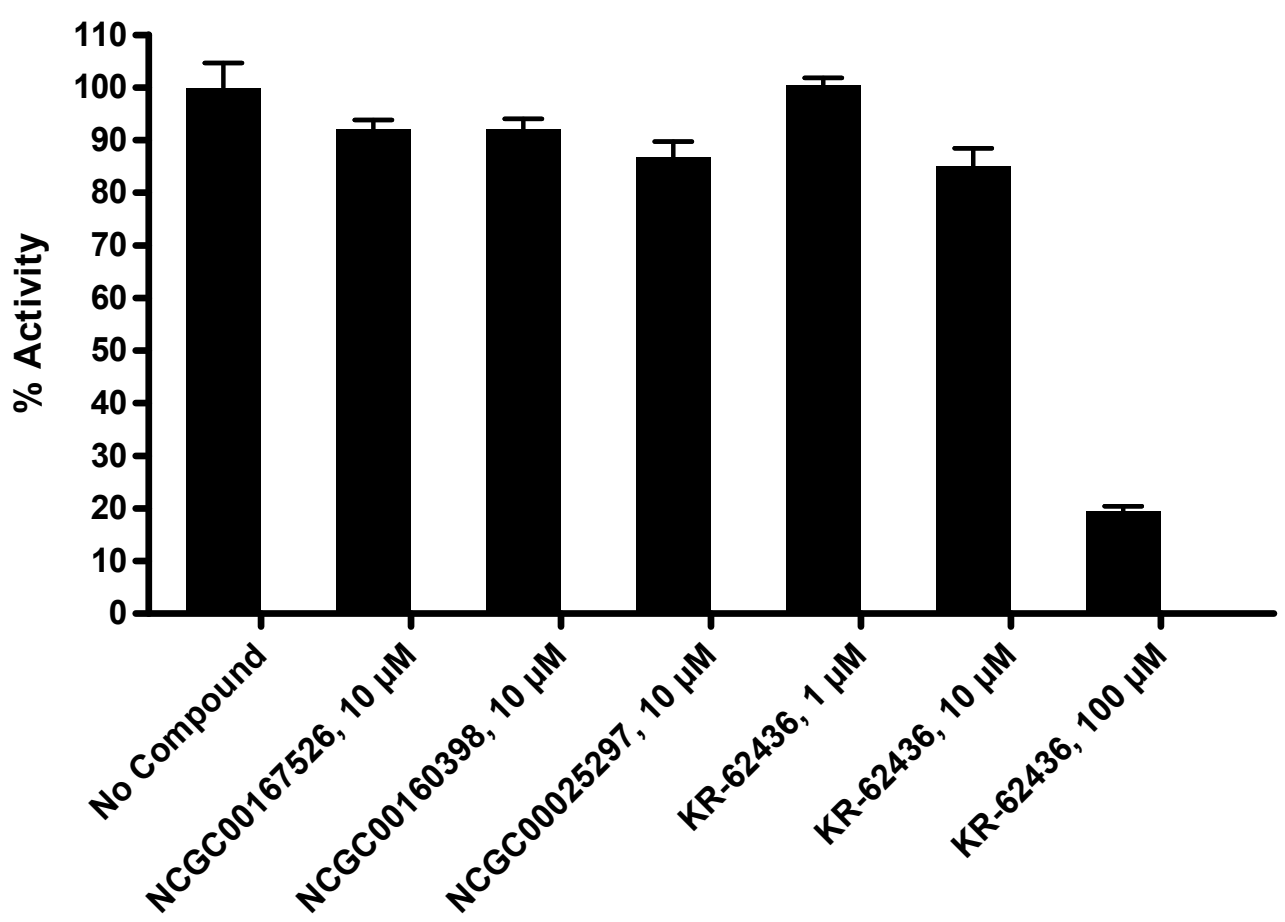


6042 Cornerstone Court West, Suite B

San Diego, CA 92121

Tel: 1.858 .829 .3082

Fax: 1.858 .481 .8694

Email: info@bpsbioscience.com

\subsubsection{DPP9}

Table 3.2.39. Data for the Effect of the Compounds on DPP9 Activity

\begin{tabular}{|c|c|c|c|c|c|c|c|}
\hline \multirow{2}{*}{ Compounds } & \multicolumn{2}{|c|}{$\begin{array}{l}\text { DPP9 Activity } \\
\text { (Fluorescence count) }\end{array}$} & \multicolumn{2}{|c|}{$\begin{array}{c}\text { Background } \\
\text { (Fluorescence count) }\end{array}$} & \multicolumn{2}{|c|}{$\%$ Activity } & \multirow[t]{2}{*}{$\%$ Inhibition } \\
\hline & Repeat1 & Repeat2 & Repeat1 & Repeat2 & Repeat1 & Repeat2 & \\
\hline No Compound & 6728 & 6726 & 1082 & 1102 & 100 & 100 & 0 \\
\hline NCGC00167526, $10 \mu \mathrm{M}$ & 5462 & 5396 & 829 & 819 & 82 & 81 & 18 \\
\hline NCGC00160398, $10 \mu \mathrm{M}$ & 5499 & 6006 & 808 & 923 & 83 & 90 & 13 \\
\hline NCGC00025297, $10 \mu \mathrm{M}$ & 5644 & 5849 & 838 & 914 & 85 & 88 & 14 \\
\hline KR62436, $0.1 \mu \mathrm{M}$ & 6633 & 7122 & 999 & 1125 & 100 & 106 & 0 \\
\hline $\mathrm{KR} 62436,1 \mu \mathrm{M}$ & 3343 & 3659 & 373 & 877 & 53 & 49 & 49 \\
\hline $\mathrm{KR} 62436,10 \mu \mathrm{M}$ & 1007 & 1018 & 269 & 249 & 13 & 14 & 87 \\
\hline Background & 220 & 225 & 230 & 228 & & & \\
\hline
\end{tabular}

DPP9 Activity

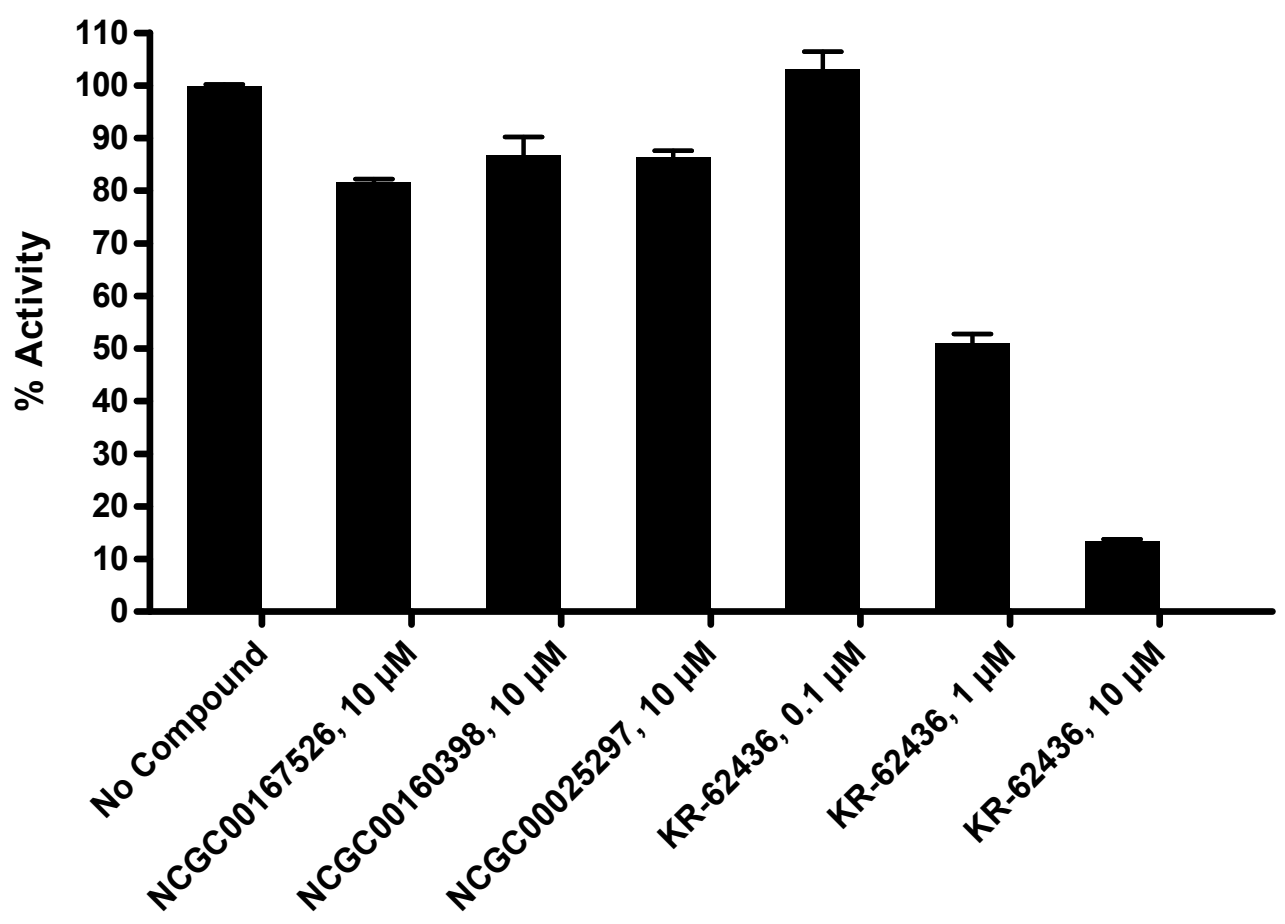




\section{Cornerstone Court West, Suite B}

San Diego, CA 92121

Tel: 1.858 .829 .3082

Fax: 1.858 .481 .8694

Email: info@bpsbioscience.com

\subsubsection{FAP}

Table 3.2.40. Data for the Effect of the Compounds on FAP Activity

\begin{tabular}{|c|c|c|c|c|c|c|c|}
\hline \multirow[t]{2}{*}{ Compounds } & \multicolumn{2}{|c|}{$\begin{array}{c}\text { FAP Activity } \\
\text { (Fluorescence count) }\end{array}$} & \multicolumn{2}{|c|}{$\begin{array}{c}\text { Background } \\
\text { (Fluorescence count) }\end{array}$} & \multicolumn{2}{|c|}{$\%$ Activity } & \multirow[t]{2}{*}{$\%$ Inhibitior } \\
\hline & Repeat1 & Repeat2 & Repeat1 & Repeat2 & Repeat1 & Repeat2 & \\
\hline No Compound & 31645 & 31041 & 4753 & 4762 & 101 & 99 & 0 \\
\hline NCGC00167526, $10 \mu \mathrm{M}$ & 21101 & 21989 & 2816 & 3061 & 69 & 71 & 30 \\
\hline NCGC00160398, $10 \mu \mathrm{M}$ & 23833 & 23249 & 3786 & 3620 & 75 & 74 & 25 \\
\hline NCGC00025297, $10 \mu \mathrm{M}$ & 22699 & 22836 & 3896 & 3649 & 71 & 72 & 29 \\
\hline SP-13786, $0.001 \mu \mathrm{M}$ & 29765 & 30336 & 3599 & 3675 & 98 & 100 & 1 \\
\hline $\mathrm{SP}-13786,0.01 \mu \mathrm{M}$ & 20566 & 20174 & 3256 & 2970 & 65 & 65 & 35 \\
\hline SP-13786, 0.1 $\mu \mathrm{M}$ & 1365 & 1323 & 1258 & 1190 & 0 & 0 & 100 \\
\hline Background & 1242 & 1400 & 1191 & 1369 & & & \\
\hline
\end{tabular}

FAP Activity

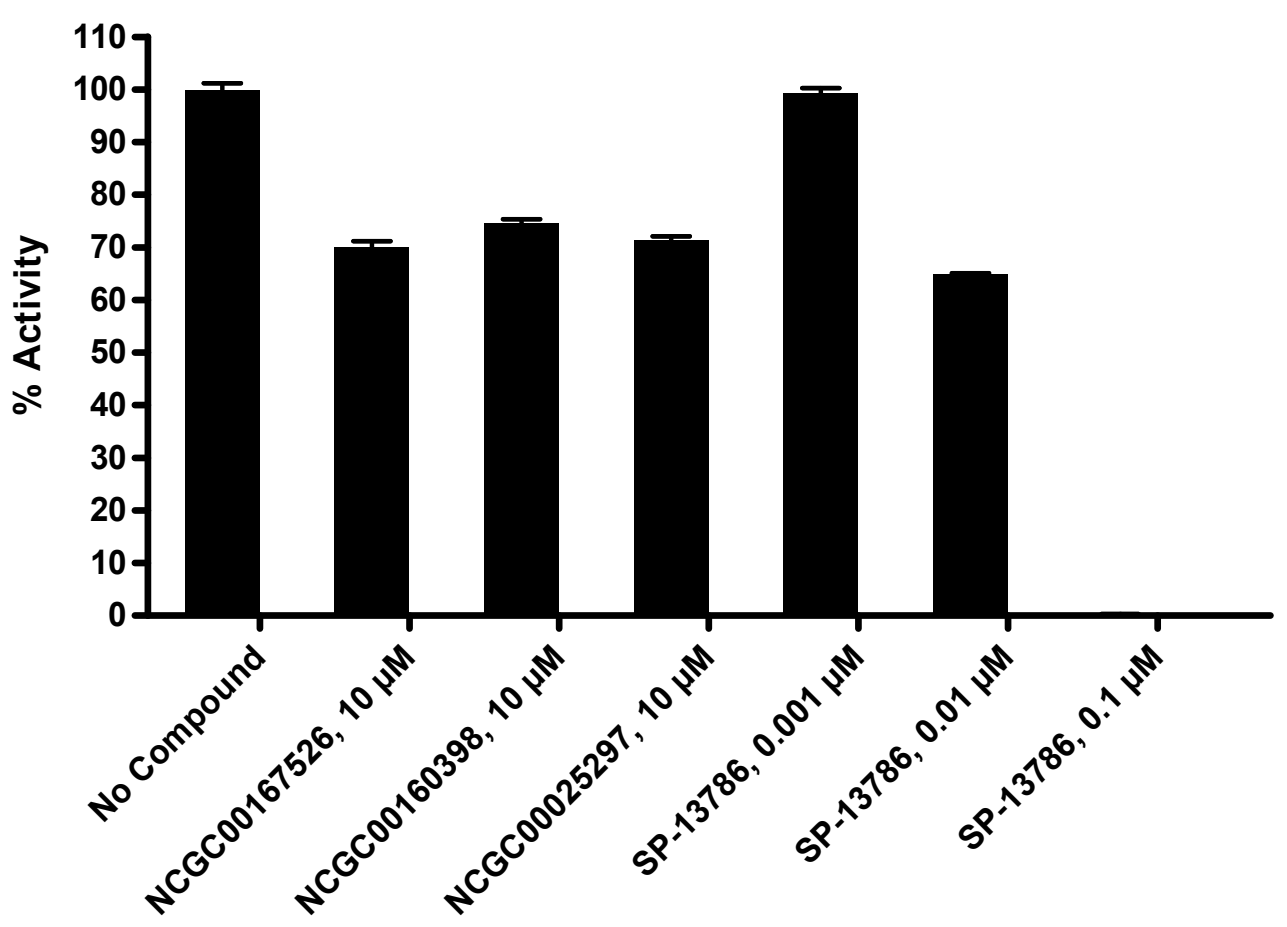


6042 Cornerstone Court West, Suite B

San Diego, CA 92121

Tel: 1.858 .829 .3082

Fax: 1.858 .481 .8694

Email: info@bpsbioscience.com

3.2.41. HCV1a (D168V)

Table 3.2.41. Data for the Effect of the Compounds on HCV1a (D168V) Activity

\begin{tabular}{|c|c|c|c|c|c|c|c|}
\hline \multirow[t]{2}{*}{ Compounds } & \multicolumn{2}{|c|}{$\begin{array}{c}\text { HCV1a (D168V) } \\
\text { Activity } \\
\text { (Fluorescence count) }\end{array}$} & \multicolumn{2}{|c|}{$\begin{array}{c}\text { Background } \\
\text { (Fluorescence count) }\end{array}$} & \multicolumn{2}{|c|}{$\%$ Activity } & \multirow[t]{2}{*}{$\%$ Inhibition } \\
\hline & Repeat1 & Repeat2 & Repeat1 & Repeat2 & Repeat1 & Repeat2 & \\
\hline No Compound & 7562 & 7826 & 1245 & 1252 & 98 & 102 & 0 \\
\hline NCGC00167526, $10 \mu \mathrm{M}$ & 7711 & 7444 & 1432 & 1431 & 97 & 93 & 5 \\
\hline NCGC00160398, $10 \mu \mathrm{M}$ & 36331 & 36122 & 33942 & 34067 & 36 & 33 & 66 \\
\hline NCGC00025297, $10 \mu \mathrm{M}$ & 7889 & 7604 & 1353 & 1424 & 101 & 96 & 1 \\
\hline Danoprevir, $0.001 \mu \mathrm{M}$ & 6378 & 6687 & 1267 & 1274 & 79 & 84 & 18 \\
\hline Danoprevir, $0.01 \mu \mathrm{M}$ & 4164 & 3648 & 1306 & 1231 & 45 & 37 & 59 \\
\hline Danoprevir, $0.1 \mu \mathrm{M}$ & 1804 & 1819 & 1254 & 1275 & 8 & 8 & 92 \\
\hline Background & 1245 & 1289 & 1243 & 1245 & & & \\
\hline
\end{tabular}

HCV1a (D168V) Activity

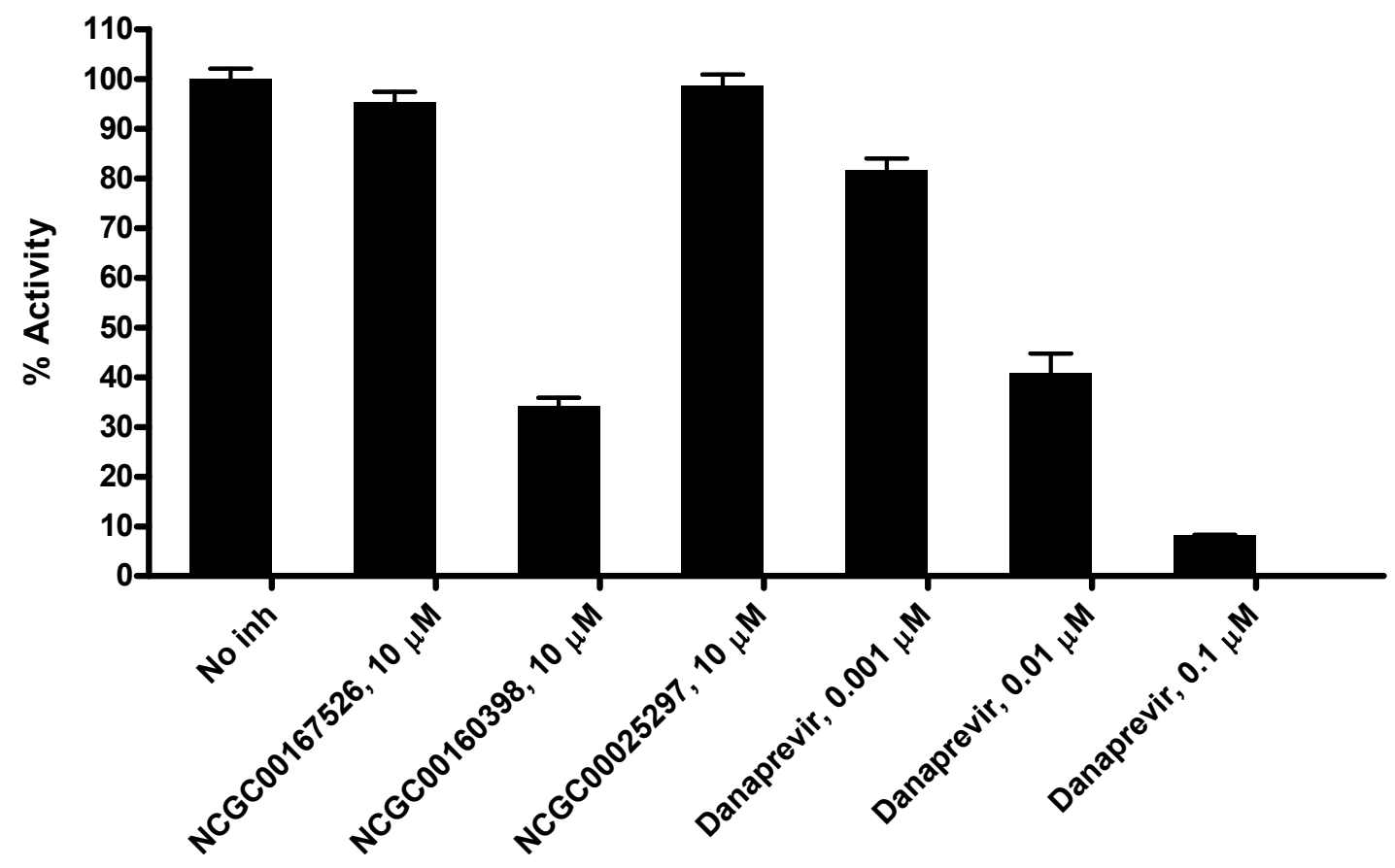


6042 Cornerstone Court West, Suite B

San Diego, CA 92121

Tel: 1.858 .829 .3082

Fax: 1.858 .481 .8694

Email: info@bpsbioscience.com

\subsubsection{HCV1b}

Table 3.2.42. Data for the Effect of the Compounds on HCV1b Activity

\begin{tabular}{|c|c|c|c|c|c|c|c|}
\hline \multirow{2}{*}{ Compounds } & \multicolumn{2}{|c|}{$\begin{array}{l}\text { HCV1b Activity } \\
\text { (Fluorescence count) }\end{array}$} & \multicolumn{2}{|c|}{$\begin{array}{c}\text { Background } \\
\text { (Fluorescence count) }\end{array}$} & \multicolumn{2}{|c|}{$\%$ Activity } & \multirow{2}{*}{$\%$ Inhibition } \\
\hline & Repeat1 & Repeat2 & Repeat1 & Repeat2 & Repeat1 & Repeat2 & \\
\hline No Compound & 6588 & 6456 & 1245 & 1252 & 101 & 99 & 0 \\
\hline NCGC00167526, $10 \mu \mathrm{M}$ & 6301 & 5968 & 1432 & 1431 & 92 & 86 & 11 \\
\hline NCGC00160398, $10 \mu \mathrm{M}$ & 36598 & 37154 & 33942 & 34067 & 49 & 60 & 46 \\
\hline NCGC00025297, $10 \mu \mathrm{M}$ & 5076 & 5148 & 1353 & 1424 & 70 & 71 & 29 \\
\hline Danoprevir, $0.0001 \mu \mathrm{M}$ & 4972 & 5357 & 1254 & 1263 & 70 & 78 & 26 \\
\hline Danoprevir, $0.001 \mu \mathrm{M}$ & 3474 & 3493 & 1267 & 1274 & 42 & 42 & 58 \\
\hline Danoprevir, $0.01 \mu \mathrm{M}$ & 1681 & 1573 & 1306 & 1231 & 8 & 6 & 93 \\
\hline Background & 1264 & 1252 & 1243 & 1245 & & & \\
\hline
\end{tabular}

HCV1b Activity

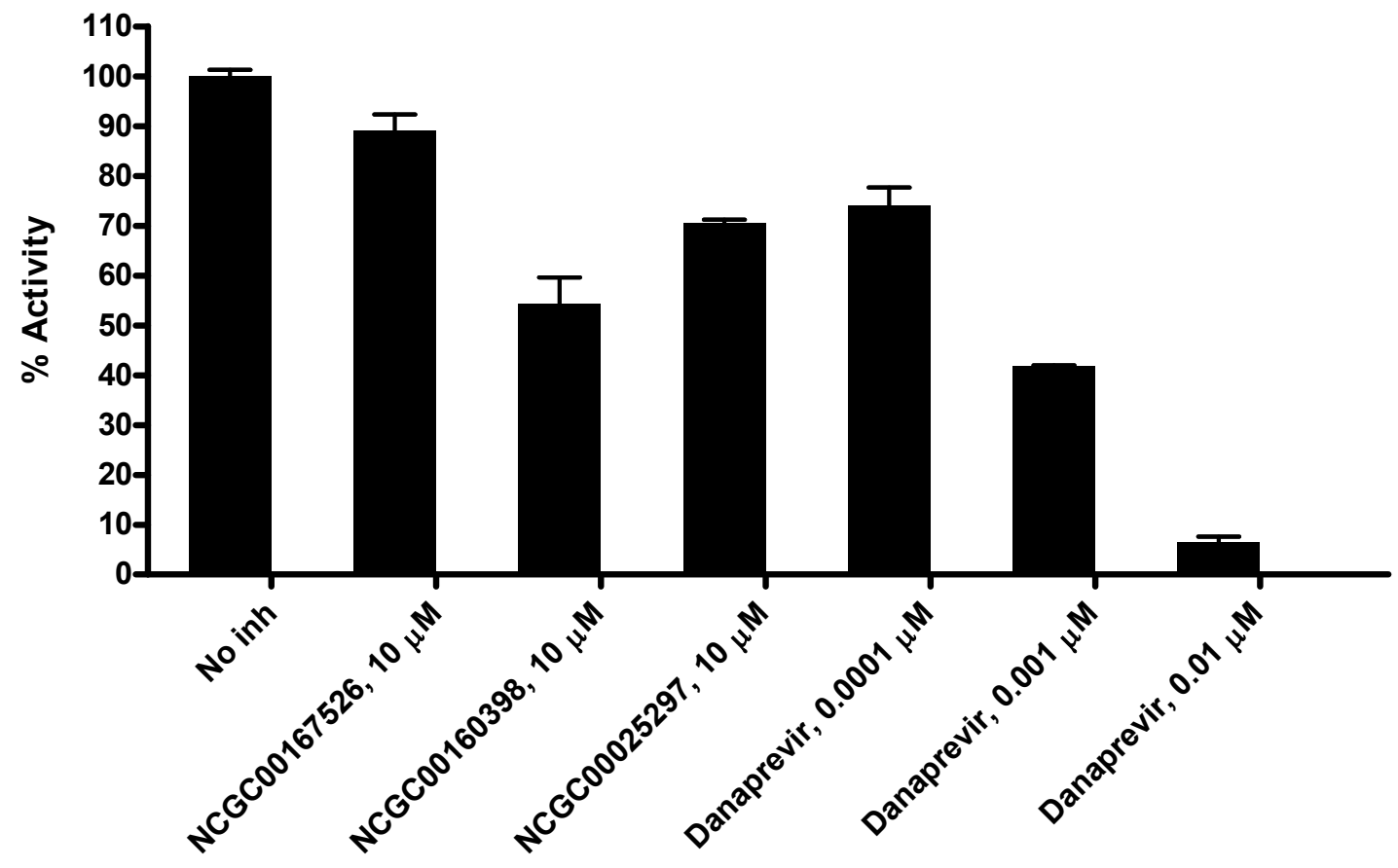


6042 Cornerstone Court West, Suite B

San Diego, CA 92121

Tel: 1.858 .829 .3082

Fax: 1.858 .481 .8694

Email: info@bpsbioscience.com

\subsubsection{HCV1b (D168V)}

Table 3.2.43. Data for the Effect of the Compounds on HCV1b (D168V) Activity

\begin{tabular}{|c|c|c|c|c|c|c|c|}
\hline \multirow[t]{2}{*}{ Compounds } & \multicolumn{2}{|c|}{$\begin{array}{c}\text { HCV1b (D168V) } \\
\text { Activity } \\
\text { (Fluorescence count) }\end{array}$} & \multicolumn{2}{|c|}{$\begin{array}{c}\text { Background } \\
\text { (Fluorescence count) }\end{array}$} & \multicolumn{2}{|c|}{$\%$ Activity } & \multirow[t]{2}{*}{$\%$ Inhibition } \\
\hline & Repeat1 & Repeat2 & Repeat1 & Repeat2 & Repeat1 & Repeat2 & \\
\hline No Compound & 10955 & 10093 & 1245 & 1252 & 105 & 95 & 0 \\
\hline NCGC00167526, $10 \mu \mathrm{M}$ & 10444 & 10085 & 1432 & 1431 & 97 & 93 & 5 \\
\hline NCGC00160398, $10 \mu \mathrm{M}$ & 39196 & 39178 & 33942 & 34067 & 56 & 56 & 44 \\
\hline NCGC00025297, $10 \mu \mathrm{M}$ & 9567 & 9389 & 1353 & 1424 & 88 & 86 & 13 \\
\hline Danoprevir, $0.001 \mu \mathrm{M}$ & 7798 & 7735 & 1267 & 1274 & 70 & 70 & 30 \\
\hline Danoprevir, $0.01 \mu \mathrm{M}$ & 3387 & 3293 & 1306 & 1231 & 23 & 22 & 78 \\
\hline Danoprevir, $0.1 \mu \mathrm{M}$ & 1457 & 1467 & 1254 & 1275 & 2 & 2 & 98 \\
\hline Background & 1257 & 1282 & 1243 & 1245 & & & \\
\hline
\end{tabular}

HCV1b (D168V) Activity

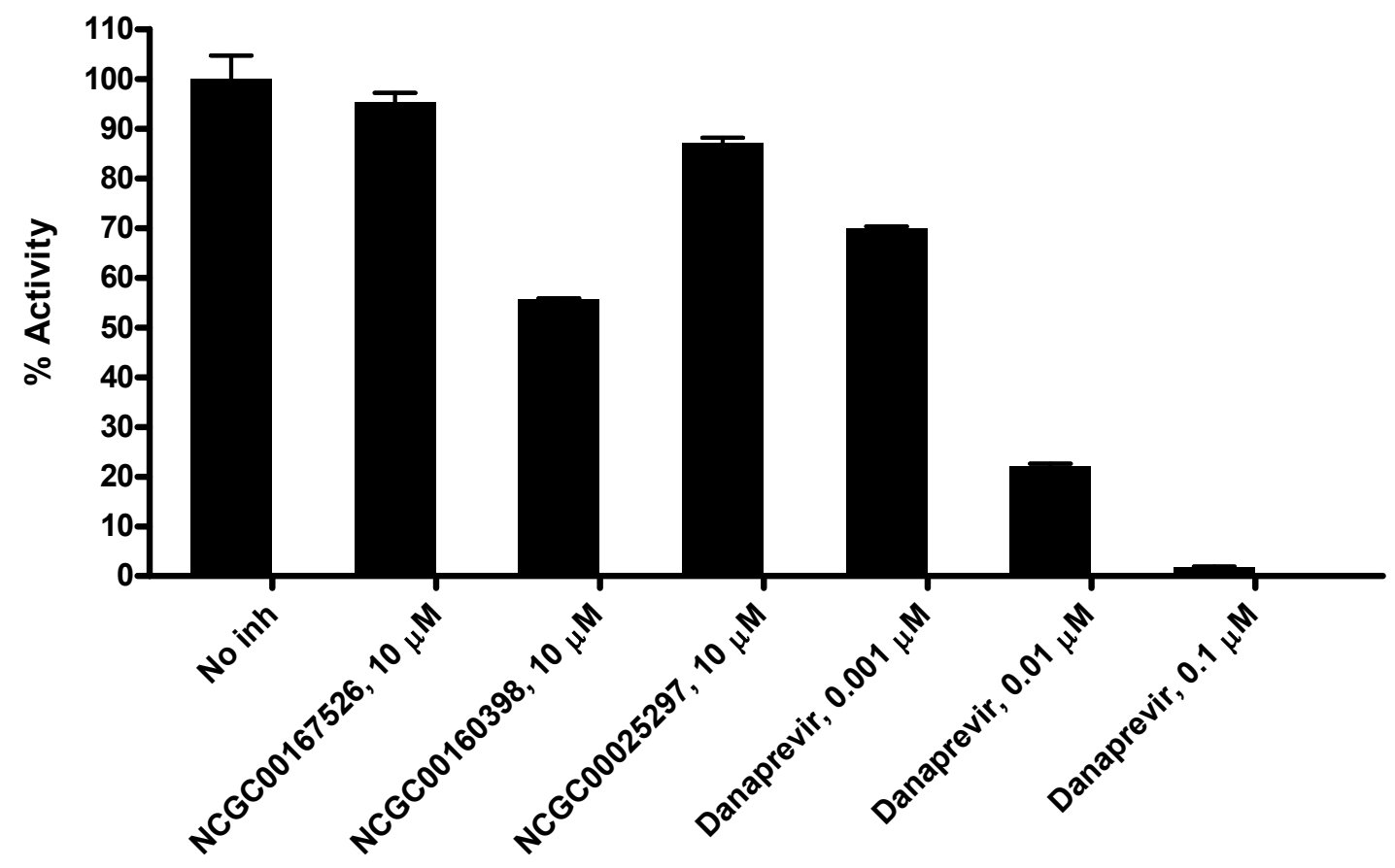


6042 Cornerstone Court West, Suite B
San Diego, CA 92121

Tel: 1.858 .829 .3082

Fax: 1.858 .481 .8694

Email: info@bpsbioscience.com

\subsubsection{HCV1b (R155K)}

Table 3.2.44. Data for the Effect of the Compounds on HCV1b (R155K) Activity

\begin{tabular}{|c|c|c|c|c|c|c|c|}
\hline \multirow[t]{2}{*}{ Compounds } & \multicolumn{2}{|c|}{$\begin{array}{c}\text { HCV1b (R155K) } \\
\text { Activity } \\
\text { (Fluorescence count) }\end{array}$} & \multicolumn{2}{|c|}{$\begin{array}{c}\text { Background } \\
\text { (Fluorescence count) }\end{array}$} & \multicolumn{2}{|c|}{$\%$ Activity } & \multirow[t]{2}{*}{$\%$ Inhibition } \\
\hline & Repeat1 & Repeat2 & Repeat1 & Repeat2 & Repeat1 & Repeat2 & \\
\hline No Compound & 14521 & 14486 & 1245 & 1252 & 100 & 100 & 0 \\
\hline NCGC00167526, $10 \mu \mathrm{M}$ & 14539 & 14462 & 1432 & 1431 & 99 & 98 & 1 \\
\hline NCGC00160398, $10 \mu \mathrm{M}$ & 42200 & 41543 & 33942 & 34067 & 62 & 57 & 41 \\
\hline NCGC00025297, $10 \mu \mathrm{M}$ & 14743 & 15055 & 1353 & 1424 & 101 & 103 & 0 \\
\hline Danoprevir, $0.01 \mu \mathrm{M}$ & 12136 & 12244 & 1306 & 1231 & 82 & 83 & 18 \\
\hline Danoprevir, $0.1 \mu \mathrm{M}$ & 7275 & 6806 & 1254 & 1275 & 45 & 42 & 57 \\
\hline Danoprevir, $1 \mu \mathrm{M}$ & 2129 & 2062 & 1319 & 1376 & 6 & 5 & 94 \\
\hline Background & 1248 & 1281 & 1243 & 1245 & & & \\
\hline
\end{tabular}

HCV1b (R155K) Activity

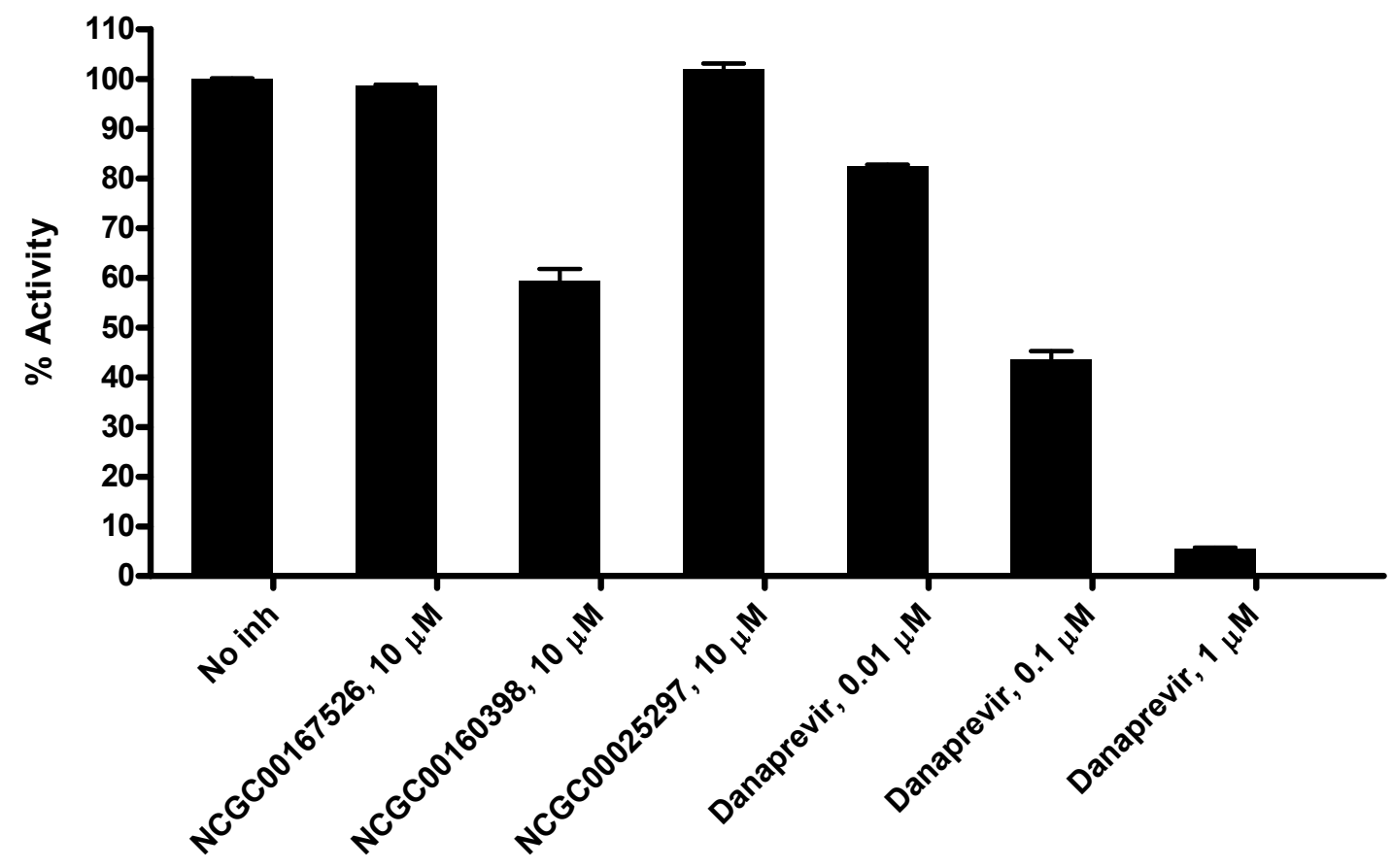


6042 Cornerstone Court West, Suite B
San Diego, CA 92121

Tel: 1.858 .829 .3082

Fax: 1.858 .481 .8694

Email: info@bpsbioscience.com

\subsubsection{HCV1b (R1550)}

Table 3.2.45. Data for the Effect of the Compounds on HCV1b (R155Q) Activity

\begin{tabular}{|c|c|c|c|c|c|c|c|}
\hline \multirow[t]{2}{*}{ Compounds } & \multicolumn{2}{|c|}{$\begin{array}{c}\text { HCV1b (R155Q) } \\
\text { Activity } \\
\text { (Fluorescence count) }\end{array}$} & \multicolumn{2}{|c|}{$\begin{array}{c}\text { Background } \\
\text { (Fluorescence count) }\end{array}$} & \multicolumn{2}{|c|}{$\%$ Activity } & \multirow[t]{2}{*}{$\%$ Inhibition } \\
\hline & Repeat1 & Repeat2 & Repeat1 & Repeat2 & Repeat1 & Repeat2 & \\
\hline No Compound & 8468 & 8345 & 1245 & 1252 & 101 & 99 & 0 \\
\hline NCGC00167526, $10 \mu \mathrm{M}$ & 8433 & 8010 & 1432 & 1431 & 98 & 92 & 5 \\
\hline NCGC00160398, $10 \mu \mathrm{M}$ & 36677 & 37354 & 33942 & 34067 & 37 & 47 & 58 \\
\hline NCGC00025297, $10 \mu \mathrm{M}$ & 8003 & 7388 & 1353 & 1424 & 92 & 84 & 12 \\
\hline Danoprevir, $0.01 \mu \mathrm{M}$ & 6754 & 6904 & 1306 & 1231 & 77 & 79 & 22 \\
\hline Danoprevir, $0.1 \mu \mathrm{M}$ & 4774 & 4836 & 1254 & 1275 & 49 & 50 & 51 \\
\hline Danoprevir, $1 \mu \mathrm{M}$ & 1377 & 1452 & 1319 & 1376 & 0 & 1 & 99 \\
\hline Background & 1264 & 1245 & 1243 & 1245 & & & \\
\hline
\end{tabular}

HCV1b (R155Q) Activity

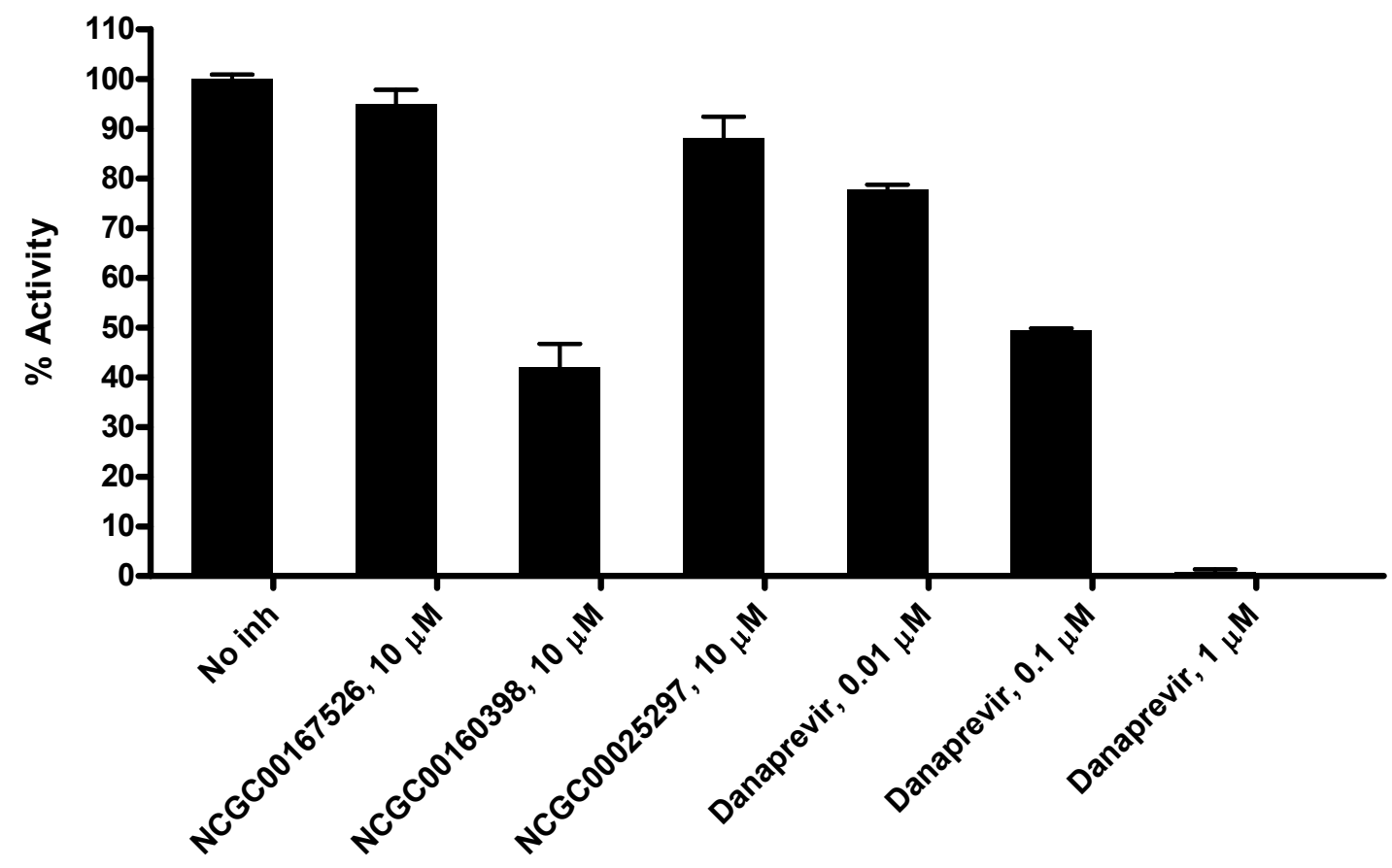


6042 Cornerstone Court West, Suite B

San Diego, CA 92121

Tel: 1.858 .829 .3082

Fax: 1.858 .481 .8694

Email: info@bpsbioscience.com

\subsubsection{HCV2a}

Table 3.2.46. Data for the Effect of the Compounds on HCV2a Activity

\begin{tabular}{|c|c|c|c|c|c|c|c|}
\hline \multirow{2}{*}{ Compounds } & \multicolumn{2}{|c|}{$\begin{array}{l}\text { HCV2a Activity } \\
\text { (Fluorescence count) }\end{array}$} & \multicolumn{2}{|c|}{$\begin{array}{c}\text { Background } \\
\text { (Fluorescence count) }\end{array}$} & \multicolumn{2}{|c|}{$\%$ Activity } & \multirow{2}{*}{$\%$ Inhibition } \\
\hline & Repeat1 & Repeat2 & Repeat1 & Repeat2 & Repeat1 & Repeat2 & \\
\hline No Compound & 21181 & 20912 & 1245 & 1252 & 101 & 99 & 0 \\
\hline NCGC00167526, $10 \mu \mathrm{M}$ & 20492 & 21471 & 1432 & 1431 & 96 & 101 & 1 \\
\hline NCGC00160398, $10 \mu \mathrm{M}$ & 49497 & 50236 & 33942 & 34067 & 78 & 82 & 20 \\
\hline NCGC00025297, $10 \mu \mathrm{M}$ & 21575 & 20727 & 1353 & 1424 & 102 & 98 & 0 \\
\hline Danoprevir, $0.001 \mu \mathrm{M}$ & 19070 & 18665 & 1267 & 1274 & 90 & 88 & 11 \\
\hline Danoprevir, $0.01 \mu \mathrm{M}$ & 10037 & 10142 & 1306 & 1231 & 44 & 45 & 55 \\
\hline Danoprevir, $0.1 \mu \mathrm{M}$ & 1937 & 1773 & 1254 & 1275 & 3 & 2 & 97 \\
\hline Background & 1259 & 1269 & 1243 & 1245 & & & \\
\hline
\end{tabular}

HCV2a Activity

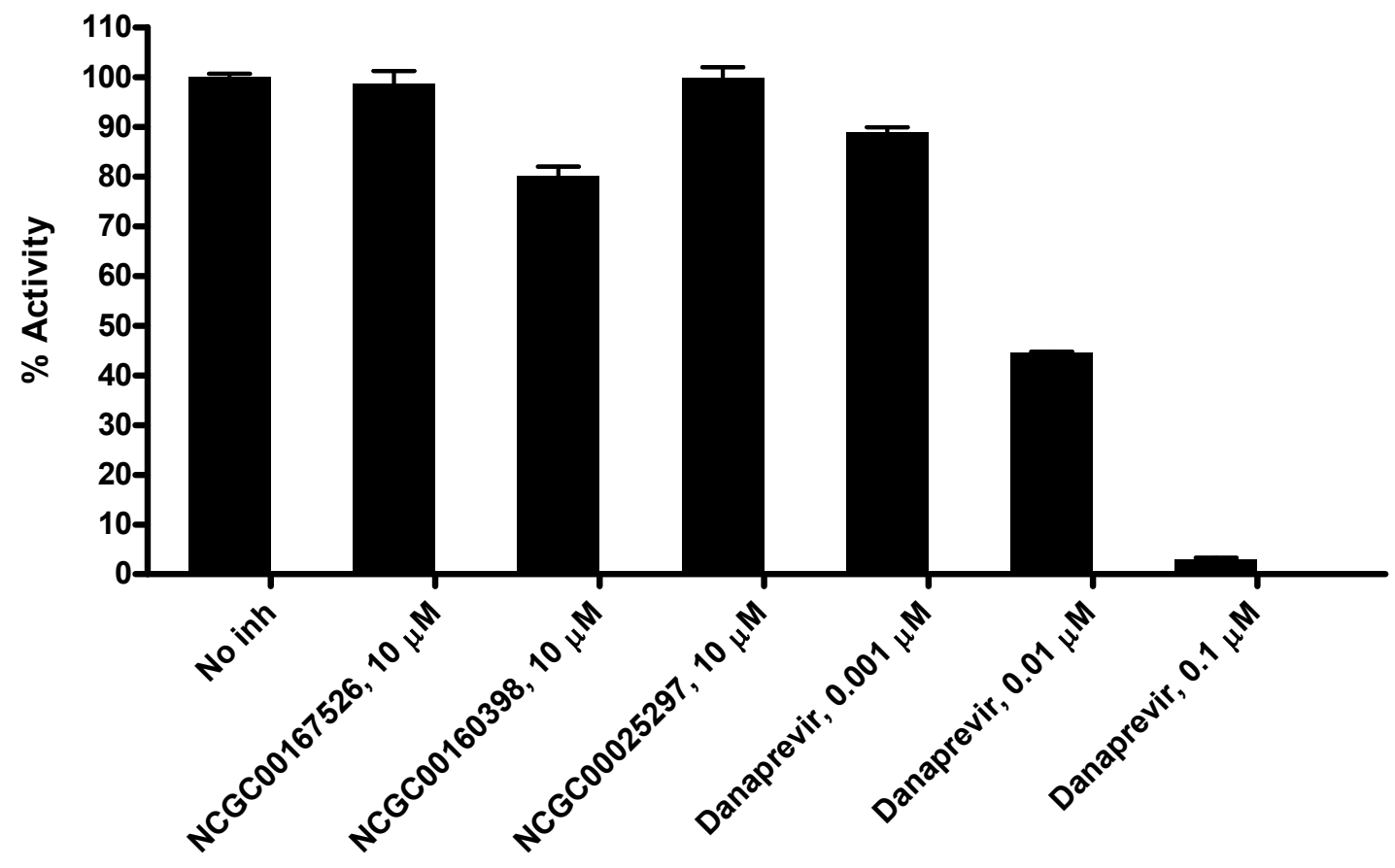


6042 Cornerstone Court West, Suite B

San Diego, CA 92121

Tel: 1.858 .829 .3082

Fax: 1.858 .481 .8694

Email: info@bpsbioscience.com

\subsubsection{POP}

Table 3.2.47. Data for the Effect of the Compounds on POP Activity

\begin{tabular}{|c|c|c|c|c|c|c|c|}
\hline \multirow{2}{*}{ Compounds } & \multicolumn{2}{|c|}{$\begin{array}{c}\text { POP Activity } \\
\text { (Fluorescence count) }\end{array}$} & \multicolumn{2}{|c|}{$\begin{array}{c}\text { Background } \\
\text { (Fluorescence count) }\end{array}$} & \multicolumn{2}{|c|}{$\%$ Activity } & \multirow[t]{2}{*}{$\%$ Inhibition } \\
\hline & Repeat1 & Repeat2 & Repeat1 & Repeat2 & Repeat1 & Repeat2 & \\
\hline No Compound & 11881 & 12573 & 2048 & 2028 & 96 & 104 & 0 \\
\hline NCGC00167526, $10 \mu \mathrm{M}$ & 8714 & 8865 & 1697 & 1891 & 69 & 68 & 31 \\
\hline NCGC00160398, $10 \mu \mathrm{M}$ & 9486 & 9244 & 2251 & 2022 & 71 & 71 & 29 \\
\hline NCGC00025297, $10 \mu \mathrm{M}$ & 9558 & 9160 & 1939 & 1899 & 75 & 71 & 27 \\
\hline Pro-Pro-CHO, $0.001 \mu \mathrm{M}$ & 10899 & 11358 & 1660 & 1665 & 91 & 95 & 7 \\
\hline Pro-Pro-CHO, $0.01 \mu \mathrm{M}$ & 8688 & 9166 & 1622 & 1685 & 69 & 73 & 29 \\
\hline Pro-Pro-CHO, $0.1 \mu \mathrm{M}$ & 1156 & 1159 & 1163 & 1175 & 0 & 0 & 100 \\
\hline Background & 1278 & 1233 & 1275 & 1190 & & & \\
\hline
\end{tabular}

POP Activity

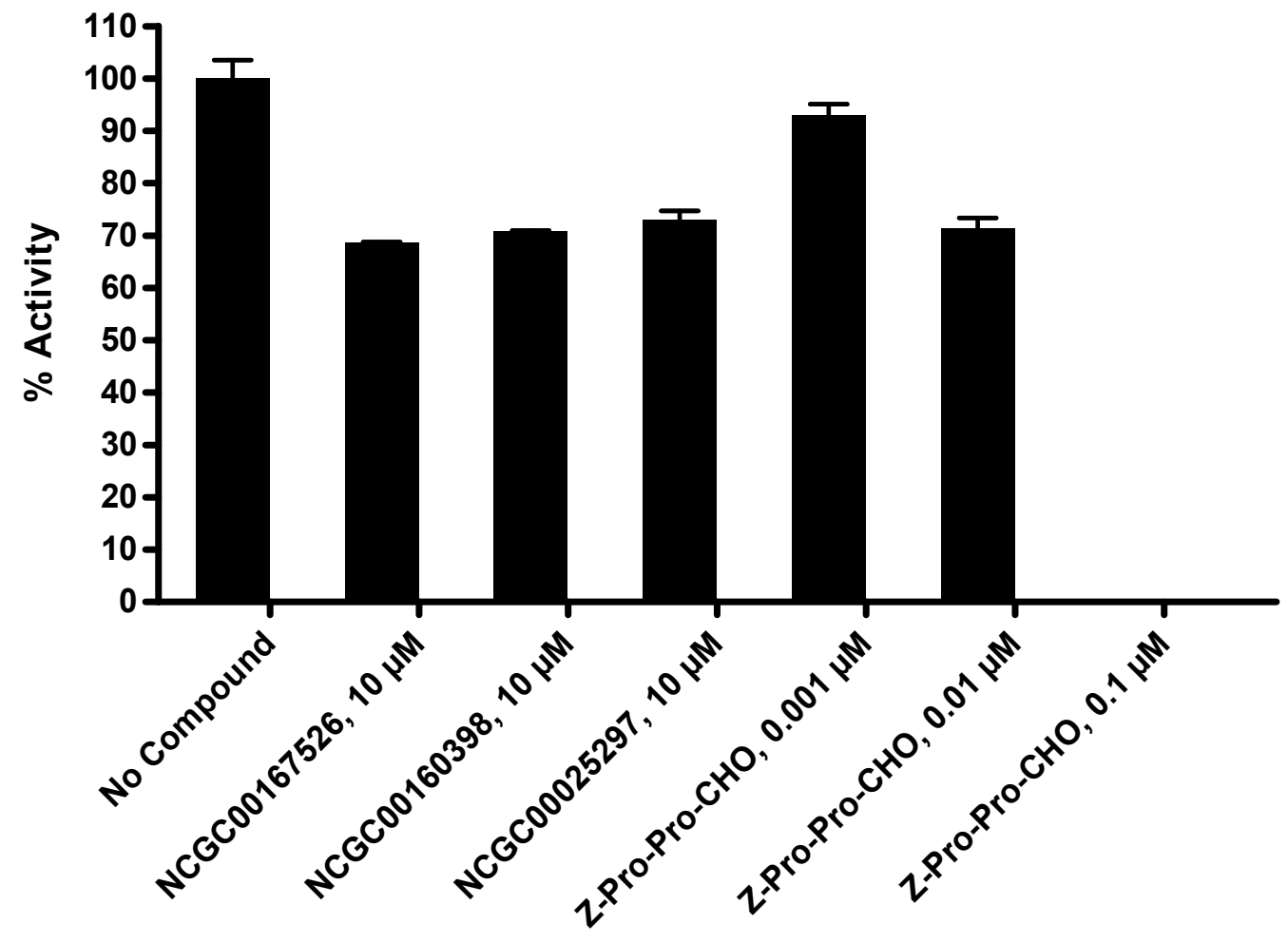


6042 Cornerstone Court West, Suite B

San Diego, CA 92121

Tel: 1.858 .829 .3082

Fax: 1.858 .481 .8694

Email: info@bpsbioscience.com

3.2.48. $\underline{\mathrm{APC}}$

Table 3.2.48. Data for the Effect of the Compounds on APC Activity

\begin{tabular}{|c|c|c|c|c|c|c|c|}
\hline \multirow[t]{2}{*}{ Compounds } & \multicolumn{2}{|c|}{$\begin{array}{c}\text { APC Activity } \\
\text { (Fluorescence count) }\end{array}$} & \multicolumn{2}{|c|}{$\begin{array}{c}\text { Background } \\
\text { (Fluorescence count) }\end{array}$} & \multicolumn{2}{|c|}{$\%$ Activity } & \multirow[t]{2}{*}{$\%$ Inhibition } \\
\hline & Repeat1 & Repeat2 & Repeat1 & Repeat2 & Repeat1 & Repeat2 & \\
\hline No Compound & 2659 & 2530 & 215 & 190 & 103 & 97 & 0 \\
\hline NCGC00167526, $10 \mu \mathrm{M}$ & 981 & 976 & 144 & 136 & 23 & 23 & 77 \\
\hline NCGC00160398, $10 \mu \mathrm{M}$ & 9268 & 9322 & 8853 & 8905 & 1 & 4 & 98 \\
\hline NCGC00025297, $10 \mu \mathrm{M}$ & 498 & 532 & 126 & 137 & 0 & 2 & 99 \\
\hline Dabigatran, $0.2 \mu \mathrm{M}$ & 2243 & 2216 & 136 & 175 & 85 & 84 & 16 \\
\hline Dabigatran, $2 \mu \mathrm{M}$ & 1676 & 1711 & 195 & 157 & 56 & 58 & 43 \\
\hline Dabigatran, $20 \mu \mathrm{M}$ & 615 & 598 & 133 & 131 & 6 & 5 & 95 \\
\hline Background & 550 & 559 & 190 & 184 & & & \\
\hline
\end{tabular}

\section{APC Activity}

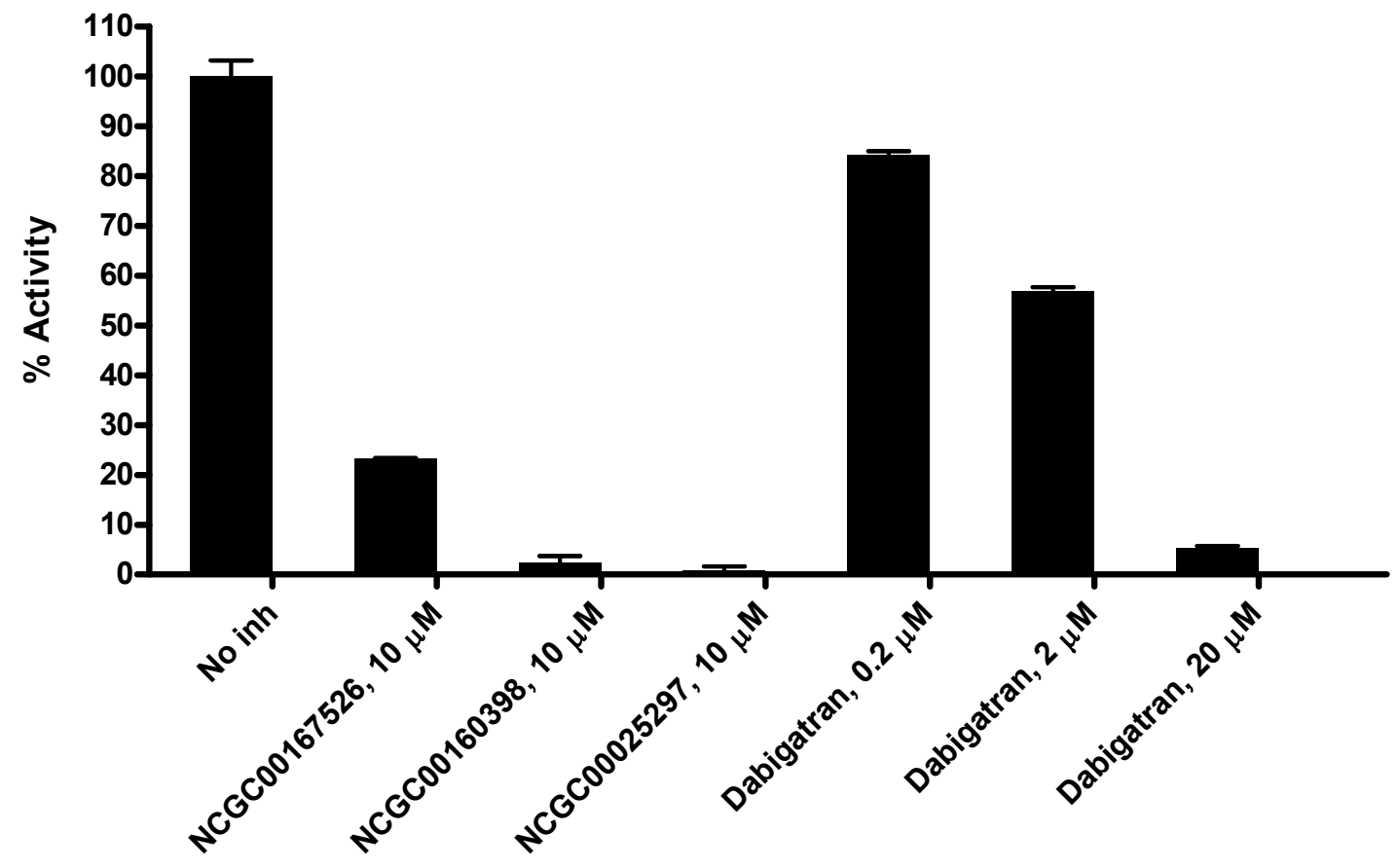


6042 Cornerstone Court West, Suite B

San Diego, CA 92121

Tel: 1.858 .829 .3082

Fax: 1.858 .481 .8694

Email: info@bpsbioscience.com

\section{Quality Assurance Statement}

I certify that the results presented in this report were generated using the materials and methods mentioned and that these results reflect the Raw Data.

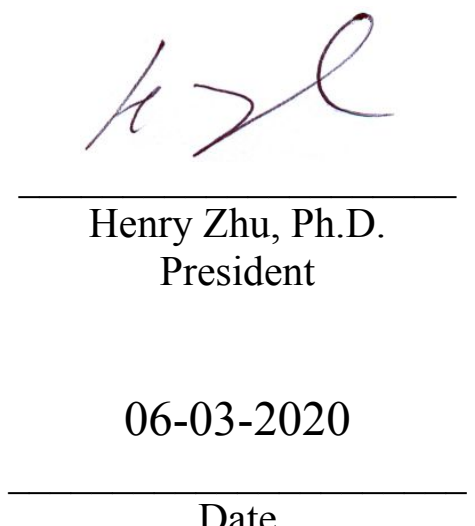

Date 